\title{
Chapter 10 \\ The Largest Accelerators and Colliders of Their Time
}

\author{
K. Hübner, S. Ivanov, R. Steerenberg, T. Roser, J. Seeman, K. Oide, \\ Karl Hubert Mess, Peter Schmüser, R. Bailey, and J. Wenninger
}

\subsection{Proton Accelerators and Colliders}

\author{
K. Hübner $\cdot$ S. Ivanov $\cdot$ R. Steerenberg
}

\subsubsection{CERN Proton Synchrotron (CPS)}

The Study Group for a GeV-scale Proton Synchrotron was launched in 1952 at CERN. Initially, an up-scaled version of the $3 \mathrm{GeV}$ Cosmotron was considered but soon a new design based on the newly discovered alternating-gradient principle and promising a proton energy of $30 \mathrm{GeV}$ was adopted by the CERN Council in the same year. In order to limit cost the energy was subsequently limited to $25 \mathrm{GeV}$

\footnotetext{
K. Hübner $\cdot$ R. Steerenberg $\cdot$ K. Oide $\cdot$ K. H. Mess $\cdot$ R. Bailey $(\varangle) \cdot$ J. Wenninger CERN (European Organization for Nuclear Research) Meyrin, Genève, Switzerland e-mail: Rende.Steerenberg@cern.ch; roger.bailey@cern.ch; jorg.wenninger@cern.ch

S. Ivanov

Institute of High Energy Physics, Moscow, Russia

e-mail: sergey.ivanov@ihep.ru

T. Roser

Brookhaven National Laboratory, New York, NY, USA

e-mail: roser@bnl.gov

J. Seeman

SLAC National Accelerator Laboratory, Stanford University, Menlo Park, CA, USA

e-mail: seeman@slac.stanford.edu

P. Schmüser

DESY, Hamburg, Germany

e-mail: Peter.Schmueser@desy.de

(C) The Author(s) 2020

S. Myers, H. Schopper (eds.), Particle Physics Reference Library, https://doi.org/10.1007/978-3-030-34245-6_10
} 
Table 10.1 Basic parameters of the CPS [5]

\begin{tabular}{l|l}
\hline Accelerated particles & Protons, lead ions \\
\hline Momentum protons/lead ions & $26 \mathrm{GeV} / \mathrm{c}, 5.9 \mathrm{GeV} / \mathrm{c}$ nucleon \\
\hline Circumference $[\mathrm{m}]$ & $200 \pi$ \\
\hline Magnetic lattice & Alternating-gradient focusing, combined-function \\
\hline Focusing order & FOFDOD \\
\hline Magnetic field index & $n=288$ \\
\hline Number of main magnets & 100 \\
\hline Bending magnetic field & $0.1013 \mathrm{~T}$ (inj. at $1 \mathrm{GeV}), 1.25 \mathrm{~T}($ extr. at $26 \mathrm{GeV} / \mathrm{c})$ \\
\hline Betatron oscillations/turn & $6.24(\mathrm{~h}), 6.26(\mathrm{v})$ \\
\hline Rise time/flat top time & $0.7 \mathrm{~s} / 0.3 \mathrm{~s}(26 \mathrm{GeV} / \mathrm{c})$ \\
\hline Long straight sections & Number $=20$, length $=3.0 \mathrm{~m}$ \\
\hline RF system (tunable) & 11 cavities, $2.8-9.55 \mathrm{MHz}, 220 \mathrm{keV} /$ turn total maximum \\
\hline Auxiliary RF systems & $13,20,40,80,200 \mathrm{MHz}$ \\
\hline Vacuum chamber & Stainless steel, $146 \times 70 \mathrm{~mm}{ }^{2}$ in the bending magnets \\
\hline
\end{tabular}

and the project led by J.B. Adams was approved in 1953. The final parameters were fixed in 1954 and construction started in 1955. The CPS [1] became operational towards the end of 1959 reaching an energy of $28 \mathrm{GeV}$ [2, 3]. It has turned out to be an extremely versatile facility [4] (Table 10.1).

Initially, the proton injector was a $50 \mathrm{MeV}$ Alvarez-type linear accelerator (linac L1) operating at $200 \mathrm{MHz}$. In order to increase the intensity a four-ring synchrotron booster (PSB) was inserted in 1972 between L1 and CPS raising the kinetic injection energy to $0.8 \mathrm{GeV}$. Over the years, it had its top kinetic energy raised in steps to $1 \mathrm{GeV}$ in 1985 and $1.4 \mathrm{GeV}$ in 1999 to allow for the production of the LHC beams. As part of the LHC Injectors Upgrade (LIU) project [6] a further increase to $2 \mathrm{GeV}$ is being prepared at present for first beam in 2020. In 1979, L1 was replaced by linac 2 (L2) of a more modern and robust design. The $50 \mathrm{MeV}$ proton linac 2 is presently being replaced by the new linac 4 (L4) [7] that will provide $\mathrm{H}^{-}$ions at $160 \mathrm{MeV}$ to the charge exchange injection equipped PS Booster.

The CPS provided initially secondary beams by means of internal targets. Since 1967 a fast extraction system over one turn became available for fixed-target physics complemented in 1969 by a system providing slow-extracted beams spilling out particles over a large number of turns. The fast extraction was used to produce neutrino beams with protons and, between 1970 and 1983, $26 \mathrm{GeV} / \mathrm{c}$ protons for the Intersecting Storage Rings (ISR). At present, the fast extraction provides a $26 \mathrm{GeV} / \mathrm{c}$ proton beam $\left(1.5 \times 10^{13}\right.$ per pulse) for the Antiproton Decelerator (AD) after compression of the four equidistant bunches that occupy half the CPS circumference. This manipulation by the radio-frequency accelerating system makes this proton bunch train so short that the secondary antiproton bunch train fits into the AD circumference being one quarter of that of the CPS. Further, a $20 \mathrm{GeV} / \mathrm{c}$ single high-intensity bunch containing up to $9 \times 10^{12}$ protons is extracted after a non-adiabatic bunch shortening to produce neutrons from a lead-target. Since 2010, a large variety of LHC beams have been produced that over time have become much 
Table 10.2 Pre- and post-LIU main LHC beam parameters at $26 \mathrm{GeV} / \mathrm{c}$ PS extraction

\begin{tabular}{l|l|l|l|l}
\hline & Beam type & $N\left[\times 10^{11} \mathrm{p}\right]$ & $\varepsilon[\mathrm{mm} \mathrm{mrad}]$ & $N_{b} /$ xtr \\
\hline \multirow{2}{*}{2018} & Standard & 1.4 & 2.3 & 72 \\
\cline { 2 - 5 } & BCMS & 1.3 & 1.2 & 48 \\
\hline \multirow{2}{*}{ Post-LIU } & Standard & 2.6 & 1.85 & 72 \\
\cline { 2 - 5 } & BCMS & 2.6 & 1.45 & 48 \\
\hline
\end{tabular}

brighter. As foreseen in the LHC design report [8] the PS produces single bunch beams varying in intensity from $0.05 \times 10^{11}$ to $1.2 \times 10^{11}$ protons. For the initial multi-bunch beams $12-72$ bunches per extraction at $26 \mathrm{GeV} / \mathrm{c}$ with respective bunch spacings of $25,50,75$ and $150 \mathrm{~ns}$ were routinely produced. The highest LHC bunch intensity of $1.7 \times 10^{11}$ protons per bunch in a transverse emittance of $1.6 \mathrm{~mm} \mathrm{mrad}$ and a bunch train length of 36 bunches was reached with the $50 \mathrm{~ns}$ bunch spacing that was initially used to limit electron cloud effects in the LHC. By mid-July 2015 the $\mathrm{LHC}$ requested the $25 \mathrm{~ns}$ bunch spacing with $1.15 \times 10^{11}$ protons per bunch in a transverse emittance of $2.5 \mathrm{~mm}$ mrad and 72 bunches per extraction. Thanks to the versatile PS RF system an even brighter LHC beam, based on Bunch Merging, Compression and Splitting (BCMS), was established. Up to $1.3 \times 10^{11}$ protons per bunch in a transverse emittance of $1.1 \mathrm{~mm}$ mrad and 48 bunches per extraction are delivered to the SPS since mid-2016. The LIU project aims at increasing the bunch intensity to $2.6 \times 10^{11}$ protons per bunch for both beam types as given in Table 10.2.

For fixed-target experiments at the Super Proton Synchrotron (SPS), a $14 \mathrm{GeV} / \mathrm{c}$ proton beam $\left(3 \times 10^{13}\right.$ per pulse) was spilled out over five turns by cutting it with an electro-static septum in horizontal phase space in order to fill by box-car stacking 5/11 parts of the SPS circumference being 11 times longer than the one of the CPS. A second CPS beam pulse fills further $5 / 11$ parts, thus leaving $1 / 11$ for the kicker rise-times. Since 2010 a novel scheme, using a fourth order betatron resonance for capturing the beam in five stable islands in the horizontal phase space, avoids the losses at the electrostatic extraction septum [9].

The slow-extraction based on a third-order betatron resonance is still in use to produce primary $24 \mathrm{GeV} / \mathrm{c}$ proton beams. Up to $5 \times 10^{11}$ protons can be spilled out in up to $450 \mathrm{~ms}$ for the production of secondary beams for fixed-target experiments at the CPS, but also for primary protons to the IRRAD and CHARM irradiation facilities [10].

As soon as L2 was available, L1 was modified to provide Deuterium and $\alpha$ particle beams for collisions in the ISR and, later, Sulphur beams for fixed target experiments in the SPS. In 1994, L1 was replaced by linac 3 (L3) providing 4.2 MeV/amu $\mathrm{Pb}^{+53}$ ions which, fully stripped after CPS extraction, are used for fixed-target experiments in the SPS. Since 2010 the lead ions are also fast-ejected to the SPS for lead-lead collisions in LHC. They no longer pass through the PSB but a small storage ring, the Low Energy Ion Ring (LEIR), acts as accumulator between the fast-cycling L3 and the slow-cycling CPS and is equipped with stochastic and 
electron cooling to decrease the transverse emittance of the accumulated beam. The CPS provides a total of $8 \times 10^{10}$ ions per pulse in four bunches.

The acceleration cycles for the different users are grouped in a supercycle depending on the user requirements allowing for a quick, reproducible switching from one to another mode of operation [11].

\subsubsection{Brookhaven Alternating Gradient Synchrotron (AGS)}

After successful completion of the $3 \mathrm{GeV}$ Cosmotron in 1952 the design study for a more powerful accelerator was launched coincident with the invention of the alternating-gradient principle [12, 13]. Construction led by G.K. Green and J.P. Blewett started in 1953 and commissioning was completed with the first proton beam accelerated to $31 \mathrm{GeV}$ in July 1960 [14]. In order to test experimentally whether the beam would pass transition energy, an electron analogue had been built in 1954 and operated until 1957. This model had a circumference of $43.1 \mathrm{~m}$ accelerating electrons from 1 to $10 \mathrm{MeV}$ with transition energy at $3.5 \mathrm{MeV}$. In order to reduce cost, the alternating-gradient, strong-focusing was provided by electrostatic lenses and bending by electrostatic fields [15]. The test showed that transition can be crossed without problems but the price was a delay in the AGS construction relative to the CERN PS, which however was at the end compensated by better preparation of the experimental programme compared to CERN.

The first injector was a $50 \mathrm{MeV}$ Alvarez-type proton linear accelerator. The present $200 \mathrm{MeV}$ linear accelerator began operation in 1970 . In $1982 \mathrm{H}^{-}$charge exchange injection into the AGS was introduced [16] and in 1991 a $1.5 \mathrm{GeV}$ booster synchrotron was commissioned [17]. The Booster can provide $1.5 \times 10^{13}$ protons per pulse at $1.9 \mathrm{GeV}$ at the design repetition frequency of $7.5 \mathrm{~Hz}$. The acceleration harmonic schemes (Booster harmonic, AGS harmonic, transfers) evolved from (3, $4,12)$ to $(2,4,8)$ and finally to $(1,6)$ in pursuit of higher intensity [18] (Table 10.3).

Secondary beams from the AGS were initially provided from internal targets. This also creates high beam loss and activation in the accelerator not compatible with high-intensity operation. The first fast-extraction was installed in the mid-60s followed by slow-extraction in 1967 which served up to six target stations and spilling out protons with repetition periods from $1.8 \mathrm{~s}$ to $5.8 \mathrm{~s}$. To cope with the intensity increases, the AGS underwent a series of upgrades including a new main magnet power supply, addition of transverse feed-back, special magnets to provide fast crossing of the transition energy, and a high power RF system [20]. In the early 2000s the AGS provided a slow-extracted beam of $7 \times 10^{13}$ protons per pulse at $24 \mathrm{GeV}[21]$.

With the appropriate source added to the $200 \mathrm{MeV}$ linear accelerator, polarized protons have been produced by the injector chains from 1985 onward for fixed target experiments. To meet injector requirements for RHIC - intensity and polarizationthe polarized source underwent a major upgrade [22]. The polarization transmission efficiency in the AGS has been substantially improved with the installation of two 
Table 10.3 Basic parameters of the AGS [19]

\begin{tabular}{l|l}
\hline Accelerated particles & Protons, polarized protons, heavy ions (up to $\mathrm{Au}$ ) \\
\hline Particle energy & $30 \mathrm{GeV}, 25 \mathrm{GeV}, 14.5 \mathrm{GeV} / \mathrm{n}$ \\
\hline Circumference $[\mathrm{m}]$ & $256.9 \pi$ \\
\hline Magnetic lattice & Alternating-gradient focusing, combined-function \\
\hline Focusing order & $(\mathrm{F} / 2) \mathrm{O}(\mathrm{F} / 2)(\mathrm{D} / 2) \mathrm{O}(\mathrm{D} / 2)$ \\
\hline Magnetic field index & $n=365$ \\
\hline Number of main magnets & 240 \\
\hline Bending magnetic field & $0.105 \mathrm{~T}$ at injection, $1.31 \mathrm{~T}$ at maximum particle momentum \\
\hline Betatron oscillations/turn & $8.75(\mathrm{~h}), 8.75(\mathrm{v})$ \\
\hline Rise time/flat top time & $0.6 \mathrm{~s} / 0.5$ to $2.5 \mathrm{~s}$ \\
\hline Long straight sections & Number $=24$, length $=3.15 \mathrm{~m}$ \\
\hline RF system (tunable) & 10 cavities, 1.8 to $4.5 \mathrm{MHz}, 200 \mathrm{KeV} /$ turn total maximum \\
\hline Auxiliary RF system (fixed RF) & $92 \mathrm{MHz}$ \\
\hline Vacuum chamber & Inconel, $173 \times 78 \mathrm{~mm}{ }^{2}$ in the bending magnets \\
\hline
\end{tabular}

"partial Siberian" snakes $[23,24]$ and a system to rapidly cross weak resonances. Polarization at transfer to RHIC ( $24 \mathrm{GeV}$ ) is $70 \%$ (with $82 \%$ at $200 \mathrm{MeV}$ ) and with intensity $2 \times 10^{11}$ protons per bunch [25, 26].

Since 1986 the Booster has also accelerated ions (d to Au) using a Tandem Van de Graaff as injector. For RHIC operation about $5 \times 10^{9} \mathrm{Au}^{31+}$ ions at $41.6 \mathrm{MeV} / \mathrm{n}$ are injected over 60 turns into the Booster, accelerated to $101 \mathrm{MeV} / \mathrm{n}$, stripped to $\mathrm{Au}^{77+}$ and injected into the AGS. The ions are fully stripped before injection into RHIC [27]. A new pre-injector is being commissioned based on an EBIS source followed by a new linear accelerator [28]. The new system increases the available ions for RHIC to include Uranium [29].

\subsubsection{The 70 GeV Proton Synchrotron (U-70) of NRC "Kurchatov Institute": IHEP (Protvino)}

The study of a powerful synchrotron started in the mid-60s in the then Soviet Union and focused onto the Protvino site since 1958 [30]. The project was led by V.V. Vladimirski from 1958 and A.A. Logunov from 1963 after the foundation of the Institute for High-Energy Physics. The construction started in 1961 and the commissioning [31] took place in 1967 culminating in a test run at $76 \mathrm{GeV}$ in the same year, which was the world record at that time (Table 10.4).

The first proton injector has been the $100 \mathrm{MeV}$ Alvarez-type DTL proton linear accelerator (I-100) providing a pulse current of $100 \mathrm{~mA}$ over five turns in U-70. It served until 1985 as injector [34] and is still in operation as light ion injector [33]. In order to increase the intensity, the $1.5 \mathrm{GeV}$ booster synchrotron (U-1.5) cycling at $16^{2} / 3 \mathrm{~Hz}$ came on line in 1985 [35]. Its injector is a $30 \mathrm{MeV}$ RFQ-type DTL linear 
Table 10.4 Basic parameters of the U-70 [32, 33]

\begin{tabular}{l|l}
\hline Accelerated particles & Protons, carbon nuclei \\
\hline Particle energy & $70 \mathrm{GeV}(34 \mathrm{GeV} / \mathrm{n}$ carbon nuclei) \\
\hline Circumference & $1483.7 \mathrm{~m}$ \\
\hline Magnetic lattice & Alternating-gradient focusing, combined-function \\
\hline Focusing order & FODO \\
\hline Magnetic field index & $n=443$ \\
\hline Number of main magnets & 120 \\
\hline Bending magnetic field & $0.035 \mathrm{~T}$ at injection, $1.2 \mathrm{~T}$ at maximum energy \\
\hline Betatron oscillations/turn & $9.9(\mathrm{~h}), 9.8(\mathrm{v})$ \\
\hline Rise time/flat top time & $2.8 \mathrm{~s} / 2 \mathrm{~s}$ \\
\hline Long straight sections & Number $=24$, length $=4.87 \mathrm{~m}$ \\
\hline RF system (tunable) & $38(+2$ spare $)$ cavities, $2.6-6.1 \mathrm{MHz}, 150 \mathrm{keV} /$ turn total \\
& maximum \\
\hline Auxiliary RF system (fixed RF) & 2 cavities at $200 \mathrm{MHz}, 500 \mathrm{kV}$ peak total voltage \\
\hline Vacuum chamber & stainless steel, $200 \times 100 \mathrm{~mm}{ }^{2}$ in the bending magnets \\
\hline
\end{tabular}

accelerator (URAL-30) injecting 1-4 turns into the booster with a pulse current up to $80 \mathrm{~mA}$. Twenty nine single-bunch pulses of the booster with up to $8 \times 10^{11}$ protons per pulse build up the beam in U-70 within $1.8 \mathrm{~s}$ using bunch-to-bucket transfer. The U-1.5 has a circumference $1 / 15$ times the one of U-70. It operates now at $1.3 \mathrm{GeV}$ limited by the power supply.

The U-70 accelerator has been equipped with a new vacuum chamber in 1997 and the control system has been modernized from 1998 onwards. Thanks to all these upgrades and, in particular, to the addition of the booster and the new linear accelerator, the beam intensity has reached $1.5 \times 10^{13}$ protons per pulse with a repetition time of $9.8 \mathrm{~s}$, which has to be compared with the initially planned $1 \times 10^{12}$ protons per pulse [32]. In recent years, U-70 operates with $1.1 \times 10^{13}$ protons per pulse at $50 \mathrm{GeV}$ to save energy [36].

Initially, only internal targets have provided a large variety of secondary beams and some internal targets are still in use providing spills up to $1.8 \mathrm{~s}$. A fast-extraction system has been added soon. A slow-extraction system based on a third order resonance came into operation in 1979 and it has been upgraded for higher intensity in 1989. Its spill-length could be extended up to $1.3 \mathrm{~s}$. Now, a stochastic slow extraction system provides smooth spills over up to $3 \mathrm{~s}$ at top energy. Extraction, beam splitting and collimation using bent crystals have been achieved being under study since 1990 [37].

Deuterons have been accelerated from 2008 onwards in I-100 and U-1.5 to 16.7 MeV/n and 455 MeV/n, respectively. In 2009 deuterons were further accelerated in U-70 to $23.6 \mathrm{GeV} / \mathrm{n}$ corresponding to $50 \mathrm{GeV}$ protons. The latter has the potential to accelerate them to $34 \mathrm{GeV} / \mathrm{n}$. An intensity of $5 \times 10^{10}$ deuterons per pulse has been achieved.

In 2011, carbon ions have been first accelerated in I-100, U-1.5 and U-70 to $34 \mathrm{GeV} / \mathrm{n}$ [38]. In 2013, validation tests of all the top-energy extractions available 
with carbon beam have been accomplished successfully. Carbon beam intensity is $3-5 \times 10^{9}$ ions per pulse $(8.2 \mathrm{~s})$, in a single bunch. At the DC flat-bottom, U-70 now also operates in a beam storage- and stretcher-ring mode for $455 \mathrm{MeV} / \mathrm{n}$ carbon ions enabling their square-wave slow stochastic extraction $(0.5-1 \mathrm{~s}$ long) via a PiccioniWright technique for an applied fixed-target research $[39,40]$.

\subsubsection{The CERN Intersecting Storage Rings (ISR)}

The first ideas for a realistic proton-proton collider were publicly discussed in 1956 [41, 42]. The Accelerator Research Group set up by the CERN Council in 1956 formulated in 1960 the first proposal for a proton-proton collider attached to the CERN PS. In 1960 construction began on a small-proof-of-principle $1.9 \mathrm{MeV}$ electron storage ring, the CERN Electron Storage and Accumulation Ring (CESAR) which experimentally proved the accumulation of particles by RF stacking in 1964, an essential technique [43] to build up intense beams, a prerequisite for getting the required luminosity. CESAR also was an important test bed for the for Ultra-High Vacuum (UHV) technology which had to developed to achieve a very low vacuum pressure, indispensable for a long lifetime of stored beams. The Design report [44] was issued in 1964, the project was approved in 1965, and construction lead by K. Johnsen started without delay. The first proton-proton collisions took place in 1971 with a beam momentum up to $26.5 \mathrm{GeV} / \mathrm{c}$, the maximum momentum available from the CPS. The ISR consisted of two independent storage rings intersecting at eight points at an angle of $14.8^{\circ}$. To create space for long straight sections in the interaction regions, the circumference of the ring was 1.5 times of that of the CPS which supplied particles to the ISR through two long transfer lines. The ISR operated for physics as collider from 1971 to 1983 . It was decommissioned after 1984 (Table 10.5).

The CPS was the injector for the ISR supplying mainly protons up to $26.5 \mathrm{GeV} / \mathrm{c}$. Typical intensities were $3 \times 10^{12}$ protons per pulse in 20 bunches every $2.4 \mathrm{~s}$ during the filling process. The filling of one ISR ring took less than $10 \mathrm{~min}$. Later, also deuterons, alpha particles and antiprotons were accelerated for the ISR.

The ISR team had to tackle a number of technological challenges but the most important was to assure UHV imperative for a long beam lifetime. The stainless steel vacuum chamber was in situ bakeable eventually to $300{ }^{\circ} \mathrm{C}$. Pumping was provided by sputter ion-pumps and, at critical places, Ti-sublimation pumps. All vacuum chambers had to be glow-discharge cleaned. The continuous effort eventually resulted in average pressure below $10^{-11}$ Torr ( $\mathrm{N}_{2}$ equivalent) reducing beam loss rates to typically around one part per million per minute during physics runs. Beams of physics quality could last 40-50 h. Beam currents of 10 A were achieved already after start-up. Later up to $57 \mathrm{~A}$ were stored per ring with 30-40 A as typical value. The proton-proton initial luminosity (design $4 \times 10^{30} \mathrm{~cm}^{-2} \mathrm{~s}^{-1}$ ) was increased from $1.6 \times 10^{30} \mathrm{~cm}^{-2} \mathrm{~s}^{-1}$ in 1971 to $1.4 \times 10^{32} \mathrm{~cm}^{-2} \mathrm{~s}^{-1}$ in the superconducting low-beta section installed in one of the interaction points in 1982, which stayed the 
Table 10.5 Basic parameters of the ISR [45]

\begin{tabular}{l|l}
\hline Colliding particles & $\mathrm{pp}, \mathrm{dd}, \mathrm{pd}, \alpha \alpha, \alpha \mathrm{p}, \mathrm{p} \overline{\mathrm{p}}$ \\
\hline Particle momentum & $3.5-31.4 \mathrm{GeV} / \mathrm{c}$, typically $26 \mathrm{GeV} / \mathrm{c}$ \\
\hline Circumference $[\mathrm{m}]$ & $300 \pi$ \\
\hline Magnetic lattice & Alternating-gradient focusing, combined-function \\
\hline Focusing order & FODO \\
\hline Number of main magnets & 132 per ring \\
\hline Magnetic dipole field & $1.33 \mathrm{~T}$ at maximum momentum \\
\hline Length of main magnets & $4.88 / 2.44 \mathrm{~m}$ \\
\hline Magnetic field index & $n=248$ \\
\hline Betatron oscillations/turn & $8.90(\mathrm{~h}), 8.88(\mathrm{v})$ \\
\hline Long straight sections & Number $=8$, length $=16.8 \mathrm{~m}$ \\
\hline$\beta_{*}(\mathrm{~h} / \mathrm{v})$ & $21 \mathrm{~m} / 12 \mathrm{~m}$ \\
\hline$\beta_{*}(\mathrm{~h} / \mathrm{v})$ & $2.5 \mathrm{~m} / 0.28 \mathrm{~m}$ in superconducting low-beta section \\
\hline RF system per ring & 7 cavities, $9.5 \mathrm{MHz}, 16 \mathrm{kV} \mathrm{RF} \mathrm{peak} \mathrm{voltage}$ \\
\hline Auxiliary RF system & $3 \mathrm{rd}$ harmonic \\
\hline Vacuum chamber & Stainless steel, $160 \mathrm{~mm} / 52 \mathrm{~mm}$ full width $(\mathrm{h} / \mathrm{v})$ \\
\hline
\end{tabular}

world record luminosity until 1991 . The superconducting low beta-section had been preceded by an insertion based on conventional magnets, operational from 1974. From 1973 onwards, the beams could be accelerated in the ISR to $31.4 \mathrm{GeV} / \mathrm{c}$ by phase displacement acceleration [46].

From 1976 onward deuterons were stored in the ISR so that dd and pd collisions became available. Alpha particles were stored in 1980 for $\alpha \alpha$ and $\alpha$ p collisions. Initial dd luminosities reached $1.6 \times 10^{30} \mathrm{~cm}^{-2} \mathrm{~s}^{-1}$ and were $4 \times 10^{28} \mathrm{~cm}^{-2} \mathrm{~s}^{-1}$ in the $\alpha \alpha$ case. Antiprotons were stored as soon as the antiproton injector complex had become operational in 1981 (see Sect. 10.1.6). This required a new transfer line from the CPS.

The ISR will be remembered for a number of breakthroughs in accelerator physic and technology: UHV-technology for a large scale facility, control of intense coasting beams, discovery of Schottky scans, experimental demonstration of stochastic cooling, and absolute luminosity measurement by van der Meer scans [46].

\subsubsection{The CERN Super Proton Synchrotron (SPS)}

Design studies of a powerful proton synchrotron started at CERN in 1961 when the Accelerator Research Division had been created, not long after the CPS had become operational in 1960 . The design energy was $300 \mathrm{GeV}$ and the specified flux $10^{13}$ protons/s. A study group lead by K. Johnsen presented a first design report in 1964 [47]. However, the idea that the facility should be constructed on a green field, i.e. not in Switzerland near CERN, made the choice of the site difficult and funding 
Table 10.6 Basic parameter of the SPS in proton fixed-target mode [51]

\begin{tabular}{l|l}
\hline Accelerated particles & Protons \\
\hline Momentum protons & $450 \mathrm{GeV} / \mathrm{c}$ \\
\hline Circumference $[\mathrm{m}]$ & $2200 \pi$ \\
\hline Magnetic lattice & Alternating-gradient focusing, separated-function \\
\hline Focusing order & FODO \\
\hline Number of dipole magnets & 744 \\
\hline Dipole magnetic field & $0.056 \mathrm{~T}$ at injection, $1.8 \mathrm{~T}$ at maximum particle momentum \\
\hline Betatron oscillations/turn & $26.6(\mathrm{~h}), 26.6(\mathrm{v})$ \\
\hline Rise time/flat top time & $0.75 \mathrm{~s} / 2.5 \mathrm{~s}$ \\
\hline Long straight sections & Number $=6$, length $=128 \mathrm{~m}$ \\
\hline RF system & 4 traveling-wave structures at $200 \mathrm{MHz}, 4 \mathrm{MeV} /$ turn total \\
\hline Vacuum chamber & Stainless steel, $150 \times 50 \mathrm{~mm}^{2}$ \\
\hline
\end{tabular}

problems delayed the decision over many years. In 1969, J.B. Adams was appointed leader of the $300 \mathrm{GeV}$ Programme and the project gathered new momentum with the suggestion to construct the facility close to the existing laboratory and using the CPS as injector, which had been advocated already in 1961 by C. Ramm [48]. This and a new design based on the alternating-gradient principle but with the function of bending and focusing separated instead of combined-function allowed a considerable cost reduction [49]. The project was approved in 1971 and a beam energy of $400 \mathrm{GeV}$, exceeding the design energy, was reached in 1976 after almost a decade of planning and decision making [50] (Table 10.6).

The CPS acts as proton injector having 1/11th circumference of the SPS. Initially, the SPS beam was created by peeling off the required beam from the CPS beam by an electrostatic septum over 10 turns of the CPS at $10 \mathrm{GeV} / \mathrm{c}$, leaving $1 / 11$ th of the circumference for the SPS injection kicker fall-time and ejection kicker rise-time, in a process called continuous transfer. From 1978 onwards, after the CPS intensity had been increased by the new linac 2 and the booster synchrotron, two CPS pulses were consecutively sent to the SPS, each CPS pulse was peeled five times. The CPS acted also as positron-electron injector during lepton operation for LEP and as injector during the fixed-target ion runs. It provides also a $26 \mathrm{GeV} / \mathrm{c}$ proton beam and $5.9 \mathrm{GeV} / \mathrm{u}$ ion beams to the SPS when the latter is used to fill the CERN Large Hadron Collider (LHC).

The intensity of the proton beam for fixed-target experiments had been raised gradually. In 1984, the injection momentum was raised to $14 \mathrm{GeV} / \mathrm{c}$ providing a more stable, reproducible injection and a beam of lower emittance. After the kinetic beam energy of the PS-Booster (PSB) had been raised to $1.4 \mathrm{GeV}$ in 1998 resulting in a further emittance reduction, the SPS delivered more than $4 \times 10^{13}$ protons per pulse with a record value of $5.3 \times 10^{13} \mathrm{ppp}$.

Since the start-up in 1976 three types of extraction modes have been available towards the two experimental areas: (i) fast extraction of part or the entire beam (spill 3-23 $\mu \mathrm{s}$ ); (ii) slow-resonant extraction (0.5-2 s); (iii) fast-resonant extraction $(<3 \mathrm{~ms})$. A typical pattern was extraction to the West-Area at an energy of $200 \mathrm{GeV}$ 
( $250 \mathrm{GeV}$ maximum) during a short pause in the acceleration followed by extraction at top energy to the North Hall. After the upgrading to $450 \mathrm{GeV}$ of the transfer line to the West Hall in 1983, simultaneous resonant extraction to both areas was implemented by an appropriate adjustment of the horizontal betatron phase advance in the ring. The sharing ratio between the two clients was fully adjustable. Extraction towards the West Hall was terminated in 2003 to free resources for LHC.

In 1986 and 1987 two exploratory fixed-target runs with ions took place after linac 2 had become operational freeing linac 1 which had in the meantime been equipped with the appropriate front-end for ion operation. In 1986, fully stripped oxygen ions were accelerated for the first time to $200 \mathrm{GeV} / \mathrm{n}$ after the SPS had been set up with a deuteron beam of more convenient higher intensity. In the second run, in 1987, sulphur ions were used. The sulphur runs were resumed from 1990 to 1992 providing $9 \times 10^{9}$ charges per pulse with four batches injected from the CPS. After the construction of the dedicated heavy-ion linac (linac 3) lead ions at $177 \mathrm{GeV} / \mathrm{u}$ were available for the experiments from 1994 to 2002 and indium at $158 \mathrm{GeV} / \mathrm{u}$ in 2003. No ion runs took place in 1997 and 2001. The SPS delivered up to $6 \times 10^{10}$ fully-stripped ions in terms of charges per pulse [52].

It is worthwhile to mention that the SPS also accelerated electrons and positrons for injection into LEP in the years 1989 to 2000. This had required substantial modifications. Since the traveling-wave cavities could accelerate the beam only to $14 \mathrm{GeV}, 32$ copper-based standing-wave cavities operating at $200 \mathrm{MHz}$ and providing a peak-voltage of $30 \mathrm{MV}$ were added. Ejection and injection channels were equipped appropriately and a campaign of meticulously shielding the magnet coils against synchrotron radiation was conducted [53]. The LEP injection energy was first set to $20 \mathrm{GeV}$ and, later, when the standing-wave cavities were replaced by two four-cell superconducting RF structures, the LEP injection energy could be raised to $22 \mathrm{GeV}$.

The SPS acts as injector into the LHC providing protons at $450 \mathrm{GeV}$ since 2008 and ions at $176 \mathrm{GeV} / \mathrm{u}$ since 2010 [54]. Lead is the preferred ion species for the first years of operation. This new role required a number of hardware modifications and, in particular, the addition of a new extraction system for the anti-clockwise LHC ring which serves also the target for the new long-base line neutrino beam towards Gran Sasso in Italy. Two new beam lines towards the LHC had to be built.

\subsubsection{The CERN Super Proton Synchrotron (SPS) as Proton-Antiproton Collider}

The proposal to use the SPS as proton-antiproton collider was made in 1976 [55]. The required increase of phase space density of the secondary antiprotons was to be produced by stochastic cooling invented by S. van der Meer [56] which had been experimentally demonstrated in the ISR [57]. Simultaneous stochastic cooling in transverse and longitudinal phase space at cooling rates several orders higher than 
Table 10.7 Basic parameter of the SPS in proton-antiproton collision mode [60]

\begin{tabular}{l|l}
\hline Accelerated particles & Protons and antiprotons \\
\hline Maximum particle energy & $315 \mathrm{GeV}$ \\
\hline Circumference $[\mathrm{m}]$ & $2200 \pi$ \\
\hline Magnetic lattice & Alternating-gradient focusing, separated-function \\
\hline Focusing order & FODO \\
\hline Number of dipole magnets & 744 \\
\hline Dipole magnetic field & $0.12 \mathrm{~T}$ at injection, $1.4 \mathrm{~T}$ at maximum particle energy \\
\hline Long straight sections & Number $=6$, length $=128 \mathrm{~m}$ (including 2 low- $\beta$ insertions) \\
\hline$\beta_{*}(\mathrm{~h} / \mathrm{v})$ & $1.0 \mathrm{~m} / 0.5 \mathrm{~m}$ \\
\hline Filling time & $29 \mathrm{~s}$ \\
\hline RF system & 4 traveling-wave structures at $200 \mathrm{MHz}, 3.6 \mathrm{MeV} /$ turn total \\
\hline Auxiliary RF system & $100 \mathrm{MHz}, 2 \mathrm{MV} /$ turn \\
\hline Vacuum chamber & Stainless steel, $150 \times 50 \mathrm{~mm}^{2}$ \\
\hline
\end{tabular}

those achieved in the ISR was proven to work in the Initial Cooling Experiment (ICE) in 1978. ICE was a small storage ring of $74 \mathrm{~m}$ circumference operating at 1.73 and $2.1 \mathrm{GeV} / \mathrm{c}$ and fed with protons from the CPS [58]. With the project decision taken in 1978, the construction of the Antiproton Accumulator Ring jointly led by R. Billinge and S. van der Meer started as well as the modifications of CPS and SPS. The first collision of protons and antiprotons occurred in 1981 and the data taking of the experiments took place from 1982 to 1991 except 1986 [59]. All modifications of the SPS for collider operation were removed after 1991 (Table 10.7).

The antiproton injector chain [59] consisted of the CPS with its proton injectors and of the Antiproton Accumulator storage ring (AA). The CPS produced an intense pulse of $1.2 \times 10^{13}$ protons at $26 \mathrm{GeV}$ every $4.8 \mathrm{~s}$ consisting of five bunches and having a pulse length matched to the circumference of the AA $(157 \mathrm{~m})$. The pulse impinged on a tungsten target followed by a magnetic horn or a lithium lens focusing the emerging antiprotons. The latter were collected in the AA operating at $3.5 \mathrm{GeV} / \mathrm{c}$, the momentum where the production was close to the peak. Subsequently, the stochastic cooling systems increased the phase space density by a factor of more than $5 \times 10^{8}$. The best daily production was close to $2 \times 10^{11}$ per day. For the SPS fill, the collected and cooled antiprotons were transferred to the CPS, accelerated to $26 \mathrm{GeV} / \mathrm{c}$ and injected into the SPS through a new transfer line built for anti-clockwise injection. The fill was terminated by acceleration of the protons in the CPS and their injection through the existing transfer line upgraded from $14 \mathrm{GeV} / \mathrm{c}$ to $26 \mathrm{GeV} / \mathrm{c}$. Subsequently, both beams were simultaneously accelerated to collision energy and, after the $\beta_{*}$ had been lowered, brought to collision by adjusting the separators. The duration of a coast was between 10 and $20 \mathrm{~h}$.

In order to increase the transverse and longitudinal acceptance after the target, an additional storage ring of $187 \mathrm{~m}$ circumference was constructed around the AA in 1986, the Antiproton Collector (AC). This new ring featured an RF system providing $1.5 \mathrm{MV}$ at $9.5 \mathrm{MHz}$ rotating the antiproton bunches in longitudinal phase space to reduce their momentum spread. It took over from AA the stochastic pre-cooling 
so that AA could be simplified but had its cooling systems upgraded. With these measures the antiproton production rate could be raised to more than $1 \times 10^{12}$ per day.

The SPS required a number of modifications: the vacuum ion pumps were doubled and Ti-sublimation pumps added resulting in a reduction of pressure from $2 \times 10^{-7}$ to $6 \times 10^{-9}$ Torr; an electrostatic deflector separated the beams horizontally at injection; the magnet lattice included two low-beta sections for focusing the beam in the two interaction points; the RF travelling wave structures at $200 \mathrm{MHz}$ had to accelerate particles travelling in both directions, and two underground experimental areas had to be constructed [61]. The initial beam energy was raised from 273 to $315 \mathrm{GeV}$ after an upgrade of the magnet cooling. Towards the end of the operation, the SPS was cycled between 100 and $450 \mathrm{GeV}$, thus providing collisions at $900 \mathrm{GeV}$ c.m. The performance of the collider was steadily increased during its lifetime by the upgrade of the antiproton injectors, in particular after the commissioning of AC; by adding electrostatic deflectors allowing to raise the number of bunches per beam from three to six avoiding collisions except in the two experiments and in the mid-arc between them; by the installation of a $100 \mathrm{MHz}$ RF system to increase the acceptance at injection. Hence, the average initial luminosity and the centre-of-mass energy rose from $0.05 \times 10^{30}$ at $546 \mathrm{GeV}$ in 1982 to $3 \times 10^{30} \mathrm{~cm}^{-2} \mathrm{~s}^{-1}$ at $630 \mathrm{GeV}$ with $\beta_{*}(\mathrm{~h} / \mathrm{v})$ at $0.6 \mathrm{~m} / 0.15 \mathrm{~m}$ in 1991 , the last year of collider operation. The collider collected an integrated luminosity of $17 \mathrm{pb}^{-1}$ during its lifetime [59].

\subsubsection{Acknowledgement}

Thanks to Karel Cornelis (CERN) for his critical reading and useful suggestions.

\subsubsection{Tevatron of Fermi National Laboratory (FNAL)}

The study of superconducting magnets started in 1972 in view of doubling the proton energy available at FNAL by adding another accelerator in the tunnel of the Main Ring (MR). In the same year, MR equipped with conventional magnets had reached its design energy producing $200 \mathrm{GeV}$ protons. Since a magnet study had shown the feasibility of ramped superconducting magnets of 4-5 T, the official design study of this new accelerator, the Tevatron, with the MR as injector was launched in 1974 under the leadership of R.R. Wilson. Project authorization was granted in 1979 and the accelerator reached $512 \mathrm{GeV}$ during commissioning in 1983 with the MR operating at $150 \mathrm{GeV}$ as injector [62]. The accelerator was then routinely used between 1983 and 2000 in eight runs for fixed-target physics in the three experimental areas dedicated to physics with mesons, neutrinos, and protons respectively. The beam energy was $800 \mathrm{GeV}$ except in the first run $(400 \mathrm{GeV})$ and 
Table 10.8 Basic parameters of the Tevatron [63]

\begin{tabular}{l|l}
\hline Accelerated/colliding particles & Protons/protons-antiprotons \\
\hline Particle energy & $800 \mathrm{GeV} / 980 \mathrm{GeV}$ \\
\hline Circumference $[\mathrm{m}]$ & $2000 \pi$ \\
\hline Magnetic lattice & Alternating-gradient focusing, separated-function \\
\hline Focusing order & FODO \\
\hline Number of main bending magnets & 774 \\
\hline Bending magnetic field & $0.67 \mathrm{~T}$ at injection, $4.35 \mathrm{~T}$ at maximum energy \\
\hline Rise time & $15 \mathrm{~s}$ \\
\hline Bending magnet & $\begin{array}{l}\text { Nb-Ti conductor at } 4.3 \mathrm{~K}, \text { cold-bore, iron at ambient } \\
\text { temperature }\end{array}$ \\
\hline Quadrupole field gradient & $11.4 \mathrm{~T} / \mathrm{m}$ at injection, $76 \mathrm{~T} / \mathrm{m}$ at maximum energy \\
\hline Betatron oscillations/turn & 20.59 (h), 20.59 $(\mathrm{v})$ \\
\hline Long straight sections & Number $=6$, length $=50 \mathrm{~m}$ (incl. 2 low- $\beta$ insertions) \\
\hline$\beta_{*}$ (h/v) & $0.28 \mathrm{~m}$ in low- $\beta$ sections \\
\hline RF system (tunable) & 8 cavities, $53.1 \mathrm{MHz}, 1.2 \mathrm{MeV} /$ turn total maximum \\
\hline Vacuum chamber & $\begin{array}{l}\text { Stainless steel, rounded square } 63 \mathrm{~mm} \text { full aperture (h,v) } \\
\text { (in dipoles) }\end{array}$ \\
\hline
\end{tabular}

the repetition rate of the order of one per minute. Slow-spills (20 s) and fast beam extraction ( $2 \mathrm{~ms}$ ) were available (Table 10.8).

The potential of the accelerator for proton-antiproton collisions with a centreof-mass energy close to $2 \mathrm{TeV}$ had been realized very early [64]. The bunched proton beam and anti-proton beam counter-rotating in the same vacuum chamber would be simultaneously accelerated in the Tevatron and brought to collision at top energy. Initiated in 1977, a study of the anti-proton beam cooling methods revealed that an anti-proton beam of sufficient intensity and density in phase space could be produced. This led to the construction of the anti-proton source in the period 1982-1985. The MR would also accelerate antiprotons and protons for injection into the Tevatron. It was equipped with two overpasses to provide space for the large detectors located in the two crossing points of the Tevatron. In 1985, the conversion of the Tevatron itself to a collider was started by the installation of the first low- $\beta$ section in the straight section housing later the CDF detector. First collisions were recorded at a centre-of-mass energy of $1.6 \mathrm{TeV}$ in 1985 and this energy could be raised to $1.8 \mathrm{TeV}$ in 1986 . It was increased again to $1.96 \mathrm{TeV}$ for Run II [65] which started in 2001 [66].

In order to increase the average proton beam power on the anti-proton target and to eliminate the perturbation of the experiments by the MR overpasses, a new injector synchrotron, the Main Injector (MI), was constructed between 1993 and 1999 and MR was decommissioned. MI is a synchrotron of $3.32 \mathrm{~km}$ circumference and a minimum repetition time of $1.4 \mathrm{~s}$. It is located in a new tunnel and provides the protons at $120 \mathrm{GeV}$ for anti-proton production. It also accelerates the antiprotons and the protons from $8 \mathrm{GeV}$ to $150 \mathrm{GeV}$ for injection into the collider. 
For the antiproton production, the full complement of accelerators and storage rings in the injector chain is used. The first accelerator in the injector chain is a $400 \mathrm{MeV} \mathrm{H}^{-}$linear accelerator (linac) operating at $200 \mathrm{MHz}$. Its initial energy was $200 \mathrm{MeV}$ but half of the original drift-tube linac was replaced in 1992-1993 by sidecoupled accelerating structures providing $300 \mathrm{MeV}$. The protons are transferred to the booster synchrotron operating with $15 \mathrm{~Hz}$ repetition rate and are accelerated to a kinetic energy of $8 \mathrm{GeV}$. The intensity is up to $5 \times 10^{12}$ protons per booster pulse. Next the particles are accelerated to $120 \mathrm{GeV}$ in the Main Injector (MI), and hit a solid metal target followed by a lithium lens.

From the emerging secondary particles anti-protons of $8 \mathrm{GeV}$ kinetic energy are selected and their phase space density is stepwise increased in two rings in series, the Debuncher Ring and the Anti-Accumulator Ring; the latter also accumulates the particles. The anti-protons pass then to the latest addition, the Recycler Ring (RR), were they are cooled further and a second step of accumulation takes place. The RR could be fitted into the MI tunnel as its bending and focusing magnets have a small cross-section the magnetic fields being generated by permanent magnets (1.45 $\mathrm{T}$ field strength). This new storage ring has therefore the same circumference as MI; it is in operation since 2004. Due to this elaborate anti-proton source the antiproton production rate has reached nearly $3 \times 10^{11}$ anti-protons $/ \mathrm{h}$. Still the production rate of the antiprotons is rather low compared to proton production. Hence, the injector chain produces and accumulates anti-protons all the time except during the period of filling the Tevatron with protons and antiprotons which takes about $1 \mathrm{~h}$.

When the Tevatron needs a new fill, which happens about every $10-20 \mathrm{~h}$, the linac, the booster and MI produce the 36 proton bunches for the Tevatron, then anti-protons collected in RR are transferred to MI for acceleration to $150 \mathrm{GeV}$ and injection into the Tevatron. The 36 bunches are arranged in 3 trains of 12 bunches. Protons and antiprotons circulate on helical orbits produced by high-voltage electrostatic separators to prevent collisions during injection and acceleration. At top energy, after increasing the focusing in the two collision points equipped with detectors, the separator configuration is modified to bring the beams in collision in these two points [67].

A peak luminosity of $4 \times 10^{32} \mathrm{~cm}^{-2} \mathrm{~s}^{-1}$ has been reached by continuously upgrading all the systems, introducing advanced accelerator technology and stream-lining the operational procedures. The Tevatron has delivered an integrated luminosity of more than $12 \mathrm{fb}^{-1}$ to each of the experiments (CDF and D0) before it ceased operation at the end of September 2011. It was one of the most complex research instruments ever built and will be known for its advances in accelerator physics and technological breakthroughs [68].

\subsubsection{Acknowledgement}

Vladimir Shiltsev (FNAL) has contributed with useful suggestions and pertinent comments to this chapter. Sincere thanks are due to him. 


\subsection{RHIC $^{1}$}

T. Roser

\subsubsection{The RHIC Facility}

With its two independent superconducting rings RHIC is a highly flexible collider of hadron beams ranging from intense beams of polarized protons to fully stripped gold ions [69, 70]. The layout of the RHIC accelerator complex is shown in Fig. 10.1. The collision of $100 \mathrm{GeV} /$ nucleon gold ions probes the conditions of the early universe by producing extreme conditions where quarks and gluons are forming a new state of matter, the strongly interacting quark-gluon plasma. Several runs of high luminosity gold-gold collisions as well as comparison runs using proton, deuteron and copper beams have demonstrated that indeed a new state of matter with extreme density is formed in the RHIC gold-gold collisions. (See also Sect. 11.5).



Fig. 10.1 Layout of RHIC and the injector accelerators. The gold ions are stepwise ionized as they are accelerated to RHIC injection energy

\footnotetext{
${ }^{1}$ This manuscript has been authored by Brookhaven Science Associates, LLC under Contract No. DE-SC0012704 with the U.S. Department of Energy. The United States Government retains and the publisher, by accepting the article for publication, acknowledges that the United States Government retains a non-exclusive, paid-up, irrevocable, world-wide license to publish or reproduce the published form of this manuscript, or allow others to do so, for United States Government purposes.
} 
The RHIC polarized proton collider has opened up the completely unique physics opportunities of studying spin effects in hadronic reactions at high-luminosity high-energy proton-proton collisions. It allows the study of the spin structure of the proton, in particular the degree of polarization of the gluons and anti-quarks, and also verification of the many well-documented expectations of spin effects in perturbative QCD and parity violation in $\mathrm{W}$ and $\mathrm{Z}$ production. The RHIC center-ofmass energy range of $200-500 \mathrm{GeV}$ is ideal in the sense that it is high enough for perturbative QCD to be applicable and low enough so that the typical momentum fraction of the valence quarks is about 0.1 or larger. This guarantees significant levels of parton polarization.

During its 10 years of operation RHIC has greatly exceeded the design parameters for gold-gold collisions, has successfully operated in an asymmetric mode of colliding deuteron on gold with both beams at the same energy per nucleon, and thereby at different rigidities, and successfully completed a comparison run of colliding copper beams with record luminosities. Operation at unequal rigidities of the two colliding beams is a unique feature of RHIC with its two independent rings. The interaction regions are designed to separate the two beams first before they go through their separate final focus triplets. This is shown in Fig. 10.2 for equal species and for the most unequal species of protons and gold beams with a rigidity ratio of 2.47 for equal energy per nucleon. The necessary increase in distance between the interaction point and the final focus triplet limits the achievable luminosity in RHIC.

In addition to heavy ions, RHIC successfully demonstrated its capabilities as a high luminosity polarized proton collider both at 100 and $250 \mathrm{GeV}$ proton beam energy. For most of the heavy ion runs RHIC was operating with beam energies of $100 \mathrm{GeV} /$ nucleon - the gold beam design energy. Additional running at lower beam energy was also accomplished again demonstrating the high level of flexibility of RHIC. Gold collisions at energies much below the RHIC injection energy of $10 \mathrm{GeV} /$ nucleon is allowing the study of the critical point in the quark-gluon phase diagram. Figure 10.3 shows the achieved integrated nucleon-pair luminosities for the many modes of operation of RHIC since its start of operation in 2000. Using nucleon-pair luminosity, which is calculated as the ion-ion luminosity times the

Fig. 10.2 Interaction region geometry in RHIC for equal species (solid line) and the most extreme example of dissimilar species-protons and gold (dashed line)







Fig. 10.3 Integrated nucleon-pair luminosity for the heavy ion (left) and the polarized proton (right) running modes since the start of RHIC operation

Table 10.9 Major parameters of RHIC

\begin{tabular}{l|l}
\hline Parameter & Value \\
\hline Circumference & $3833.845 \mathrm{~m}$ \\
\hline Number of interaction points & 6 \\
\hline Harmonic number, acceleration & 360 \\
\hline Harmonic number, storage & 2520 \\
\hline Typical betatron tunes for $\mathrm{Au}$ & $28.23 / 29.22$ \\
\hline Transition energy $\gamma_{\mathrm{T}}$ & $26.7(\mathrm{Au}), 22.8 \mathrm{(p})$ \\
\hline Maximum magnetic rigidity & $839.5 \mathrm{Tm}$ \\
\hline Total number of dipoles, both rings & 396 \\
\hline Total number of quadrupoles, both rings & 492 \\
\hline Dipole field at $100 \mathrm{GeV} / \mathrm{nucleon}, \mathrm{Au}$ & $3.458 \mathrm{~T}$ \\
\hline Arc dipole effective length & $9.45 \mathrm{~m}$ \\
\hline Arc quadrupole gradient & $71.2 \mathrm{~T} / \mathrm{m}$ \\
\hline
\end{tabular}

number of nucleons in each of the two beam ions, allows the comparison of the different modes of operation properly reflecting the relative statistical relevance of the data samples and also the degree of difficulty in achieving high luminosity.

For RHIC's major parameters and achieved performance parameters see Tables 10.9 and 10.10 , respectively.

\subsubsection{Collider Operation}

\section{Gold-Gold Operation}

Starting with $\mathrm{Au}^{1-}$ from a sputter source the gold ions are stepwise ionized as they are accelerated in the Tandem Van de Graaff, the AGS Booster and the AGS to RHIC injection energy. The electrostatic acceleration in the Tandem Van de Graff 


provides an extremely bright gold beam that can be captured and bunch-merged to provide the necessary bright bunches of $1 \times 10^{9} \mathrm{Au}$ ions with a normalized transverse rms emittance of less than $2.5 \mu \mathrm{m}$ and a total longitudinal emittance of less than $0.3 \mathrm{eVs} /$ nucleon. The final stripping to bare $\mathrm{Au}^{79+}$ occurs on the way to RHIC. Recently the Tandem van de Graff pre-injector has been replaced by an Electron Beam Ion Source (EBIS) followed by an RFQ and IH-Linac. The EBIS can produce very bright high-charge state heavy ion beams by accumulating and stripping heavy ions with a $10 \mathrm{~A}$ space-charge neutralizing electron beam. The new source can produce beams of many species including uranium.

The two RHIC rings, labeled blue and yellow, are intersecting at six interaction regions (IRs). All IRs can operate at a betastar between 2 and $10 \mathrm{~m}$. In two interaction regions, occupied by the two main detectors STAR and PHENIX, the quality of the triplet quadrupoles allows further reduction of betastar to less than $1 \mathrm{~m}$. Typically betastar is about $10 \mathrm{~m}$ at injection energy for all IRs and is then squeezed during the acceleration ramp first to $5 \mathrm{~m}$ at the transition energy $\left(\gamma_{\mathrm{T}}=26.7\right)$, which minimizes the momentum dependence of the transition energy crossing, and then to less than $1 \mathrm{~m}$ for PHENIX and STAR. A typical acceleration cycle consists of filling the blue ring with 111 bunches in groups of 4 bunches, filling the yellow ring in the same way and then simultaneous acceleration of both beams to storage energy. During acceleration the beam bunches are longitudinally aligned but are separated vertically by $10 \mathrm{~mm}$ in the interaction regions to avoid beam losses from beam-beam interaction.

The collision rate is measured using identical Zero Degree Calorimeters (ZDC) at all detectors. The ZDC counters detect at least one neutron on each side from mutual Coulomb and nuclear dissociation with a total cross section of about 10 barns. Typical stores in RHIC last about 4-5 h. Due to intra-beam scattering, which is particularly important for the fully stripped, highly charged gold beams, the initial normalized rms emittance of about $2.5 \mu \mathrm{m}$ grows to about $5 \mu \mathrm{m}$ at the end of the store and a significant amount of beam is lost from the RF bucket. The resulting luminosity lifetime is about $2.5 \mathrm{~h}$.

The reduced number of gold ions compared to typical proton bunches makes it possible to contemplate stochastic cooling of the $100 \mathrm{GeV} /$ nucleon bunched beam. A $6-9 \mathrm{GHz}$ longitudinal and a $5-8 \mathrm{GHz}$ vertical stochastic cooling system was installed using novel high power multi-cavity kickers and the high energy bunched beam was successfully cooled [71]. Figure 10.4 shows the successful cooling of the transverse emittance in the yellow ring compared to the emittance growth in the blue ring without cooling. Both the vertical and horizontal emittance are reduced due to $x-y$ coupling. Figure 10.5 shows the vertex distribution at the end of the store with and without stochastic cooling. It clearly shows a significant increase in luminosity in the central region. The modulation of the vertex distribution is a result of the $200 \mathrm{MHz}$ storage RF system that provides about $4 \mathrm{MV} /$ turn to compress the bunch length and consequently reduce the length of the vertex distribution to better match the acceptance of the collider detectors. Again due to intra-beam scattering the initially narrow distribution widens during the store. A new $2 \mathrm{MV}$, $56 \mathrm{MHz}$ superconducting cavity will provide improved RF focusing that, together 
Fig. 10.4 Transverse emittance evolution in the blue (filled) and yellow (open) RHIC rings with vertical stochastic cooling in the yellow ring [71]

Fig. 10.5 Vertex distribution at the end of a $100 \mathrm{GeV} / \mathrm{n}$ $\mathrm{Au}-\mathrm{Au}$ store with and without longitudinal stochastic cooling


with the longitudinal stochastic cooling, will maintain the short vertex distribution throughout the store.

With the two planes of stochastic cooling operational in both rings a peak luminosity at PHENIX and STAR of up to $40 \times 10^{26} \mathrm{~cm}^{-2} \mathrm{~s}^{-1}\left(160 \times 10^{30} \mathrm{~cm}^{-2} \mathrm{~s}^{-1}\right.$ nucleon-pair luminosity) with an average store luminosity of $20 \times 10^{26} \mathrm{~cm}^{-2} \mathrm{~s}^{-1}$ : ten times the original RHIC design average store luminosity. The full stochastic cooling system will include also the horizontal plane and should result in another doubling of the luminosity.

The total gold beam intensity in RHIC was initially limited by vacuum breakdowns in the room temperature sections of the RHIC rings [72]. This pressure rise is associated with the formation of electron clouds, which in turn appear when the bunch peak intensity is high around transition and after bunch compression, and when the bunch spacing is below about $200 \mathrm{~ns}$. This situation was greatly improved by installing vacuum pipes with an internal coating of non-evaporative getter (NEG) that is properly activated. The resulting residual static pressure is about $10^{-11}$ Torr. The NEG coating acts as a very effective distributed pump and also suppresses electron cloud formation due to its low secondary electron yield.

Very fast single bunch transverse instabilities that develop near transition, where the chromaticity needs to cross zero, originally limited bunch intensity and is still responsible for occasional emittance growth, especially towards the end of bunch trains. The instability can be stabilized using octupoles [73] and the instability 
threshold can be increased by lowering the peak current during transition crossing. This instability has a growth rate faster than the synchrotron period and is similar to a beam break-up instability. The instability is also enhanced by the presence of electron clouds [74].

\section{Deuteron-Gold Operation}

Colliding $100 \mathrm{GeV} /$ nucleon deuteron beam with $100 \mathrm{GeV} /$ nucleon gold beam will not produce the required temperature to create a new state of matter and therefore serves as an important comparison measurement to the gold-gold collisions. The rigidity of the two beams is different by about $20 \%$, which results in different deflection angles in the beam-combining dipoles on either side of the interaction region. This requires a non-zero angle at the collision point, which slightly reduces the available aperture.

The injection energy into RHIC was also the same for both beams requiring the injector to produce beams with different rigidity. With same energy beams throughout the acceleration cycle in RHIC the beams can remain cogged. Without this the effect of the time-modulated long-range beam-beam interaction in the IRs would lead to rapid beam loss. The typical bunch intensity of the deuteron beam was about $1 \times 10^{11}$ with a transverse rms emittances of about $2 \mu \mathrm{m}$ and a total longitudinal emittance of $0.3 \mathrm{eVs} /$ nucleon. The gold beam parameters were similar to the gold-gold operation described above. Recently the ring with the gold beam was operated with increased transverse focusing to reduce the effect of intra-beam scattering on the transverse emittance. As a beneficial side effect the two beams crossed transition at different times, which reduced the development of the fast transverse instability. A peak luminosity of $27 \times 10^{28} \mathrm{~cm}^{-2} \mathrm{~s}^{-1}$ $\left(100 \times 10^{30} \mathrm{~cm}^{-2} \mathrm{~s}^{-1}\right.$ nucleon-pair luminosity) and store-averaged luminosity of $14 \times 10^{28} \mathrm{~cm}^{-2} \mathrm{~s}^{-1}$ was reached at the IRs with a $0.85 \mathrm{~m}$ betastar.

\section{Polarized Proton Operation}

Figure 10.6 shows the layout of the RHIC accelerator complex highlighting the components required for polarized beam acceleration. The 'Optically Pumped Polarized Ion Source' [75] is producing about $10^{12}$ polarized protons per pulse. A single source pulse is captured into a single bunch, which is sufficient beam intensity to reach in RHIC the nominal bunch intensity of $2 \times 10^{11}$ polarized protons.

In the AGS two partial Siberian snakes are installed, an iron-based helical dipole that rotates the spin around the longitudinal direction by $11^{\circ}$ and a superconducting helical dipole that can reach a $3 \mathrm{~T}$ field and a spin rotation of up to $45^{\circ}$. A view down the magnet gap is shown in Fig. 10.7. With the two partial snakes placed with one third of the AGS ring between them all vertical spin resonances are avoided up to the required RHIC transfer energy of $23.8 \mathrm{GeV}$ as long as the vertical betatron tune is placed at 8.98 , very close to an integer [76]. With an $80 \%$ polarization from the source $65 \%$ polarization was reached at AGS extraction. The remaining polarization loss in the AGS comes from weak spin resonances driven by the horizontal motion of the beam [77]. It is planned to overcome them by quickly shifting the betatron tune during resonance crossing. 


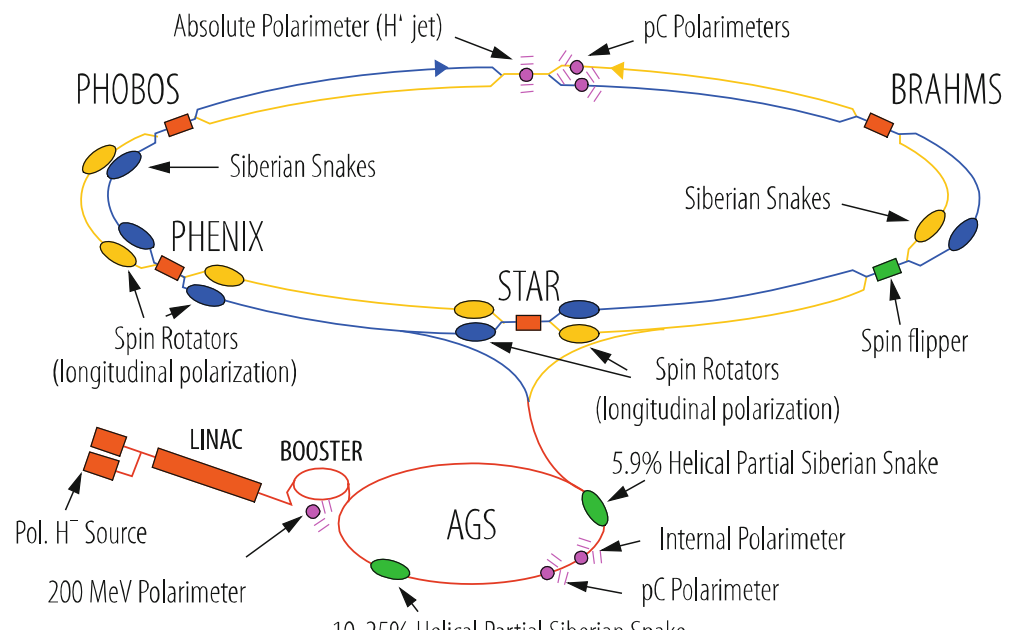

10-25\% Helical Partial Siberian Snake

Fig. 10.6 The RHIC accelerator complex with the elements required for the acceleration and collision of polarized protons highlighted



Fig. 10.7 View down the magnet gap of the warm, iron-based helical partial Siberian snake of the AGS

The full Siberian snakes [78], two for each ring, and the spin rotators, four for each collider experiment, in RHIC each consist of four $2.4 \mathrm{~m} \mathrm{long}, 4 \mathrm{~T}$ superconducting helical dipole magnet modules each having a full $360^{\circ}$ helical twist. Figure 10.8 shows the orbit and spin trajectory through a RHIC snake. The two Siberian snakes are installed in the location of the missing dipole of the dispersion suppression section and the orbit angle is exactly $180^{\circ}$ in between the two snakes.

The spin rotation axis of the two snakes is pointing $45^{\circ}$ in and out, respectively, relative to the direction of the beam resulting in the required $90^{\circ}$ angle between the 




Fig. 10.8 Orbit and spin tracking through the four helical magnets of a Siberian Snake. The spin tracking shows the reversal of the vertical polarization. The spin rotation axis is in the horizontal plane and is pointing $45^{\circ}$ away from the beam direction

spin rotation axes. This configuration yields a spin tune $Q_{\mathrm{s}}=0.5$ and a vertical stable spin direction around the ring. Here, spin tune is defined as the number of spin precessions in one orbital revolution. Since the betatron tunes in circular accelerators are kept away from half integer to keep the beam stable, both intrinsic and imperfection depolarizing spin resonances at $G \gamma=k P \pm Q_{\mathrm{y}}$ and $G \gamma=k$ are avoided [79]. Here, $G$ is the anomalous $g$ factor, $\gamma$ is the Lorentz factor, $k$ is an integer, $P$ is the super-periodicity of the accelerator, and $Q_{\mathrm{y}}$ is the vertical betatron tune.

The accurate measurement of the beam polarization is required for set-up and operation of the polarized proton collider. Very small angle elastic scattering in the Coulomb-Nuclear interference region offers the possibility for an analyzing reaction with a high figure-of-merit, which is not expected to be strongly energy dependent [80]. For polarized beam commissioning in RHIC an ultra-thin carbon ribbon is used as an internal target, and the recoil carbon nuclei are detected to measure both vertical and radial polarization components. The detection of the recoil carbon with silicon detectors using both energy and time-of-flight information shows excellent particle identification. It was demonstrated that this polarimeter can be used to monitor polarization of the high energy proton beams in an almost non-destructive manner and that the carbon fiber target could be scanned through the circulating beam to measure beam and polarization profiles. A polarized atomic hydrogen jet was also installed as an internal target for small angle proton-proton scattering which allows the absolute calibration of the beam polarization to better than $5 \%$.

Figure 10.9 shows circulating beam current, luminosity and measured circulating beam polarization of a typical store with a beam energy of $100 \mathrm{GeV}$. The maximum peak luminosity achieved with $100 \mathrm{GeV}$ proton beams is about $50 \times 10^{30} \mathrm{~cm}^{-2} \mathrm{~s}^{-1}$ and store beam polarization of about $55 \%$ calibrated at $100 \mathrm{GeV}$ with the absolute polarimeter mentioned above. To preserve beam polarization in RHIC during acceleration and storage the vertical betatron tune has to be controlled to better than 0.005 and the orbit has to be corrected to better than $1 \mathrm{~mm}$ rms to avoid depolarizing "snake" resonances.

During acceleration from $100 \mathrm{GeV}$ to the maximum RHIC energy of $250 \mathrm{GeV}$, several very strong depolarizing spin resonances need to be crossed [81]. In a first running period an average store polarization of $34 \%$ was measured at $250 \mathrm{GeV}$ again calibrated with the absolute polarimeter. The peak luminosity 



Fig. 10.9 Circulating beam in the blue and yellow ring, luminosity at STAR (red), as well as the measured circulating beam polarization in the blue and yellow RHIC ring (blue (dark) and yellow (light) lines and symbols, respectively) for a typical store with $100 \mathrm{GeV}$ beam energy

reached was about $85 \times 10^{30} \mathrm{~cm}^{-2} \mathrm{~s}^{-1}$ and the average store luminosity was about $55 \times 10^{30} \mathrm{~cm}^{-2} \mathrm{~s}^{-1}$. The goal for operation of RHIC with polarized $250 \mathrm{GeV}$ proton beams is a peak luminosity of $200 \times 10^{30} \mathrm{~cm}^{-2} \mathrm{~s}^{-1}$ with a beam polarization of $70 \%$.

Head-on beam-beam effects are the main limitation for increasing the luminosity in proton-proton operation. The maximum beam-beam parameter reached was 0.0065 per IP with $100 \mathrm{GeV}$ beams when operating with beams in collision at two detector IPs. This value is somewhat smaller than what was reached at the Tevatron proton-antiproton collider probably due to the smaller momentum aperture available at RHIC as a result of the dipole first IR design. Additional sextupole circuits are available to correct non-linear chromaticity and the beta-function momentum dependence. In the future it is planned to mitigate the beam-beam effects with the installation of two electron lenses in RHIC. Space is available to partially compensate the beam-beam effect with an opposite charge electron beam separated from the proton-proton collisions by the correct phase advance. With the electron lenses and also a more intense polarized proton source it is expected that the protonproton luminosity could be increased by about a factor of two.

\section{Recent Progress}

The performance of RHIC has been continuously improved with store-averaged luminosity reaching $87 \times 10^{26} \mathrm{~cm}^{-2} \mathrm{~s}^{-1}$, exceeding the design luminosity by more 
than a factor of 40, and polarized proton operation at $510 \mathrm{GeV}$ center-of-mass energy with peak luminosity of $250 \times 10^{30} \mathrm{~cm}^{-2} \mathrm{~s}^{-1}$ with store-averaged beam polarization of up to $60 \%$. Major upgrades to RHIC included electron lenses to compensate for head-on beam-beam interactions [82] and a superconducting storage RF system. In preparation for operation at very low center-of-mass energies with beam energies below the RHIC injection energy bunched beam electron cooling of both RHIC ion beams is being installed [83].

\subsection{Electron Accelerators and Electron-Positron Colliders}

\section{J. Seeman}

\subsubsection{Cyclotrons}

Cyclotrons are one of the first accelerators invented and use a fixed magnetic field and a radiofrequency (RF) cavity to accelerate electrons in ever increasing orbits [84]. The electron beam is injected into the center of a circular magnetic field region between magnet poles and then made to circulate. These electrons are accelerated twice on each turn and are extracted at high energy near the outer edge of the accelerator. The cyclotron can produce continuous beams leading to high power applications. The first cyclotron was built at Berkeley in 1931 with a diameter of $11 \mathrm{~cm}$. Many more cyclotrons were to follow over the years. The TRIUMF cyclotron in use now is located in Vancouver, Canada, and has a diameter of $18 \mathrm{~m}$ and has a 4000 ton magnet. The beam energy is limited by the field strength and the diameter of the magnetic pole. Cyclotrons are mainly used to produce high beam power for science, for materials analysis, and industrial processing applications. Two cyclotrons are shown in Fig. 10.10.

\subsubsection{Synchrotrons}

A synchrotron is a fixed circumference accelerator that increases the electron beam energy using RF accelerating cavities but keeps the radius constant by increasing the magnetic fields. The field used to bend the beam in a circle is ramped in proportion to the beam energy [85]. The beam is injected at low energy and magnetic fields, accelerated, and then extracted at high energy and magnetic fields. The highest beam energy is limited by the strength of the magnetic field and the diameter of the accelerator which can be over $1 \mathrm{~km}$ and is usually limited by the site or its building. Synchrotrons are pulsed machines due to the cyclic ramping of the magnetic fields. The RF system accelerates the beam during the ramp and keeps the beam bunched. 




Fig. 10.10 On the left is the first electron cyclotron constructed at the Berkeley in 1931 (courtesy LBNL Berkeley, CA). The right photograph is a modern cyclotron at PSI (courtesy PSI Lausanne, Switzerland)


Fig. 10.11 The left photograph is the $12 \mathrm{GeV}$ electron synchrotron at Cornell (courtesy Wilson Laboratory Ithaca, NY) used as an injector into the CESR storage ring collider, also shown. The right photograph is the Advanced Light Source injector synchrotron (courtesy LBNL Berkeley, CA)

The magnets are often divided into separated units to allow simplified construction including dipoles for bending, quadrupoles for beam focusing, sextupoles for chromatic corrections, and correction dipoles for trajectory tuning. The quadrupoles often form an alternating focus and defocusing magnetic lattice around the ring to produce "strong focusing" which reduces the beam's excursions. As a result, the cross sections of the magnet gaps can be made significantly smaller and less expensive. The radiofrequency RF system can have a large range of frequencies and cavity designs. A vacuum system in the milli-Torr to nano-Torr level is also needed. Synchrotrons are often used as injectors for higher energy accelerators and storage rings. In Fig. 10.11 are shown the electron synchrotrons at Cornell $(12 \mathrm{GeV}, \sim 756 \mathrm{~m})$ and the injector at the Berkeley Advanced Light Source. 


\subsubsection{Electron Positron Circular Colliders}

In the late 1950s the accelerator community realized that colliding beams head-on would increase the center of mass energy significantly over fixed target collisions and could significantly expand the reach for new particles and physics. The goal was to design an accelerator that could provide enough centre of mass energy to make new physics, high enough beam currents and small enough collision spot sizes to provide a sufficient data rate, and to provide a volume for the particle physics detector surrounding the collision point with backgrounds sufficiently low to allow clean data collection. A world wide effort was initiated to design these accelerators and has continued to today [86-88]. At least 25 electron-electron or electronpositron colliders have been built and operated for particle physics over the past 50 years with ever increasing beam currents, luminosity, energy, and sophistication. Several colliders are shown in Fig. 10.12. In Table 10.11 are listed in chronological order these 24 colliders along with several of their technical parameters. Following the table are descriptions of each of the colliders discussing several of their



Fig. 10.12 The ADA collider on the top left (courtesy INFN Frascati, Italy). Top right is the PEPII B-Factory collider (courtesy SLAC Menlo Park, CA). The LEP collider is shown on the bottom left (courtesy CERN Geneva, Switzerland). SuperKEKB is shown on the bottom right (courtesy KEK Tsukuba, Japan) 


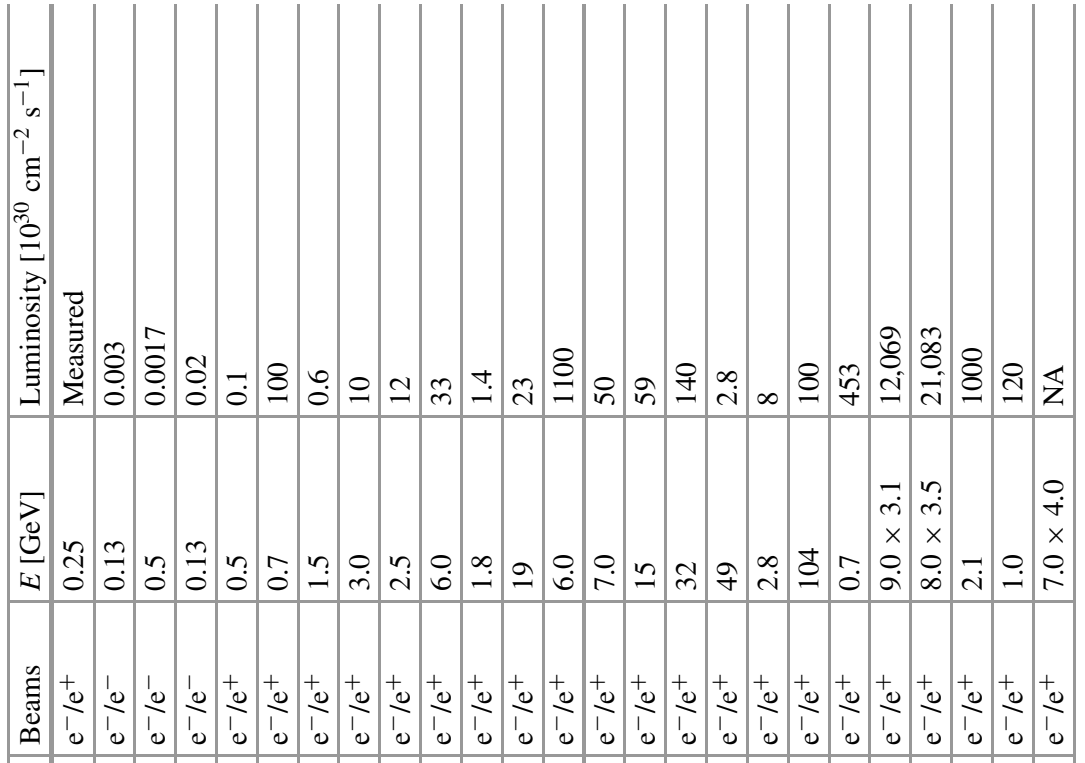




कृष्ठ




ڤี

(5)

एँ 
unique features and results. The intent is to illustrate the advancement of collider technology over the years. Several of the technical advances are high power RF systems, superconducting RF systems, high field magnets, superconducting magnets, high field permanent magnets, ultra-high vacuum systems, low emittance beam lattices, complex interaction region designs, and sophisticated diagnostics and controls. Many of the colliders in later life became synchrotron radiation sources and several serve in this capacity today.

\subsubsection{ADA}

ADA at Frascati was a pioneering electron-positron collider [89]. This collider was used to study stored beam parameters, injection, beam lifetime, and collisions. Injection of electrons and positrons was made by converting $1 \mathrm{GeV}$ gamma rays in a small target inside the ring. The mean lifetime was consistent with gas bremsstrahlung. With the electrons stored, the whole magnet was rotated about a horizontal axis to inject positrons without losing electrons. A pulsed kicker was used to reduce the betatron oscillations of the injected particles. Luminosity was measured. The effects of Touschek scattering was discovered and studied.

\subsubsection{VEP-1}

VEP-1 at BINP was an early electron-electron collider used to study elastic scattering and double bremsstrahlung experiments [90]. This accelerator demonstrated the possibility to carry out colliding beam experiments for particle physics.

\subsubsection{CBX}

The Princeton-Stanford Colliding Beam Experiment CBX on the Stanford campus was an electron-electron collider used to study elastic scattering and double bremsstrahlung experiments [91]. G. O'Neill on this team proposed that radiation damping for a stored electron beam could help with injection and emittance reduction. The beam-beam tune shift limit was seen in this machine on the level of 0.02-0.05. CBX saw indications of the single beam resistive wall instability. Electron currents up to $1 \mathrm{~A}$ were stored. This accelerator also demonstrated the possibility to carry out colliding beam experiments for particle physics.

\subsubsection{VEPP-2}

VEPP-2 at BINP was one of the first electron-positron collider in the world [92, 93]. The rho and phi meson parameters were measured using annihilation into two pions followed later by omega and phi meson decay parameters in the vector meson region 
and the first observation of two-photon pair production. This collider was quickly rebuilt as VEPP-2M.

\subsubsection{ACO}

ACO at Orsay was an early electron-positron collider used to demonstrate collider principles [94]. This accelerator had six dipoles with quadrupoles in between. The ring was later used as a synchrotron light source demonstrating ring based FEL science. This accelerator gave way to the DCI collider a few years later.

\subsubsection{ADONE}

ADONE at Frascati was a one ring collider with a $50 \mathrm{MHz}$ RF system [95]. ADONE had longitudinal phase feedback to damp beam instability modes, a $4 \mathrm{kG}$ detector solenoidal field (MEA), magnet shunts to adjust the beta functions in the ring, and adjustable damping partition numbers generated by an RF frequency change. Injection was from a linac with an energy ramp after the fill. The measured luminosity increased a little over the fourth power of the energy. ADONE later confirmed the existence of the J/Psi.

\subsubsection{CEA}

The CEA Cambridge Electron Colliding Beam Facility used a linac for injection at $120 \mathrm{MeV}\left(\mathrm{e}^{+}\right)$and $240 \mathrm{MeV}\left(\mathrm{e}^{-}\right)$and then ramped both beams in the ring to full energy [96]. The accelerator technology advancements for the CEA are the first demonstration of a low beta insertion at the IP, single-turn pulsed orbit switching into an interaction point (IP) magnetic bypass, switching from energy cycling to dc operation at full energy and the addition of two damping magnets to redistribute the radial damping so that both betatron and synchrotron oscillations were damped. In particle physics the CEA measured that the $R$ cross section ratio rose at higher center of mass energy, hinting at many discoveries to come later at other colliders.

\subsubsection{SPEAR}

SPEAR at SLAC was designed as a two ring collider but due to funding only one ring was built. Injection was at full energy from the SLAC 2-mile linac with a positron source in linac sector 11 [97]. The ring lattice was extremely flexible in the choice of operating tunes, dispersion, and beta values the IPs. Transverse horizontal and vertical instabilities (head-tail) were observed at about $0.5 \mathrm{~mA}$ per bunch which were cured by a positive chromaticity. The luminosity varied as the fourth power of 
the beam energy. SPEAR was very productive in particle physics with the discovery of the J/Psi and tau. SPEAR has been upgraded to a medium emittance light source.

\subsubsection{VEPP-2M}

VEPP-2M at BINP reached a luminosity 100 times VEPP-2. VEPP-2 served for a while as the injector $[93,98]$. The ring operated with a superconducting wiggler with an $8 \mathrm{~T}$ field that was used to increase the radial emittance, decrease the damping time, increase the beam-beam tune shift for a higher luminosity, and also for suppression of intra-beam scattering. The collider could generate and use polarized beams. The particle physics results are many. Round beams have been studied at VEPP-2M to try to reduce beam-beam effects and, thus, increase the allowed beambeam tune shift. Round beams required the use of solenoidal focusing at the IPs.

\subsubsection{DORIS}

DORIS at DESY started as a two ring collider with beams brought into collision with vertical dipoles in the IR and had a vertical crossing angle [99]. Injection was from a linac and a booster synchrotron. The single bunch currents in the collider were limited by higher parasitic modes in the RF cavities. The luminosity was limited by effects of the vertical crossing angle. DORIS was subsequently converted to a single ring collider with head-on collisions and went on to do many years of B meson physics.

\subsubsection{DCI}

DCI at Orsay was a two ring collider designed for high energy physics studies of charged and neutral particles and also of two photon physics [100]. The two rings were mounted one above the other and the beams were brought into collision with vertical bends near the IR. Both rings could store both $\mathrm{e}^{-}$and $\mathrm{e}^{+}$. The rings could operate with either two bunch collisions or four bunch collisions (opposite in both rings). The four bunch collisions aimed at charge cancelation of the beambeam effects allowing higher beam-beam tune shifts and thus higher luminosity. The four beam scheme in reality partially worked but was strongly limited by incoherent and coherent beam-beam modes. This topic of four beam collisions has been theoretically studied for many years since. The DCI collider mostly operated in a one bunch mode in each ring (out of time) generating twice the luminosity. The peak luminosity scaled as the energy squared. 


\subsubsection{PETRA}

PETRA at DESY had four interaction points and operated up to an energy of $24 \mathrm{GeV}$ per beam with $2 \times 2$ bunches [101]. Additional seven cell RF cavities were installed over the years to achieve these energies. Second harmonic cavities $(1 \mathrm{GHz})$ were installed to reduce the bunch length, cure a vertical single bunch instability and reduce several synchrotron-betatron resonances. The free space for the detector was reduced to $\pm 4.45 \mathrm{~m}$ using mini-beta insertions allowing a vertical beta of $6 \mathrm{~cm}$. The injected positrons were predamped in the accumulator storage ring PIA. PETRA is known for the discovery of the Gluon and for QCD studies. PETRA has been upgraded to a low emittance light source.

\subsubsection{CESR}

CESR at Cornell operated for B-meson studies for 20 years [102, 103]. The particle physics results included $V_{\mathrm{ub}}$ observations of "penguin" modes, $\mathrm{b} \rightarrow \mathrm{s} \gamma$ decays, CKM matrix constraining the unitarity triangle, and B mass and lifetime measurements. Injection was from a $200 \mathrm{MeV}$ linac and a $12 \mathrm{GeV}$ synchrotron. With electrostatic plates installed, CESR could collide up to 27 bunches separated in the accelerator arcs by what is now called a "Pretzel orbit" that was used to suppress parasitic beam-beam collisions and the related tune shifts. Several other colliders went on to use this technique to increase the number of bunches. It was discovered at CESR that a horizontal tune just above the half integer $(<0.51)$ increased significantly the beam-beam limit allowing higher luminosity. Superconducting single-cell cavities with HOM damping were installed in CESR allowing up to $325 \mathrm{~mA}$ beams each of electrons and positron to the stored. The CESR interaction region used a combination of permanent-magnet and superconducting technologies for the vertically focusing quadrupoles. A $\pm 2.5 \mathrm{mrad}$ uncompensated IP crossing angle was ultimately used. Superconducting wigglers were later installed to allow operation at lower energy at the charm threshold. CESR is presently being used as a light source and as a test accelerator to study low emittance damping rings and electron cloud physics.

\subsubsection{VEPP-4}

VEPP-4 at BINP has been colliding beams for a long time including two modernizations [104]. Over the years, the vertical beta at the IP was reduced to $5 \mathrm{~cm}$, superconducting wigglers were added to increase the luminosity, and a superconducting RF cavity was installed for bunch length reduction allowing the use of the lower IP beta. Bunch currents were limited by beam induced wakefields in the vacuum chambers at the level of $17 \mathrm{~mA}$. Eight pairs of electrostatic separation plates allow two bunch operation in a Pretzel scheme. A transversely polarized beam could be injected into VEPP-4 from VEPP-3 for accurate measurements of the masses of 
the upsilon family particles. Other particle physics studied at VEPP-4M included precise tau and J/Psi mass measurements and two photon physics.

\subsubsection{PEP}

PEP at SLAC operated with three bunches per beam up to $14 \mathrm{GeV}$ delivered to six interaction points [105]. PEP had aluminum vacuum chambers and aluminum RF cavities coated with TiN to suppress breakdowns. The head-tail microwave beam instability was studied extensively at PEP and cures investigated. Collisions with 1 , 2, 4, and 6 IPs allowed studies of the beam-beam limits with different damping times per collision. The particle physics results at PEP included the first measurements of the tau lepton lifetime, the discovery that the B meson lifetime was unexpectedly long, analysis of jet structures, and the measurements of lifetimes and properties of charm and bottom hadrons.

\subsubsection{Tristan}

Tristan at KEK collided $2 \times 2$ bunches in four interaction points and was designed to search for high mass resonances [106]. The injector was a $2.5 \mathrm{GeV}$ linac and a $377 \mathrm{~m}$ accumulator ring ramped to the $8 \mathrm{GeV}$ injection energy. Tristan was the first large accelerator to use extensive superconducting RF technology.

\subsubsection{SLC}

The SLAC Linear Collider SLC was the first (and so far only) linear collider constructed. It was built to precisely measure the properties of the $Z^{0}$ meson. It was the first to measure the width of the $Z^{0}$ indicating only three families of light quarks and neutrinos. It also provided a precise indirect constraint on the Higgs mass [107]. The SLC collided single $\mathrm{e}^{+}$and $\mathrm{e}^{-}$bunches at $120 \mathrm{~Hz}$ with $80 \%$ longitudinal $\mathrm{e}^{-}$polarization at the IP coming from a polarized strained GaAs photo-gun. Other accelerator advances include BNS emittance damping in the linac, reduced emittances from $\mathrm{e}^{-}$and $\mathrm{e}^{+}$damping rings, pulse-by-pulse IP position feedback, and a positron source and target with one-to-one $\mathrm{e}^{-}$in to $\mathrm{e}^{+}$out conversion rate. About $10^{13}$ positrons were made and collided per second.

\subsubsection{BEPC}

BEPC at IHEP was built to produce tau and charm particle physics [108]. BEPC was a single ring collider with two collision points reaching $2.5 \mathrm{GeV}$ per beam. The single bunch current was up to $22 \mathrm{~mA}$ and $140 \mathrm{~mA}$ in multi-bunch mode. Injection was from a full energy linac with two transport lines. A mini-beta optics 
at the IP using permanent magnet quadrupoles $(0.5 \mathrm{~m}$ long) was installed reaching a vertical beta of $8.5 \mathrm{~cm}$. Higher RF voltage was used to reduce the bunch length to match. BEPC measured precisely the tau lepton mass to $0.2 \mathrm{MeV}$ out of a mass of $1777 \mathrm{MeV}$.

\subsubsection{LEP}

LEP at CERN has four collision points and is the largest $(27 \mathrm{~km})$ and highest energy (104.5 GeV per beam) collider built to date [109]. The particle physics completed at LEP included precise measurements of $\mathrm{Z}$ and $\mathrm{W}$ bosons, determination of the number of light neutrinos to be three, and exclusion of the Higgs mass below $114 \mathrm{GeV}$. To reduce power usage five cell RF cavities with attached spherical copper storage cavities were used to reach about $80 \mathrm{GeV}$. To reach $104.5 \mathrm{GeV}$ additional superconducting RF cavities were added incorporating sputtered $\mathrm{Nb}$ on $\mathrm{Cu}$ surfaces. The bending dipoles in LEP needed only a low field so the steel laminations were spaced by concrete filler material. At the $\mathrm{Z}$ resonance the luminosity was limited by the beam-beam effect. At higher energies the luminosity limit was the available RF power. A Pretzel orbit scheme ( 8 and later 12 bunches) was used to increase the luminosity. At high energy, a low emittance optics was implemented. A beam-beam tune shift of about 0.083 as reached at $98 \mathrm{GeV}$.

\subsubsection{DAFNE}

DAFNE at Frascati was built to make precision measurements of $\mathrm{K}$ meson physics. Injection is made with a full energy linac and damping ring [110]. DAFNE operates with damping wigglers to decrease the emittances and shorten the damping time. A crab waist scheme was installed in the IP for increased luminosity and for tests of the SuperB IP concept [111]. Rolled permanent-magnet IP quadrupoles were used to compensate the detector solenoidal field.

\subsubsection{PEP-II}

PEP-II at SLAC was an asymmetric collider with two rings made to measure the properties of the $\mathrm{b}$ quark sector, the $\mathrm{CP}$ violation in the $\mathrm{B} \overline{\mathrm{B}}$ system, and to confirm the CKM matrix [112]. Injection was made at full energy at up to $30 \mathrm{~Hz}$ from the SLAC $3 \mathrm{~km}$ linac with the SLC $\mathrm{e}^{-}$and $\mathrm{e}^{+}$damping rings and either $\mathrm{e}^{-}$or $\mathrm{e}^{+}$accelerated on any given linac pulse. Accelerator advances at PEP-II include head-on asymmetric collisions at one IR, large bore permanent-magnet IP dipole and quadrupoles, local beta beats to correct chromaticity in the IP, fast IP position feedback, and bunch-by-bunch transverse and longitudinal feedbacks. The nearest final focus quadrupoles were inside the detector leaving a free space to the IP of about $0.5 \mathrm{~m}$ on each side. PEP-II holds the world's record of stored positrons at 
3.2 A and for electrons at 2.1 A. PEP-II was the first collider to allow top-up injection (only a few $\mathrm{Hz}$ were needed) to keep the beam currents and luminosity constant, all with full continuous data collection by the particle physics detector.

\subsubsection{KEKB}

$\mathrm{KEKB}$ at KEK was an asymmetric two ring collider made to measure $\mathrm{CP}$ violation in the $B \bar{B}$ system and to confirm the CKM matrix [113]. Injection was made at full energy with the KEK J-shaped linac with either $\mathrm{e}^{-}$or $\mathrm{e}^{+}$injected at $50 \mathrm{~Hz}$ with a several minute switch time between modes. KEKB had a lattice with a $5 \pi / 2$ phase advance to reduce the emittance well below that of a FODO cell. It had a crossing angle IP, used ARES RF copper cavities with an attached energy storage cell, and superconducting RF cavities to suppress longitudinal modes. Transverse bunch-bybunch feedbacks were used to suppress instabilities. KEKB was the first collider to use superconducting crab cavities to reduce the effects of crossing angle collisions. KEKB also had top-up injection of both beams. KEKB holds the world's record for highest luminosity at $2.1 \times 10^{34} \mathrm{~cm}^{-2} \mathrm{~s}^{-1}$.

\subsubsection{BEPC-II}

BEPC-II at IHEP was built to provide tau and charm particle physics as a factory and also have synchrotron radiation production $[114,115]$. BEPC-II is a double ring collider with one collision point reaching up to $2.1 \mathrm{GeV}$ per beam with 93 bunches. The RF system has two superconducting single cavities at $500 \mathrm{MHz}$. The luminosity was recently limited by longitudinal instability from HOMs but was cured by a bunch-by-bunch longitudinal feedback system. BEPC-II has delivered several billion J/Psi events to the BES-III detector.

\subsubsection{VEPP-2000}

VEPP-2000 at BINP is a recent $\mathrm{e}+\mathrm{e}-$ collider and has a single ring with two detectors and twofold symmetry [116, 117]. The particle physics being done at VEPP-2000 is tau and psi measurements and two gamma-physics. Injection comes from a $900 \mathrm{MeV}$ booster synchrotron with one beam at a time. A round beam concept was applied in the ring design where a particle's angular momentum ( $M=x z^{\prime}-z x^{\prime}=$ constant) is conserved yielding an enhancement of the beam's dynamic stability even with nonlinear effects of the beam-beam force included. This scheme requires equal emittances, equal small fractional tunes, equal betas at the IP, and no betatron coupling in the collider arcs. In practice with collisions for the detectors, only small adjustments in the tunes are needed to arrive at good luminosity conditions at the beginning of a fill. Observations show that a beam-beam 
parameter of 0.13 has been achieved and that round beams give a solid luminosity enhancement.

\subsubsection{SUPERKEKB}

SuperKEKB at KEK is an asymmetric two ring collider upgraded from KEKB and made to measure $\mathrm{CP}$ violation in the $\mathrm{B} \overline{\mathrm{B}}$ system to extend the understanding of the CKM matrix [118]. This collider has a 7.0 GeV High Energy Ring HER for e- and a 4.0 GeV Low Energy Ring LER for $\mathrm{e}+$. The luminosity goal is $8 \times 10^{35} \mathrm{~cm}^{-2} \mathrm{~s}^{-1}$. The design beam currents will be doubled from KEK to $2.6 \mathrm{~A}$ on $3.6 \mathrm{~A}$. The nano-beam scheme for increased luminosity decreases the overlapping length of colliding particles to about $0.25 \mathrm{~mm}$ with a $41.4 \mathrm{mrad}$ half crossing angle [111]. The interaction region beta functions will be about $30 / 0.3 \mathrm{~mm}(\mathrm{~h} / \mathrm{v})$ with a bunch length of about $5.5 \mathrm{~mm}$. The two rings without the interaction region were commissioning in 2016 where $1.01 \mathrm{~A}$ of e- were successfully stored in the HER and $0.87 \mathrm{~A}$ of e+ stored in the LER. The installation of the interaction region will be completed in early 2018 when luminosity commissioning will begin.

\subsection{Asymmetric B-Factories}

K. Oide

\subsubsection{Physics Motivation}

The idea of asymmetric B-factories was first introduced by P. Oddone in 1987 [119] to collide $e^{+} e^{-}$beams with different energies to measure the CP-asymmetry between the decay of $B_{0}$ and $\bar{B}_{0}$ mesons. The asymmetry of the energies of two beams boosts the generated particles longitudinally, then the difference of the decay time can be measured by the difference of the vertices, which was expected to be in about an order of $100 \mu \mathrm{m}$. The center-of-mass energy of the collision is set to the $\Upsilon(4 S)$ resonance at $10.58 \mathrm{GeV}$. A very high luminosity around $10^{34} \mathrm{~cm}^{-2} \mathrm{~s}^{-1}$ was required, which was more than 100 times higher than what had been achieved in colliders by that time.

\subsubsection{Double Ring Collider}

There may be several ways to realize the asymmetric collision. One way is to build a linear-linear or a ring-linear collider. Such a linear machine needs a very strong 
focusing $\beta_{y}^{*} \sim 100 \mu \mathrm{m}$ to achieve the luminosity, then the bunch length must be as short as $\beta_{y}^{*}$ to avoid the hour-glass effect. The bunch length itself can be obtained by bunch compressors, but the associated energy spread degrades the effective luminosity, since the width of the resonance $\Upsilon(4 S)$ is only $20 \mathrm{MeV}\left(2 \times 10^{-4}\right)$. A huge damping ring would be necessary to realize such a short bunch length and a small energy spread simultaneously. Thus linear collision schemes seemed difficult.

As for the double-ring collision, a question is the sizes of the rings. If one can collide a large high energy ring (HER), for instance at $25 \mathrm{GeV}$, with a small low energy ring (LER) at $1.2 \mathrm{GeV}$, the total cost will be saved, assuming an existing tunnel for such a high energy ring. It was pointed out [120] that the collision of rings with different circumferences has somewhat fundamental difficulty: if two rings have the ratio of circumferences $m: n(m>n)$, the periodicity of the system becomes very long, i.e., $\operatorname{LCM}(m, n) / m$ times the revolution period of the larger ring. Then both rings will have dense resonance lines in the tune space which reduces the operable area, especially with a certain amount of the beam-beam tune shift. Thus collision of rings with different circumferences seemed difficult. Therefore only the double ring collider scheme with equal cicumferences remained.

Two projects of the asymmetric B-factories, PEP-II [121] at SLAC and KEKB [122] at KEK, were approved and started the construction by 1994 . Both projects utilized the components and facilities of their previous generation colliders, PEP and TRISTAN, and built the BaBar and Belle detectors, respectively. Both machines started the collision experiments in 1999 and stopped the operation in April 2008 (PEP-II) and June 2010 (KEKB). Table 10.12 lists the main machine parameters corresponding to their best records $[123,124]$. Both colliders achieved higher performance than their designs, and experimentally verified the Kobayashi-Maskawa model to bring the 2008 Nobel Prize in Physics.

\subsubsection{Luminosity}

The luminosity $\mathscr{L}$ of an asymmetric ring collider can be expressed by the following expression:

$$
\mathcal{L}=\frac{\gamma_{ \pm}}{2 e r_{e}}\left(1+\frac{\sigma_{y}^{*}}{\sigma_{x}^{*}}\right)\left(\frac{I \xi_{y}}{\beta_{y}^{*}}\right)_{ \pm}\left(\frac{R_{\mathcal{L}}}{R_{y}}\right)
$$

where $\gamma, e, r_{e}, \sigma_{x, y}^{*}, I, \beta_{x, y}^{*}$ are the Lorentz factor, electron charge, classical electron radius, beam sizes at the interaction point (IP), stored beam current in the ring, and the $\beta$-function at the IP, respectively. The suffix \pm denotes each beam. The expression (10.1) is obtained from the beam-beam tune-shift parameter

$$
\xi_{ \pm x, y}=\frac{r_{e}}{2 \pi \gamma_{ \pm}} \frac{N_{\mp} \beta_{ \pm x, y}^{*}}{\sigma_{x, y}^{*}\left(\sigma_{x}^{*}+\sigma_{y}^{*}\right)} R_{x, y}
$$


Table 10.12 Progress of machine parameters of the PEP-II and KEKB B-factories

\begin{tabular}{|c|c|c|c|c|c|c|}
\hline \multirow[b]{3}{*}{ Date } & \multicolumn{2}{|c|}{ PEP-II } & \multicolumn{2}{|c|}{ KEKB (no crab) } & \multicolumn{2}{|c|}{ KEKB (crab) } \\
\hline & \multicolumn{2}{|c|}{$8 / 16 / 2006$} & \multicolumn{2}{|c|}{$11 / 15 / 2006$} & \multicolumn{2}{|c|}{$6 / 17 / 2009$} \\
\hline & LER & HER & LER & HER & LER & HER \\
\hline Circumference $[\mathrm{m}]$ & \multicolumn{2}{|l|}{2200} & \multicolumn{4}{|l|}{3016} \\
\hline Beam energy [GeV] & 3.1 & 9.0 & 3.5 & 8.0 & 3.5 & 8.0 \\
\hline Effective crossing angle [mrad] & \multicolumn{2}{|l|}{0} & \multicolumn{2}{|l|}{22} & \multicolumn{2}{|c|}{0 (crab) } \\
\hline Beam current $[\mathrm{A}]$ & 2.90 & 1.88 & 1.65 & 1.33 & 1.64 & 1.19 \\
\hline Bunches & \multicolumn{2}{|l|}{1722} & \multicolumn{2}{|l|}{1389} & \multicolumn{2}{|l|}{1584} \\
\hline Bunch current $[\mathrm{mA}]$ & 4.02 & 1.09 & 1.19 & 0.96 & 1.03 & 0.71 \\
\hline Bunch spacing $[\mathrm{m}]$ & \multicolumn{2}{|l|}{1.2} & \multicolumn{2}{|c|}{$1.8-2.4$} & \multicolumn{2}{|l|}{1.8} \\
\hline Horizontal emittance $\varepsilon_{x}[\mathrm{~nm}]$ & 30 & 50 & 18 & 24 & 18 & 24 \\
\hline RF frequency $[\mathrm{MHz}]$ & \multicolumn{2}{|l|}{476} & \multicolumn{4}{|l|}{509} \\
\hline Bunch length $\sigma_{z}[\mathrm{~mm}]$ & 10 & 10 & 8 & 6 & 8 & 6 \\
\hline$\beta_{x}^{*}[\mathrm{~cm}]$ & 30 & 30 & 59 & 56 & 120 & 120 \\
\hline$\beta_{v}^{*}[\mathrm{~cm}]$ & 0.9 & 1.1 & 0.65 & 0.59 & 0.59 & 0.59 \\
\hline Horizontal size @ IP $[\mu \mathrm{m}]$ & 95 & 158 & 103 & 116 & 147 & 170 \\
\hline Vertical size @ IP $[\mu \mathrm{m}]$ & 4.7 & 4.7 & 1.9 & 1.9 & 0.94 & 0.94 \\
\hline Beam-beam $\xi_{x}$ & 0.072 & 0.064 & 0.115 & 0.075 & 0.125 & 0.100 \\
\hline Beam-beam $\xi_{y}$ & 0.064 & 0.053 & 0.104 & 0.058 & 0.130 & 0.090 \\
\hline Luminosity $\left[\mathrm{nb}^{-1} \mathrm{~s}^{-1}\right]$ & \multicolumn{2}{|l|}{12.1} & \multicolumn{2}{|l|}{17.6} & \multicolumn{2}{|l|}{21.1} \\
\hline Integrated luminosity/day $\left[\mathrm{pb}^{-1}\right]$ & \multicolumn{2}{|l|}{858} & \multicolumn{2}{|l|}{1260} & \multicolumn{2}{|l|}{1479} \\
\hline Integrated luminosity/7 days $\left[\mathrm{fb}^{-1}\right]$ & \multicolumn{2}{|l|}{5.41} & \multicolumn{2}{|l|}{7.82} & \multicolumn{2}{|l|}{8.43} \\
\hline Integrated luminosity $/ 30$ days $\left[\mathrm{fb}^{-1}\right]$ & \multicolumn{2}{|l|}{19.8} & \multicolumn{2}{|l|}{30.2} & \multicolumn{2}{|l|}{23.0} \\
\hline Total integrated luminosity $\left[\mathrm{fb}^{-1}\right]$ & \multicolumn{2}{|l|}{557} & \multicolumn{4}{|l|}{1040} \\
\hline
\end{tabular}

The left, center, right correspond to the highest performance of PEP-II, KEKB (no crab) and KEKB (crab), respectively. The integrated luminosities are the delivered numbers for PEP-II, and recorded for KEKB. $1 \mathrm{nb}^{-1}=10^{33} \mathrm{~cm}^{-2} \mathrm{~s}^{-1}$

and the definition of luminosity

$$
\mathcal{L}=\frac{N_{+} N_{-} f}{4 \pi \sigma_{x}^{*} \sigma_{y}^{*}} R_{\mathcal{L}}
$$

where $N$ and $f$ are the number of particles per bunch and the collision frequency $(I=N e f)$, respectively, and we have assumed the beam sizes are common in two beams. The factors $R_{\mathscr{L}, x, y}$ are the geometric reduction factors due to the hour-glass effect and the crossing angle.

While a round-beam scheme may have a merit of a factor of 2 on the luminosity according to Eq. (10.1), a flat beam scheme has been chosen in most $e^{+} e^{-}$colliders, as the round-beam focusing in both planes is more difficult for an extremely small $\beta^{*}$. For a flat beam, $\sigma_{x}^{*} \gg \sigma_{y}^{*}$, the luminosity is written as

$$
\mathcal{L} \approx \frac{1}{2 e r_{e}}\left(\frac{\gamma I \xi_{y}}{\beta_{y}^{*}}\right)_{ \pm}\left(\frac{R_{\mathcal{L}}}{R_{y}}\right)
$$


Then if there is no reason to differentiate $\xi_{y}$ and $\beta_{y}^{*}$ in the two rings,

$$
\gamma_{+} I_{+}=\gamma_{-} I_{-}
$$

is resulted. As the ratio of beam energies gets larger, the boost at the collision becomes larger, but the low energy ring must store higher beam current. Thus the energy ratio was a compromise between the physics merit and the accelerator difficulty. PEP-II chose $3.1 \mathrm{GeV}$ and $9 \mathrm{GeV}$ for positrons and electrons, while KEKB chose $3.5 \mathrm{GeV}$ and $8 \mathrm{GeV}$. A larger ratio was more favored at PEP-II as it needs a magnetic separation of two beams at the IP as described later. The flavor of beams, the LER for positrons, was uniquely chosen at KEKB, where the positron acceleration for the HER was very difficult.

The actual operation of these machines, the condition (10.5) was not kept strictly, as shown in Table 10.12. One reason was that the natural size of each beam was not equal, for instance, the LER positron beam was relatively easy to be blown up due to the electron clouds at high current. Then there was a certain limit on the positron beam current and the HER current was increased beyond Eq. (10.5). This tendency was stronger in KEKB than PEP-II, as the former had stronger electron cloud effects than the latter as described later.

\subsubsection{Crossing Angle}

One of the design choices is the beam separation scheme near the IP. A crossing angle is a natural and easy solution of the separation, but the question was the experience at DORIS [125]. KEKB applied a horizontal crossing angle $2 \theta_{x}=22$ mrad, relying on simulation of the beam-beam effect. The corresponding Piwinski angle ( $\left.\equiv \theta_{x} \sigma_{z} / \sigma_{x}^{*}\right)$ was 0.86 . Their conclusion at the design stage was that the effect of the crossing angle on the beam-beam interaction would not be harmful up to their design beam-beam parameter 0.05 , if the operating betatron tunes were carefully chosen. Their choice was right and verified the vertical beam-beam parameter of 0.06 in their luminosity marching. Crossing angles were also applied at CESR and $D A \Phi N E$ colliders successfully in parallel with the KEKB operation. KEKB even prepared a crab-crossing scheme [126, 127] for the backup of the crossing angle. The ratio of the geometric reduction factors $R_{L} / R_{y}$ in Eq. (10.1) does not drastically decrease for a large crossing angle as shown in [122].

PEP-II was much more nervous on the crossing angle, then they installed a magnetic separation scheme near the IP with permanent dipole magnets [128]. This scheme also worked, but their design around the IP had to be more complicated than with a crossing angle, and gave some limitations on the performance such as the detector background due to radiative Bhabha events [129], which was much less significant in Belle. As the space at the IP was limited, they could not install a compensation system for the detector solenoid field, which might have degraded the 
beam-optical performance. Another issue of the magnetic separation was the nonnegligible effect due to the parasitic collision [130], which was never observed at KEKB.

\subsubsection{Storing High Current}

As described above, the luminosity is proportional to the stored current. To achieve the luminosity as high as $10^{34} \mathrm{~cm}^{-2} \mathrm{~s}^{-1}$, a stored current of near $3 \mathrm{~A}$ was required, which was one order higher than any high energy electron storage rings at that time. The first fundamental difficulty is to ensure the longitudinal stability of the beam.

The beam loading of the accelerating cavity is huge: a normal conducting cavity at the RF frequency $f_{\mathrm{RF}}=500 \mathrm{MHz}$ for the B-factories has a shunt impedance $R_{S} \approx 1.7 \mathrm{M} \Omega$. If the cavity is tuned at the harmonics, the $3 \mathrm{~A}$ beam generates $5.1 \mathrm{MV}$ decelerating voltage at the cavity, which is even higher than the accelerating voltage $V_{c}$ of the cavity, typically $0.5 \mathrm{MV}$. Thus the detuning of the cavity is necessary and the optimal amount of the detuning frequency is given by

$$
\Delta f=-\frac{I \sin \phi_{s}}{2 V_{c}} \frac{R_{s}}{Q} f_{\mathrm{rf}}=-\frac{P_{b} \tan \phi_{s}}{4 \pi U},
$$

where $\phi_{s}, Q, P_{b}, U$ are the synchronous phase, the $Q$-value, the beam power, and the stored energy of the cavity, respectively. If the magnitude of the detuning frequency becomes higher than or comparable to the revolution frequency, the cavity impedance hits the side bands of synchrotron motion to excite strong longitudinal coupled-bunch instabilities.

This issue of the beam-loading instability was solved in two B-factories in different ways. KEKB developed two types cavities with large stored energy, as Eq. (10.6) is inversely proportional to the stored energy. Both ARES [131] and superconducting [132] cavities could store electromagnetic energy 10 times larger than a conventional cavity. Then together with the HOM damping mechanism of them, the RF system of KEKB did not induce any beam instability up to the design current without a help of bunch-by-much feedback system. On the other hand, PEPII took a different strategy to develop a sophisticated feedback system to reduce the effective impedance seen by the beam [133]. PEP-II applied a direct RF feedback system with newly developed sideband klystrons combing a longitudinal bunchby-bunch feedback [134]. Both KEKB and PEP-II systems basically worked as expected nearly up to or even beyond their design currents.

Storing high currents caused a number of issues on the beam pipes, bellows, collimators, and even on the detectors. Direct hit of the beam of an ampere caused by beam instability or anything else easily melted down such components. The wakes at transitions resulted in discharge and heating to drive the catastrophe. A number of models have been developed and tried for the collimators, bellows, and HOM 
absorbers. Also machine protection system, loss monitors, and beam abort system had to evolve as the stored current increased.

\subsubsection{Electron Cloud}

Electron cloud was the one of the toughest issues for the asymmetric B-factories, specifically on the accumulation of the positron beam. The electron cloud had been known as a possible cause of beam instability in positive-charged beams since a long time ago such as the ISR era. Its observation [135] had been made at the Photon Factory (PF) of KEK and a theoretical explanation [136] had been done well before the start of the B-factories. What was new at the B-factories was the single-bunch instability induced by electron clouds [137]. The previous instability observed at the PF had been interpreted as a coupled-bunch instability, which was supposed to be cured by a bunch-by-bunch feedback. Thus at least KEKB was not well prepared for the single-bunch phenomena which have much higher frequency than the available feedback. Actually possibility of such a single-bunch effect had been suggested [138] before the construction of the B-factories, it had not been, however, well recognized. The single-bunch effect was experimentally confirmed at KEKB [139] as well as at CesrTA.

The electron cloud blowed up the vertical beam size drastically, and the threshold beam current was $0.4 \mathrm{~A}$ with four-bucket $(2.4 \mathrm{~m})$ bunch spacing at KEKB. The electron cloud appeared more severely in KEKB than in PEP-II, as the former had a round $\mathrm{Cu}$ beam pipe while the latter an $\mathrm{Al}$ antechamber with TiN coating. Thus the initial startup of the luminosity at KEKB was slower than PEP-II.

By applying weak magnetic field at the beam pipe, the electron cloud was removed at least in the free space. Either permanent magnets or solenoids were installed at KEKB and PEP-II to cover almost all straight sections and inside of some magnets such as quadrupoles and weak dipoles by 2004. The mitigation worked as expected and the blowup became unnoticeable at least for threebucket spacing in the case of KEKB [140]. Beside the magnetic field, various mitigation techniques have been developed and tested at the B-factories, against the formation of the electron cloud, including antechambers [141], TiN or Diamond-like carbon coatings, grooved surface pipes [142], and clearing electrodes [143]. Those techniques will be effective for future super B-factories and damping rings of linear colliders. Also several measurements of the cloud density have been carried out.

Although the density of the electron cloud became below the instability threshold by magnetic field, the betatron tune shift due to the cloud still remained in the LER at KEKB to make the tune variation along the bunch train. A mitigation for the tune variation was making use of pulsed quadrupoles as done at KEKB [144]. 


\subsubsection{Beam Optics}

The luminosity of a ring collider is inversely proportional to the vertical $\beta$-function at the IP as shown in Eq. (10.4). The B-factories have used the smallest $\beta_{y}^{*}$ as a ring collider so far. Generally speaking, a small $\beta_{y}^{*}$ means higher chromaticity and higher nonlinearity arisen from sextupoles for the chromaticity correction. Thus the design of the ring lattice needs special care to ensure the dynamic aperture. One technique applied for KEKB was non-interleaved sextupole pairs separated by a $-I$ transformation that cancel the geometric nonlinearity of the sextupoles up to the second order [145]. Although the idea was very old but the reason why the application to a real ring had to wait until the B-factories was probably the necessary computer power to optimize the sextupole setting, as it requires a large number of sextupole families to extend the momentum acceptance. For instance, KEKB had 54 families of sextupole pairs. The relative betatron phase advance between the pairs became adequate by using the $2.5 \pi$ cell structure in the case of KEKB arc section [146].

Another technique to enlarge the dynamic aperture was to place a special chromaticity correction section near the IP. The beam optics became somewhat similar to that for linear colliders in this case. KEKB designed such a section for the vertical correction, while PEP-II horizontal for their LERs.

These schemes worked as expected for the B-factories, and expected to work for future super B-factories and light sources. Once the chromaticity correction is solved, the next source of the nonlinearity is the fringe field of the final quadrupole and the geometric nonlinearity at the IP [147], which may be mitigated by additional octupoles placed at the final quadrupoles.

The $x-y$ coupling and the residual vertical dispersion all over the ring were one of the keys to achieve a high luminosity by reducing the vertical emittance. Various techniques have been applied for such optics measurements and corrections [148-150]. A counter solenoid to the detector solenoid was also effective to reduce the coupling source in the case of KEKB. This was also important in the case of crab crossing where the luminosity performance was sensitive to the chromatic $x-y$ coupling as described later.

\subsubsection{Beam Diagnostics and Control}

A number of beam diagnostic methods were developed and applied for the Bfactories:

- Beam position monitors (BPMs) with a resolution better than $1 \mu \mathrm{m}$ in the average mode. In some cases turn-by-turn or bunch-by-bunch electronics were equipped [151]. In the case of KEKB, the gain imbalance of the electrodes through the electronics was calibrated using a beam-mapping technique [152]. The design 
of the electrodes and the electronics were carefully done for the high-current operation.

- Beam-based alignment of BPMs was regularly carried out. Displacements of BPMs near sextupoles, caused by heating from the stored current, were monitored at KEKB [153].

- Bunch-by-bunch feedback systems were installed both in PEP-II and KEKB. Only PEP-II had the longitudinal system to suppress the beam-loading instability as described above. A collaboration including the DA $\Phi N E$ team has been developed on the system for the present and future applications [154].

- Betatron tune monitor: controlling the betatron tune was extremely important to maximize the luminosity. The basic idea at KEKB was to monitor the tunes of pilot bunches in each ring that did not collide to the other beam. Tune feedback with these bunches was also applied to control them within an accuracy of $\Delta v \approx 10^{-4}$.

- Synchrotron radiation beam profile monitors. For the visible light, an interferometer was used especially for the vertical size measurement [155]. Special gated cameras were also used to observe the beam size of individual bunch, esp. to diagnose the electron-cloud effects [156].

- Beam loss monitors and beam abort system: both machines were very nervous to protect the machine against accidental beam losses caused by instabilities, RF trips, wrong injection, or whatever. The most sensitive and expensive loss monitor was the BaBar and Belle detectors, which generated beam abort signals if necessary. A number of beam loss monitors such as ionization chambers and PiN diodes were distributed around the ring, esp. near the collimators. The beam abort system consists of an abort kicker and a beam damp. The abort kicker had a rise time of $0.5 \mu \mathrm{s}$ in the case of KEKB.

- The injectors had developed their own diagnostics including BPMs, wire scanners, streak cameras, etc.

- All accelerator components were controlled by computer control systems either by EPICS at KEKB [157] or a legacy system at PEP-II. An online modeling such as SAD for KEKB [149] was also important to achieve the luminosity.

\subsubsection{Collision Tuning}

Starting up the colliders after a period of long shut down, the following procedures were necessary to recover the luminosity:

- Global coupling/dispersion/ $\beta$-function correction all over the ring. The global orbit was then locked to the "golden" orbit that was resulted by the optics correction.

- Locking the betatron tunes of the pilot bunches.

- The beam steering at the IP looking at the beam-beam deflection. 
- In the case of the crossing angle at KEKB, the horizontal offset at the IP was controlled by looking at the vertical beam size measured by the interferometer [158].

- Tuning of the local coupling and dispersion at the IP by making offsets of orbits at sextupoles near the IP [149].

- Dithering technique was used at PEP-II to maximize the luminosity against the beam offsets [159].

- Skew sextupoles were introduced at KEKB to correct the local chromatic $x-y$ coupling terms at the IP [124].

The horizontal tunes were chosen as close to a half integer as possible, to maximize the luminosity using the dynamic- $\beta$ effect and expecting the reduction of the degree-of-freedom of the beam-beam interaction [160]. In the case of KEKB, the LER and the HER was operated at $v_{x} \approx 0.506$ and $v_{x} \approx 0.510$, respectively. Both the optics correction and the tune feedback were necessary to maintain a collision near the stop band.

\subsubsection{Injector}

The electron-positron injector must provide enough number of charges to the collider rings. PEP-II could fully utilized the injection system for SLC, which had more than enough performance for PEP-II, in the intensity, repetition, and the emittance, especially with the damping rings for both beams. On the other hand, the injector for KEKB was upgraded from that for TRISTAN, having only the minimum performance to satisfy the requirements of the injection to KEKB as shown in Table 10.13. In early days, it was thought that the performances of the two machines would be eventually limited by the performance of each injector. Actually such a situation did not happen. The key was the top-up operation applied for the both machines since 2004. Then the necessary strength of the injected beam became much smaller than the maximum performance even at KEKB [161]. Both machines were the first to have utilized the top-up for high-energy colliders, even earlier than the most of light sources. The 2-bunch per pulse acceleration and installing a Cband section in the linac [162] also contributed to make the gap between PEP-II

Table 10.13 Comparison of positron injection

\begin{tabular}{l|l|l|l}
\hline & \multicolumn{2}{|l|}{ KEKB } & \multicolumn{1}{l}{ PEP-II } \\
\cline { 2 - 4 } & \multicolumn{2}{|l}{1999} & \multicolumn{2}{l}{2010} \\
\hline Production energy $[\mathrm{GeV}]$ & 4 & 30 \\
\hline Particles per pulse $\left[10^{10}\right]$ & 0.4 & 1.0 & 2 \\
\hline Repetition rate $[\mathrm{Hz}]$ & 50 & $\leq 120$ \\
\hline Invariant emittances H/V $[\mu \mathrm{m}]$ & $\sim 3000 / 3000$ & $3 / 0.3$ \\
\hline $\mathrm{e}^{+} / \mathrm{e}^{-}$switching time $[\mathrm{s}]$ & 300 & 0.02 & 0 (simultaneous) \\
\hline
\end{tabular}


and KEKB smaller. KEKB has solved the conflict with the injection to the light sources by introducing a pulse-to-pulse switching of the linac.

\subsubsection{Crab Crossing}

KEKB operated with crab crossing from 2007 through 2010 using superconducting crab cavities [163] installed one cavity per ring [164]. KEKB had already achieved a luminosity $1.76 \times 10^{34} \mathrm{~cm}^{-2} \mathrm{~s}^{-1}$ by then with the crossing angle, so the intension of the crab crossing was not simply the backup. Simulations of beam-beam effect indicated that a head-on or crab collision could increase the beam-beam parameter $\xi_{y}$ even higher than 0.15 combining with a horizontal betatron tune close to a half integer [165]. Thus the hope was to experimentally verify the possibility of such a high beam-beam parameter, considering super B-factories.

The crab cavities were successfully installed and operated for more than 3 years. The luminosity was actually increased as shown in Table 10.12. The resulted beambeam parameter was 0.09 , which was indeed higher than the value with crossing angle, but much less than the simulation (0.15). It was not easy to single out the cause, but there were indications that remaining higher-order terms of the beam optics at the IP degraded the luminosity and $\xi_{y}$. One example was the chromatic $x-y$ coupling terms at the IP [166]. By installing skew sextupoles in the arc as the tuning knobs, the luminosity was improved by up to $10 \%$ [124]. Then a speculation was that any higher order terms at the IP could degrade the performance. It was not easy to estimate how many terms were relevant and how to correct them, as there were almost no direct beam diagnostics at the IP except the luminosity. What was verified at KEKB was that the crab crossing itself should work for any colliders up to $\xi_{x, y} \lesssim 0.1$, including the Large Hadron Collider (LHC).

\subsubsection{SuperKEKB}

Although the crab crossing scheme tested at KEKB achieved then-highest luminosity, it also showed the difficulty to realize the very high beam-beam parameter $\gg 0.1$. Thus the direction toward a higher luminosity asymmetric collider must change. An alternative idea, Nano-beam scheme, developed by P. Raimondi [167] saved the next generation of KEKB. The idea by Raimondi consists of:

- A large crossing angle, in terms of Piwinski angle $\theta_{x} \sigma_{z} / \sigma_{x}^{*} \gg 1$, where $\theta_{x}$ is the half horizontal crossing angle.

- A very short $\beta_{y}^{*} \sim \sigma_{x}^{*} / \theta_{x} \ll \sigma_{z}$.

- Small horizontal/vertical emittances. 
Fig. 10.13 Beam crossing in the nano-beam scheme



The formula for the luminosity, Eq. (10.1) is still valid in the nano-beam scheme. The major gain of the luminosity comes from a very short $\beta_{y}^{*}$. Unlike a head-on scheme, the bunches intersect one another only within $\sigma_{x}^{*} / \theta_{x}$ around the IP due to the large Piwinski angle as shown in Fig. 10.13.

As the length of the intersecting region is $\sigma_{x}^{*} / \theta_{x} \ll \sigma_{z}$, the condition to avoid an hour-glass effect becomes $\beta_{y}^{*} \lesssim \sigma_{x}^{*} / \theta_{x} \ll \sigma_{z}$. In the case of SuperKEKB, it is possible to choose $\beta_{y}^{*} \sim 0.3 \mathrm{~mm}$, which gives $\times 20$ gain compared to KEKB. Then the bunch length $\sigma_{z}$ is not necessary to be very short, thus it is possible to avoid unfavorable effects such as the coherent synchrotron radiation. This scheme does not require a very high $\xi_{y}$, so $\xi_{y} \sim 0.09$, which has been achieved at KEKB, is assumed at SuperKEKB. The crossing angle itself can be larger than the previous machines, since it does not need crab crossing any more. As it does not require an operation very close to a half-integer tune, and also the horizontal beam-beam parameter is very small, the dynamic emittance effect is not an issue. The situation of the nano-beam crossing is similar to a collision with many micro-bunches which have a short bunch length $\sigma_{x}^{*} / \theta_{x} \ll \sigma_{z}$.

Actually Raimondi has proposed one more important idea, crabbed waist scheme, on top of the nano-beam scheme [167]. The crabbed waist aligns the vertical waist along the center line of the other beam, to reduce the dependence of the beam-beam effect on the horizontal displacement of a particle. This scheme should improve $\xi_{y}$ by reducing the synchrotron-betatron coupling caused by the crossing angle. Although the crabbed waist scheme has merits on the collision itself, its realization needs further study. A simple way to introduce the crabbed waist is to install a pair of sextupoles in both sides of the IP. The nonlinearities of these sextupoles can be canceled by $-I$ or $I$ transformation between the pair, but the unavoidable nonlinearities around the IP interfere the cancellation to reduce the dynamic aperture drastically in the case of SuperKEKB. Such nonlinearities include the fringe field of the final quadrupoles, geometric nonlinearities at the IP, and nonlinear fields in the quadrupoles and solenoids. As these terms increase for smaller $\beta_{y}^{*}$, the solution may be non-trivial and has not been found at least for SuperKEKB yet. Thus the crabbed waist scheme is not included in the base line design at SuperKEKB.

SuperKEKB started the beam operation in early 2016. The commissioning has been carried out in three phases: no collision (Phase 1, February-June 2016), collision without central vertex detector of Belle II (Phase 2, March-July 2018) [168], and collision with full Belle II (Phase 3, March 2019-). The design parameters [169] 
Table 10.14 Parameters of SuperKEKB, design and typical values in phase 2, comparing to $\mathrm{KEKB}$ with crab crossing, where the effective crossing angle is zero

\begin{tabular}{|c|c|c|c|c|c|c|c|}
\hline \multirow[b]{3}{*}{ Date } & \multicolumn{2}{|c|}{ SuperKEKB } & \multirow{2}{*}{\multicolumn{2}{|c|}{\begin{tabular}{|l} 
SuperKEKB \\
June 2018
\end{tabular}}} & \multicolumn{2}{|c|}{ KEKB (crab) } & \\
\hline & \multicolumn{2}{|l|}{ Design } & & & \multicolumn{2}{|c|}{ June 17, 2009} & \\
\hline & LER & HER & LER & HER & LER & HER & \\
\hline Circumference & \multicolumn{6}{|l|}{3016} & $\mathrm{~m}$ \\
\hline Beam energy & 4.0 & 7.0 & 4.0 & 7.0 & 3.5 & 8.0 & $\mathrm{GeV}$ \\
\hline Crossing angle & \multicolumn{4}{|l|}{83} & \multicolumn{2}{|c|}{$22 / 0$ (crab) } & $\mathrm{mrad}$ \\
\hline Beam current & 3.8 & 2.6 & 0.27 & 0.225 & 1.64 & 1.19 & A \\
\hline Bunches & \multicolumn{2}{|l|}{2500} & \multicolumn{2}{|l|}{395} & \multicolumn{2}{|l|}{1584} & \\
\hline Bunch current & 1.5 & 1.0 & 0.67 & 0.55 & 1.03 & 0.71 & $\mathrm{~mA}$ \\
\hline Bunch spacing & \multicolumn{2}{|l|}{1.2} & \multicolumn{2}{|l|}{$\sim 7.2$} & \multicolumn{2}{|l|}{1.8} & $\mathrm{~m}$ \\
\hline Hor. emittance & 3.2 & 4.6 & 1.8 & 4.6 & 18 & 24 & $\mathrm{~nm}$ \\
\hline RF frequency & \multicolumn{6}{|l|}{509} & $\mathrm{MHz}$ \\
\hline Bunch length $\sigma_{z}$ & 6 & 5 & 4.6 & 5.3 & 8 & 6 & $\mathrm{~mm}$ \\
\hline$\beta_{x}^{*}$ & 3.2 & 2.5 & 20 & 10 & 120 & 120 & $\mathrm{~cm}$ \\
\hline$\beta_{y}^{*}$ & 0.27 & 0.3 & 3 & 3 & 5.9 & 5.9 & $\mathrm{~mm}$ \\
\hline Hor. size @ IP & 10 & 11 & 19 & 21 & 147 & 170 & $\mu \mathrm{m}$ \\
\hline Ver. size @ IP & 0.048 & 0.048 & 0.56 & 0.56 & 0.94 & 0.94 & $\mu \mathrm{m}$ \\
\hline Piwinski Angle & 24.9 & 18.9 & 10.0 & 10.5 & $(0.60)$ & $(0.39)$ & \\
\hline$\sigma_{x}^{*} / \theta_{x}$ & 0.24 & 0.27 & 0.46 & 0.51 & $(13.3)$ & (15.4) & $\mathrm{mm}$ \\
\hline Beam-beam $\xi_{x}$ & 0.0028 & 0.0012 & & & 0.125 & 0.100 & \\
\hline Beam-beam $\xi_{y}$ & 0.088 & 0.081 & 0.030 & 0.021 & 0.130 & 0.090 & \\
\hline Luminosity & \multicolumn{2}{|l|}{800} & \multicolumn{2}{|l|}{2.3} & \multicolumn{2}{|l|}{21.1} & $/ \mathrm{nb} / \mathrm{s}$ \\
\hline
\end{tabular}

$1 / \mathrm{nb}=10^{33} \mathrm{~cm}^{-2} \mathrm{~s}^{-1}$

and a typical performance of Phase 2 operation [170] of SuperKEKB are listed in Table 10.14 .

The commissioning in Phase 2 has achieved several milestones of the project:

- Verified the collision with nano beam scheme with Piwinski angle $\sim 10$. Although the luminosity was still much less than the design or achieved at KEKB, no fundamental limitations are found. The highest luminosity $56 / \mathrm{nb} / \mathrm{s}$ was recorded during Phase 2 [170]. An experimental verification of coherent beam-beam instability [171] also assures the understanding of the beam-beam effect.

- The upgrade of the injector several new components: RF gun, positron target, and positron damping ring was basically successful [172].

- The mitigation of e-cloud in the LER has well suppressed the blowup of the vertical beam size up to $1 \mathrm{~A}$ of the stored beam [173].

The key toward the design luminosity is higher stored current with smaller $\beta * \mathrm{~s}$. Both the stored currents and $\beta^{*}$ s are still far from the design. Table 10.14 shows that the achieved $\beta_{y}^{*}$ is much longer than the length of the interaction area, $\sigma_{x}^{*} / \theta_{x}$. It means that the merit of nano-beam scheme has not been obtained yet. One possible obstacle for higher current and smaller $\beta^{*} \mathrm{~s}$ is the robustness of superconducting 
final focus quadrupoles [174] against quenches due to beam losses. Quenches of those magnets have been seen in the Phase 2 commissioning both for the stored and injected beams.

\subsection{Tevatron-HERA-LHC}

Karl-Hubert Mess · Peter Schmüser

\subsubsection{Three Steps in the Evolution of Superconducting Accelerator Magnets}

The first particle accelerator approaching the TeV energy range was the protonantiproton collider Tevatron at the Fermi National Accelerator Laboratory. Much pioneering work was done at Fermilab, and many of the successful design and construction principles of the Tevatron dipole and quadrupole magnets have been adopted at the electron-proton collider HERA and the Large Hadron Collider LHC (CERN). The superconducting coils used in these accelerators show great similarities. They are wound with high precision from a multistrand cable containing many thousand fine niobium-titanium filaments in a copper matrix. The coils have a helium-transparent insulation and are confined by nonmagnetic clamps (often called collars). The clamps are assembled from precision-stamped stainless-steel or aluminum-alloy laminations and serve three purposes: they define the exact coil geometry, they exert a large prestress on the coils to prevent conductor motion during excitation of the magnet, and they take up the huge magnetic forces at large fields. Interesting differences, however, have evolved in the layout of the iron yoke. The Tevatron magnets are "warm-iron" magnets with a yoke at room temperature while the HERA and LHC magnets feature a "cold-iron" yoke inside the liquid helium cryostat.

In the beginning of the HERA project two different design lines were followed. Our group at DESY constructed and built $6 \mathrm{~m}$ long prototype dipoles of the "warmiron" type which were basically copies of the Tevatron dipoles. These magnets were made with tooling suitable for series production and performed very well, the design field of 5.2 T was exceeded and excellent field quality was achieved. In parallel, an industrial company (Brown Bovery in Mannheim) designed and built two "cold-iron" prototype dipoles following a concept developed at Brookhaven National Laboratory. Here the coil was surrounded with epoxy-fibreglass form pieces and then directly mounted in an iron yoke which served as the clamping structure. Also the BBC dipoles showed remarkable performance. They exceeded the design field by an ample margin, however the field quality became poor for 
fields above $4 \mathrm{~T}$ owing to saturation effects in the nearby iron yoke. ${ }^{2}$ With the aim in mind of combining the virtues of both design lines, while avoiding the relative drawbacks, the idea was conceived to take the well-proven aluminumcollared coil of the first design line and put an iron yoke immediately around the collars. Our main motivation was magnet safety in case on a quench (breakdown of superconductivity).

Quench protection is an important task in any large superconducting (sc) magnet system. Accelerator magnets have slim coils with a small copper-to-superconductor ratio. They are definitely not cryostable (cryostability means that the copper in the cable is able to carry the full current). If a HERA dipole quenches it is mandatory to ramp down the current of 5000-6000 A with a time constant of less than a second to prevent overheating and possible destruction of the coil. The protection of a single magnet is straightforward: if a quench is detected the coil is connected to a dump resistor via a thyristor switch and the current decays exponentially $I(t)=I_{0} \exp (-t / \tau)$. The stored magnetic energy is dissipated in the dump resistor.

In an accelerator this simple solution is not possible since the magnets in a long string are connected in series. In HERA this string is a $45^{\circ}$ arc comprising 52 dipoles and 26 quadrupoles. Current leads from the sc coils to the room-temperature environment are only installed at the ends of the string but not at individual magnets. Owing to the large inductivity of the 54-magnet string a long decay time constant $\tau \approx 20 \mathrm{~s}$ must be chosen in order to limit the induced voltage against ground potential to less than $1000 \mathrm{~V}$. The quenched coil would burn up during such a slow current decay, hence an electric bypass must be provided. Here the great advantage of a cold iron yoke comes in: it is easy to provide such a bypass by mounting a superconducting cable, reinforced with a copper bus bar, in a groove at the outer rim of the yoke. The bypass conductor is connected to the main current conductor via a "cold" silicon diode inside the liquid helium cryostat. ${ }^{3}$ The diode has a threshold voltage of about $1 \mathrm{~V}$ at liquid helium temperature, hence the current flow through the bypass is zero as long as the magnet coil is in the superconducting state (during particle accceleration the ramp speed must be chosen so low that the inductive voltage stays well below the $1 \mathrm{~V}$ threshold). However, a quenched coil develops rapidly a resistive voltage exceeding $1 \mathrm{~V}$, and then the main current switches automatically over to the bypass. The Tevatron possesses an active quench protection system which is more complicated and shall not be discussed here.

Potentially dangerous are quenches at localized spots in the coil. If the normal zone does not propagate fast enough along the coil it may happen that the stored magnetic energy is dissipated in the vicinity of the quench origin leading to local overheating. To avoid this, so-called "quench-heaters" are installed which are electrically heated when a quench has been detected. Their task is to spread the normal zone over the entire coil. Figure 10.14 shows that a warm-iron magnet may

\footnotetext{
${ }^{2}$ It has found out later in the LHC and RHIC projects that the detrimental effects of iron saturation can be alleviated by punching a suitable hole pattern into the iron yoke laminations.

${ }^{3}$ The cold diode concept was invented and thoroughly investigated at BNL.
} 


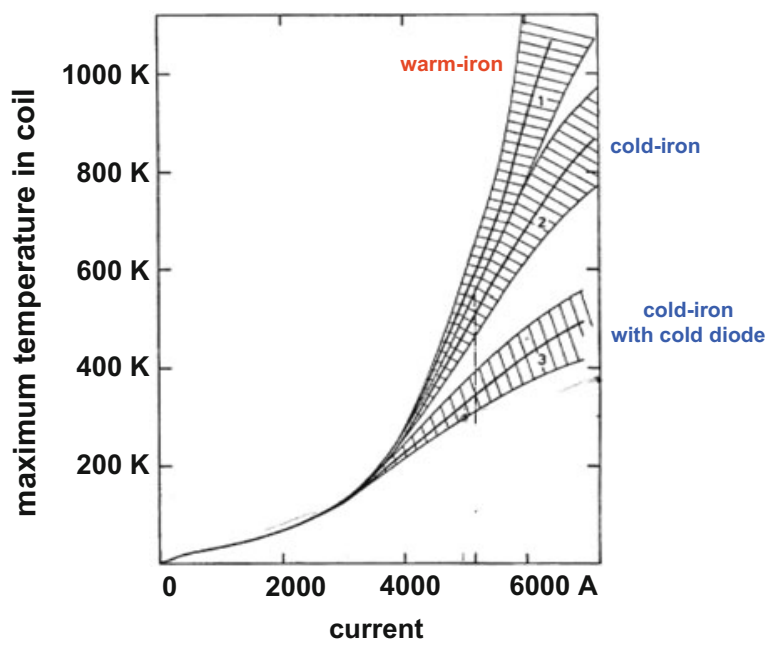

Fig. 10.14 Simulation of temperature rise in a dipole after a quench at a localized spot. Plotted is the maximum temperature in the coil as a function of coil current. It is assumed that the quench heaters fail and that the current is ramped down exponentially with a time constant of $17 \mathrm{~s}$. Three magnets types are considered: the warm-iron HERA prototype dipole, the cold-iron BBC dipole without a cold diode, and the cold-iron BBC dipole with a cold diode. In the first two cases the bypass is provided by two curent leads from the superconducting cable in the liquid helium container to the room-temperature environment, a normal-conducting cable and a thyristor switch outside the cryostat. Adapted from a DESY Internal Note January 1984 by K.H. Mess and P. Schmüser

heat up to quite dangerous temperatures if the quench heaters fail, while the coldiron magnet equipped with a cold diode and a superconducting bypass remains in a safe temperature regime.

The HERA-type magnet offers several more advantages. The cryostat can be a simple steel vessel which is easier to fabricate and less expensive than the very slim stainless-steel cryostat needed in the warm-iron magnet. The coil must be accurately centered with respect to the yoke to avoid field distortions and asymmetry forces. In the HERA design a precise centering is achieved by a tongue-groove combination in the collar and yoke laminations. In contrast to this, coil centering in a warm iron yoke requires many adjustable supports which constitute a considerable heat load on the cryogenic system.

The successful HERA magnet design has had a considerable impact on the layout of the LHC magnets. ${ }^{4}$ The elegant twin-aperture magnet can be realized with this concept. The two counterrotating proton (or heavy ion) beams are guided and focused by two nearby sc coils of opposite polarity which are mounted in a common iron yoke. This yoke can be made rather slim since most the magnetic flux

\footnotetext{
${ }^{4}$ The evolution of the twin-aperture LHC magnets is described in an article by Lucio Rossi, CERN Courier October 2011.
} 
of one coil returns through the aperture of the neigbouring coil and not through the iron. Another novel feature of the LHC magnets is the cooling by superfluid helium which extends the achievable field into the $9 \mathrm{~T}$ regime. A significant cost reduction is achieved by the fact that only one cryostat system is needed for both particle beams. The compactness of the magnets was very important for the installation in the tunnel, it would have been extremely demanding if not impossible to mount two separate proton storage rings in the narrow LEP tunnel.

The elegance and cost-effectiveness of LHC becomes obvious in comparison with the Superconducting Super Collider SSC project which was promoted in the USA in the 1980s. Here two counterrotating $20 \mathrm{TeV}$ proton beams were planned to be accelerated and stored in two separate rings equipped with magnets of an improved Tevatron design. This "conservative" approach turned out far more costly than the innovative LHC solution. The SSC was cancelled in 1993 for budget reasons.

\subsubsection{HERA Experience and the Design of Future Lepton-Hadron Colliders}

The scientific results from HERA on quantum chromo-dynamics (QCD) are an essential pillar of our present understanding of the nature of strong interactions. These insights are of critical importance for the interpretation of the measurements of particle production processes at the Large Hadron Collider (LHC) at CERN. There are, however, very interesting phenomena and physics questions that were raised by the HERA experiments but remain unanswered as of today:

- How does QCD lead to the rich and complex structure we observe in nuclei?

- How are quarks and gluons and their spins distributed in nucleons and nuclei?

- Why do protons and neutrons have spin $1 / 2$ ?

- How does the increase of gluon density with decreasing gluon fractional momentum x saturate?

- What is the distribution of partons that seed the new form of matter discovered at RHIC, the quark-gluon plasma?

The U.S. Nuclear physics community has formulated a physics program that addresses these questions in a White Paper [175]. This physics program is based on an Electron Ion Collider (EIC), which can provide collisions between polarized electrons and ions ranging from polarized proton to Uranium over a wide range of center of mass energies $29-140 \mathrm{GeV}$ with a high luminosity in the order of $10^{34} \mathrm{~cm}^{-2} \mathrm{~s}^{-1}$. There are two designs for an EIC under development in the U.S., one by Thomas Jefferson National Accelerator Facility, called JLEIC [176], and one by Brookhaven National Laboratory, called eRHIC [177]. Both designs are based on intersecting storage rings. 
The experience gained at HERA with high luminosity lepton proton collisions, beam dynamics, various enabling technologies, the electron storage ring spin dynamics, and the control of collective effects have a tremendous impact on the design of these machines, in particular, the layout of the interaction regions.

HERA beam dynamics performance is used to benchmark computer codes needed to develop the EIC designs. HERA operational experience is extremely important for designing the complex interaction regions with unprecedented detector acceptance, assessing detector backgrounds induced by the beams and their mitigation. The design of the magnets which were developed for the HERA interaction regions have been carried over for the design of other colliders, such as BEPC-II, KEKB, ILC, LHeC, and eRHIC. Some beam dynamic phenomena observed in HERA directed the attention of the EIC designers to specific aspects of electron ion collisions.

The examples in the following sections illustrate the positive impact of the HERA design and operational experience on other colliders and in particular to the Electron Ion collider eRHIC.

\subsubsection{Lepton-Hadron Beam-Beam Interactions}

The concept of lepton proton collisions in HERA was to adjust the the strength of the beam-beam force for each beam such as this beam would collide with a beam of its own species. A typical beam-beam strength in terms of incoherent beam-beam tuneshift for $e^{+}-e^{-}$collisions was $x_{x, y}^{e} \simeq 0.03$. For Hadron, the strengths of beam beam interactions was typically below $x_{x, y}^{p} \simeq 0.01$. This succesful concept was used successfully in other colliders such as KEKB and is also adapted at the EIC design.

Occasionally, when the HERA beams were brought into collisions. an observation was made which is a concern for the electron ion collider. Under the influence of strong beam-beam interaction, the electron beam can develop coherent transverse oscillations. If this happens, the unstable motion of the electrons affects in turn the hadron beam via the beam-beam coupling. The hadrons will start to oscillate as well driven by the beam beam field of the unstable electron beam and the hadron beam emittance will then filament in phase space and become diluted that way. The effective beam size grows considerably and practically irreversely in the process which quickly renders the beam unusable.

This phenomenon was observed only occasionally at HERA and its rare occurrence made it impossible to perform systematic studies. Furthermore the impact on operations was very small and therefore, no resources have been invested to study the effect in detail.

The experience with lepton proton collisions in HERA, raised a concern that this effect would occur regularly in the EIC, because of significantly enhanced beam-beam parameters per collision in the EIC. This triggered realistic beam-beam interaction simulations for eRHIC. The beam-beam study was performed using the 
so-called strong, strong model, where each beam is described by a large number of super-particles and the thin lens beam-beam lenses are split into many beambeam-lens slices. The individual particle's trajectories are impacted by the collective electro-magnetic forces induced by the particles of the opposite beam, respectively. This consitutes a fully dynamical model of the beam-beam interactions. The result of this study was that this effect indeed exists and would affect the performance of the EIC if not avoided by a careful choice of the machine parameters. The threshold in proton bunch intensity for this catastrophic instability is a factor of two above the chosen eRHIC design values of proton bunch intensity [177].

\subsubsection{Beam-Gas Backgrounds of the Colliding Beam Detectors}

The experience with beam induced backgrounds at HERA is very important for the design of the interaction regions and the collision detectors of the EIC or LHeC.

The combination of synchrotron radiation emitted by the electron beam in the separator- and the focusing fields when entering the interaction region and the corresponding strong sources of desorbed gases in the vicinity of the collision detectors caused initially strong hadron-beam-gas induced detector backgounds.

These backgrounds improved slowly by conditioning the surfaces of masks and absorbers by the presence of electron beam and scattered synchroton photons. Eventually a very high dynamical vacuum quality in the order of 1 nbar with full beam intensity was achieved. This required a installation of a large pumping speed and good vacuum conductance with integrated NEG pump in the IR quadrupole magnets and Titanium supplimation pumps. The cold beam pipe of the superconducting IR magnets (at a temperature of $80 \mathrm{~K}$ ) acted as a cryopump and helped to improve the vacuum. But once the surface was saturated, the magnes needed to be warmed up and the accumulated gas gas molecules on the cold surfaces needed to be removed from the IR vacuum system using turbo pumps. Each warm-up and cooldown cycle marked a step in background improvement.

Another important lesson learned from HERA IR operations was that the vacuum system must be designed such that IR vacuum leaks are unlikely and that venting of the IR and detector vacuum for maintenance and repair purposes must be avoided by design.

The analysis of HERA backgrounds [178] shows the importance of extremely good vacuum quality in the beam vacuum chamber inside the central colliding beam detector. This makes high vacuum pumping speed and high vacuum conductance within the detector beam pipe mandatory. Techniques such as in situ bakeout and NEG coating of the detector beam chamber might be unavoidable to overcome this difficulty.

This experience is exploited in the design of the interaction regions of the EIC, in particular at eRHIC. First background simulations indicate that difficult initial background conditions as experienced in HERA can be avoided taking the lessons learned into account. 


\subsubsection{Hadron Beam Collimation}

In lepton-hadron collisions with stong beam-beam interaction, the hadron beam will develop tails in the tranverse particle distribution. The controlled removal of these halo-particles is very important for low detector background and efficient data taking.

A sophisticated collimation system and collimator optimization scheme was implemented in HERA. It consisted of two stages of collimation, with one single primary collimator per oscillation plane and two secondary collimators per plane. A detailed and fully automated collimator adjustment procedure was developed which provided in general good background conditions routinely.

The HERA collimation system and its operational optimization techniques are being carried over to the eRHIC design version of the EIC.

Furthermore, high energy proton operation with the HERA-B [179] fixed target in HERA, revealed that the wire target could be integrated in the existing collimator system. It consitituted an excellent beam-edge spoiler target which significantly increased the efficiency of the two stage collimation system and led to a significant reduction of particle background in the detectors H1 and ZEUS.

Early simulations of halo particle backgrounds in HERA showed clearly, that the halo must be removed far away from the detectors and local shields or masks will only aggravate the background conditions for the colliding beam detectors [180].

Experience with HERA collimation system have been taken into account in the elaborated LHC collimation system and are an important input for the design of the collimation system of eRHIC.

\subsubsection{Spin Polarization of the HERA Electron Beam}

The good performance of the HERA electron spin in colliding beam operations at the North and the South interaction points with three pairs of spin rotators in the North, South, and East straight sections which provided a polarization of $\geq 50 \%$ [181] was vital for the HERMES experiment [182] and augmented the physics program of the H1 and ZEUS detectors [183]. This performance was achieved with uncompensated detector solenoids in the North (H1), South (ZEUS) interaction reagions. The spin tuning procedures developed for HERA which included the system of harmonic bumps, and spin-matching of the straigth sections between the rotator pairs, as well as the electron beam working point near the integer resonance were important factors in this success.

The HERA experience constitutes an important reference point for designing the EIC for high electron spin polarization. Spin physics is an important part of the EIC physics program. Collisions between highly polarized electron and Hadron beams with all combination of spin helicities enable an this physics program.

The spin matching techniques and the scheme of orbit optimization with harmonic bumps which contributed to the success of the HERA spin program, are being carried over to the eRHIC design to enable operation with highly polarized 
electron beams. As in HERA, an equilibrium polarization of at least $50 \%$ has been shown to be achievable in eRHIC at the highest electron beam operation energy of $18 \mathrm{GeV}$. This performance will enable to maintain a high level of average polarization of $\simeq 80 \%$ with the initial polization of the the electron beam of $85 \%$ if the stored electron bunches are replaced every 6 min on average [177].

\subsubsection{Lessons Learned from HERA Dynamic Aperture}

The dynamic aperture optimization in the HERA electron ring after the year 2000 was accomplished with only two sextupole families for the correction of chromaticity. Correction of higher order chromaticity which is due to the strong contribution of the interaction region quadrupole magnets to the chromaticity and the corresponding strong off-momentum distortion of the optical functions (betabeat) is necessary to confine the beam tune foot print and to avoid destabilizing resonances.

The straight forward correction of off-momentum beta-beats with more families of sextupoles was avoided thereby avoiding large peak strengths of the sextupole magnets, which would have deteriorated the dynamic aperture. Instead, the horizontal and vertical betatron phase differences between the North and the South interaction points were chosen to be an odd integer of $\pi / 2$. The off-momentum beta beat caused by the low beta quadrupoles of one IR was intrinsically canceled by the correponding beta beat of the other IR. This way, the nonlinear tuneshift with momentum was minimized.

The HERA chromatic correction scheme [184] also suppressed the generation of driving terms of the higher order nonlinear synchro-betatron resonances $Q_{x}+3 \cot Q_{s}=$ integer which otherwise would have affected the beam stability at the low tune values needed for high polarization operation.

Last but not least, the HERA chromatic correction scheme reduced the magnet currents of the sextupoles circuits which would have been required using the multifamily scheme. The HERA scheme is being carried over to eRHIC which is planned to be operated with two collidng beam detectors as well. The eRHIC chromatic correction scheme is an important constituent of the design which enables highest luminosity values.

\subsubsection{HERA IR Magnet Design}

In order to achieve hightest luminosity, the HERA interaction regions have been re-designed to allow for very small beta functions at the IP. The change was implemented in the year 2000 and HERA operated with about 3.5 times the design luminosity in the years 2004-2007.

This upgrade included accelerator magnets that were placed inside the colliding beam detectors $\mathrm{H} 1$ and ZEUS, and in particular inside the solenoidal detector fields of up to $5 \mathrm{~T}$. Obviously, no magnetic steel was allowable inside the solenoid fields 
of the detectors. Furthermore, the accelerator magnets should have minimum mass to minimize the distorions of the trajectories of scattered particles, maximizong the detector acceptance this way and avoiding introduction of strong scattering targets insode the detector. Thus superconducting technology without steel collaring needed to be used. The available space for these magnets was very limited, as the detectors already existed and could not be modified. The magnets needed to be combinded function to separate the lepton and the hadron beam and to start focusing the electron beam as early as possible. The aperture of the magnet needed to be very large to allow the synchrotron beam to pass through the entire detector and lowbeta quadrupole section. Furthermore, the aperture needed to provide space for the envelopes of the separated beams.

To meet these requirements, a new type of superconducting magnet was developed by a collaboration of scientists from Brookhaven National Laboratory and DESY [185]. The supercnducting magnet coil was produced by developing the 2D direct wind method used for the RHIC corrector magnets to 3-D geometry such that the superconducting wire could be attached directly to the surface of a cylinder. The magnet coil was wound directly on the beam pipe using a seven-strand NbTi superconducting cable and the coil was stabilized with glass-fiber tape, a technique carried over from the HERA multipole corrector coils.

High field quality of superconducting magnets requires high precision in the manufacturing of the coil. For the HERA interaction region magnets, the precision was achieved by combining the RHIC and HERA corrector coil technologies.

The coil was wound on a precision-machined cylinder which was coated with epoxy. A computer controlled stylus placed superconducting wire with high precision on the cylinder. It also transmitted ultra-sound waves to the wire, which by means of friction melted the epoxy surface of the cylinder thereby fixing the superconducting wire in its position. After winding the first layer of the coil, it was complemented with GF spacers and fixed with glass-fiber tape under high tension before the coil was vacuum impregnated with epoxy. The field of the first layer was measured and compared with the calculated field for perfect wire position. Discrepancies were corrected by modifying the definition of the second layer wire positions correspondingly. After high precision machining the new surface, the magnet was ready for the second layer of coil to be wound. This way, the magnet achieved very high field quality of one unit of $10^{-4}$ at a radius of $25 \mathrm{~mm}$ with the center set off by up to $20 \mathrm{~mm}$.

The HERA IR magnets were operated with $4.5 \mathrm{~K}$ supercritical He. The performance in routine operation was excellent.

The technique of the very successful HERA low beta quadrupoles was further developed by BNL scientists and the technique has been successfully applied to the low beta quadrupoles of the Beijing Electron-Positron Collider BEPC-II [186], the KEK b-factory SuperKEKB [187] and designs for the interaction regions of the International Linear Collider [188] have bee produced and prototyped. The Fig. 10.15 shows a photograph taken during the winding of the low-beta quadruole for superKEKB in September 2013. This technology is now quite mature and is used for many of the advanced eRHIC interaction region magnets [177]. 


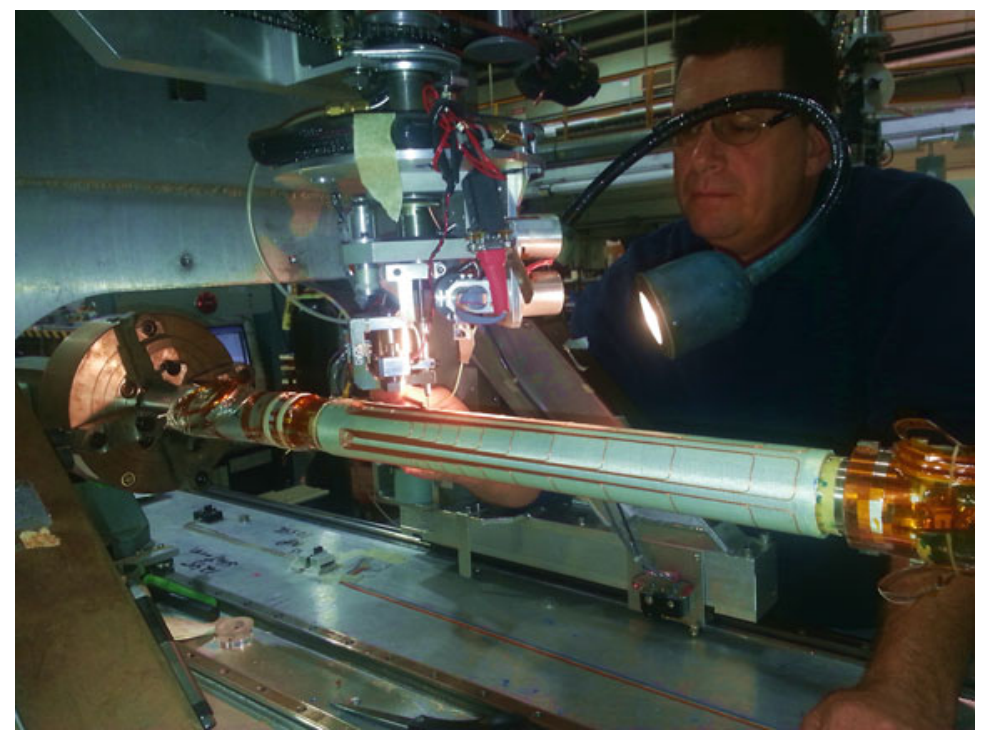

Fig. 10.15 Winding of the superKEKB coil in September 2013 (Courtesy Brett Parker, BNL)

\subsubsection{Conclusion}

The accelerator and technical solutions as well as the operational proceedures which enabled the successful operation of the HERA Lepton-Hadron collider are an important resource for the design of future colliders, in particular for the design of ring-based electron-ion colliders.

\subsection{LHC Layout and Performance to Date}

R. Bailey $\cdot$ J. Wenninger

\subsubsection{Introduction}

The Large Hadron Collider (LHC) [189] is a two-ring superconducting accelerator and collider installed in a $27 \mathrm{~km}$ underground tunnel at CERN, on the Swiss-French border near to Geneva. The tunnel was originally constructed for the Large Electron Positron collider (LEP), which operated from 1989 to 2000.

The LHC has two high luminosity experiments, ATLAS and CMS, designed for a peak luminosity of $10^{34} \mathrm{~cm}^{-2} \mathrm{~s}^{-1}$, one dedicated ion experiment, ALICE, and one 
Table 10.15 LHC basic beam parameters

\begin{tabular}{|c|c|c|c|}
\hline Parameter & Unit & Injection & Collision \\
\hline \multicolumn{4}{|l|}{ Beam data } \\
\hline Proton energy & $\mathrm{GeV}$ & 450 & 7000 \\
\hline Relativistic gamma $\gamma$ & & 479.6 & 7461 \\
\hline Number of particles per bunch & & \multicolumn{2}{|c|}{$1.15 \times 10^{11}$} \\
\hline Number of bunches & & \multicolumn{2}{|l|}{2808} \\
\hline Longitudinal emittance $(4 \sigma)$ & eVs & 1.0 & $2.5^{\mathrm{a}}$ \\
\hline Transverse normalized emittance & $\mu \mathrm{m} \mathrm{rad}$ & $3.5^{\mathrm{b}}$ & 3.75 \\
\hline Circulating beam current & A & \multicolumn{2}{|l|}{0.582} \\
\hline Stored energy per beam & MJ & 23.3 & 362 \\
\hline \multicolumn{4}{|l|}{ Peak luminosity related data } \\
\hline RMS bunch length ${ }^{\mathrm{c}}$ & $\mathrm{cm}$ & 11.24 & 7.55 \\
\hline RMS beam size at the IP1 and IP5 ${ }^{d}$ & $\mu \mathrm{m}$ & 375.2 & 16.7 \\
\hline RMS beam size at the IP2 and IP8 ${ }^{\mathrm{e}}$ & $\mu \mathrm{m}$ & 279.6 & 70.9 \\
\hline Geometric luminosity reduction factor $F^{\mathrm{f}}$ & & - & 0.836 \\
\hline Peak luminosity in IP1 and IP5 & $\mathrm{cm}^{-2} \mathrm{~s}^{-1}$ & - & $1.0 \times 10^{34}$ \\
\hline Peak luminosity per bunch in IP1 and IP5 & $\mathrm{cm}^{-2} \mathrm{~s}^{-1}$ & - & $3.5 \times 10^{30}$ \\
\hline
\end{tabular}

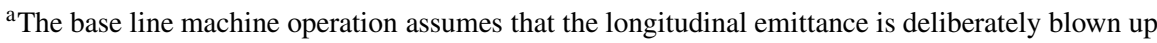
in the middle of the ramp in order to reduce the intra beam scattering growth rates

${ }^{\mathrm{b}}$ The emittance at injection energy refers to the emittance delivered to the LHC by the SPS without any increase due to injection errors and optics mis-match. The RMS beam sizes at injection assume the nominal emittance value quoted for top energy (including emittance blowup due to injection oscillations and mismatch)

${ }^{\mathrm{c}}$ Dimensions are given for Gaussian distributions. The real beam will not follow a Gaussian distribution but more realistic distributions do not allow analytic estimates for the IBS growth rates

${ }^{\mathrm{d}}$ The RMS beam sizes in IP1 and IP5 assume a $\beta$-function of $0.55 \mathrm{~m}$

${ }^{\mathrm{e}}$ The RMS beam sizes in IP2 and IP8 assume a $\beta$-function of $10 \mathrm{~m}$

${ }^{f}$ The geometric luminosity reduction factor depends on the total crossing angle at the IP. The quoted number assumes a total crossing angle of $285 \mu \mathrm{rad}$ in IR1 and IR5

experiment for B-physics, LHCb. The main parameters required to reach the high luminosity for ATLAS and CMS are given in Table 10.15.

The high beam intensities required for a luminosity of $L=10^{34} \mathrm{~cm}^{-2} \mathrm{~s}^{-1}$ exclude the use of anti-proton beams and a single common vacuum and magnet system for both circulating beams (as was done in the SPPbarS and the TEVATRON) and implies the use of two proton beams. To collide two beams of equally charged particles requires opposite magnet dipole fields in both beams. The LHC is therefore designed as a proton-proton collider with separate magnet fields and vacuum chambers in the main arcs and with common sections only at the insertion regions where the experimental detectors are located.

There is not enough room for two separate rings of magnets in the LEP tunnel. Therefore the LHC uses twin bore magnets which consist of two sets of coils and beam channels within the same mechanical structure and cryostat.

The peak beam energy in a storage ring depends on the integrated dipole field along the storage ring circumference. Aiming at peak beam energies of up to $7 \mathrm{TeV}$ 
inside the existing LEP tunnel implies a peak dipole field of $8.33 \mathrm{~T}$ and the use of superconducting magnet technology.

This presented quite a challenge; to make the most profitable use of the existing tunnel and to obtain the highest possible bending strength by exploiting the wellproven technology based on $\mathrm{Nb}$-Ti Rutherford cables. To meet this challenge and to find the cheapest solution compatible with the required performance needed a substantial R\&D program on magnets and associated technology. This was carried out between 1988 and 2001 by CERN in close collaboration with other European laboratories and European industry.

\subsubsection{Layout}

The basic layout of the LHC follows the LEP tunnel geometry and is depicted in Fig. 10.16. The LHC has eight arcs and straight sections, with dispersion suppressors in between.

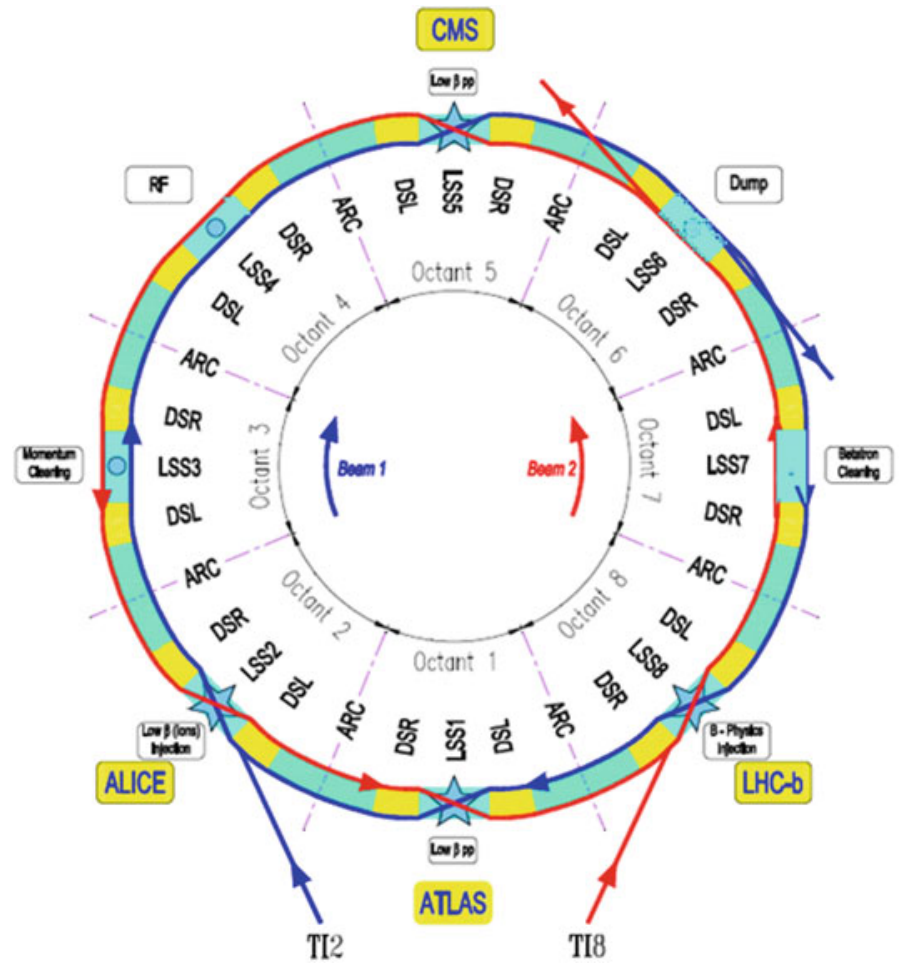

Fig. 10.16 Schematic layout of the LHC. Beam 1 (Blue) circulates clockwise. Beam 2 (Red) circulated counter-clockwise 


\subsubsection{The Straight Sections}

Each straight section is approximately $528 \mathrm{~m}$ long and can serve as an experimental or utility insertion. The two high luminosity experimental insertions are located at diametrically opposite straight sections: the ATLAS experiment is located at point 1 and the CMS experiment at point 5. Two more experimental insertions, for ALICE and $\mathrm{LHCb}$ are located at point 2 and point 8 respectively. These insertion regions also contain the injection systems, for Beam 1 arriving from the SPS through the transfer line TI2, and for Beam 2 arriving from the SPS through the transfer line TI8. The injection kick occurs in the vertical plane with the two beams arriving at the LHC from below the LHC reference plane. The beams only cross from one magnet bore to the other at these four locations. The remaining four straight sections do not have beam crossings. Insertion 3 and 7 each contain two collimation systems, for momentum cleaning and betatron cleaning respectively. Insertion 4 contains two RF systems: one independent system for each LHC beam. The straight section at point 6 contains the beam dump insertion where the two beams are vertically extracted from the machine using a combination of horizontally deflecting fast-pulsed ('kicker') magnets and vertically-deflecting double steel septum magnets. Each beam features an independent abort system.

The two beams share an approximately $130 \mathrm{~m}$ long common beam pipe along the interaction regions (IR). The exact length is $126 \mathrm{~m}$ in IR2 and IR8 which feature superconducting separation dipole magnets next to the triplet assemblies and $140 \mathrm{~m}$ in IR1 and IR5 which feature normal conducting and therefore longer separation dipole magnets next to the triplet assemblies. Together with the large number of bunches (2808 for each proton beam), and a nominal bunch spacing of $25 \mathrm{~ns}$, the long common beam pipe implies 34 parasitic collision points for each experimental insertion region (for four experimental IR's this implies a total of 136 unwanted collision points). Dedicated crossing angle orbit bumps separate the two LHC beams left and right from the central interaction point (IP) in order to avoid collisions at these parasitic collision points.

\subsubsection{The Arcs}

The arcs of the LHC are each made of 23 regular arc cells. The arc cells are $106.9 \mathrm{~m}$ long and are made out of two $53.45 \mathrm{~m}$ long half cells each of which contains one $5.355 \mathrm{~m}$ long cold mass ( $6.63 \mathrm{~m}$ long cryostat) short straight section (SSS) assembly and three $14.3 \mathrm{~m}$ long dipole magnets. The two apertures for Ring 1 and Ring 2 are separated by $194 \mathrm{~mm}$. The two coils in the dipole magnets are powered in series and all dipole magnets of one arc form one electrical circuit. The quadrupoles of each arc form two electrical circuits: all focusing quadrupole magnets in Ring 1 and Ring 2 are powered in series and all defocusing quadrupole magnets of Beam 1 and Beam 2 are powered in series. The optics of Beam 1 and Beam 2 in the arc cells is therefore strictly coupled via the powering of the main magnetic elements. 


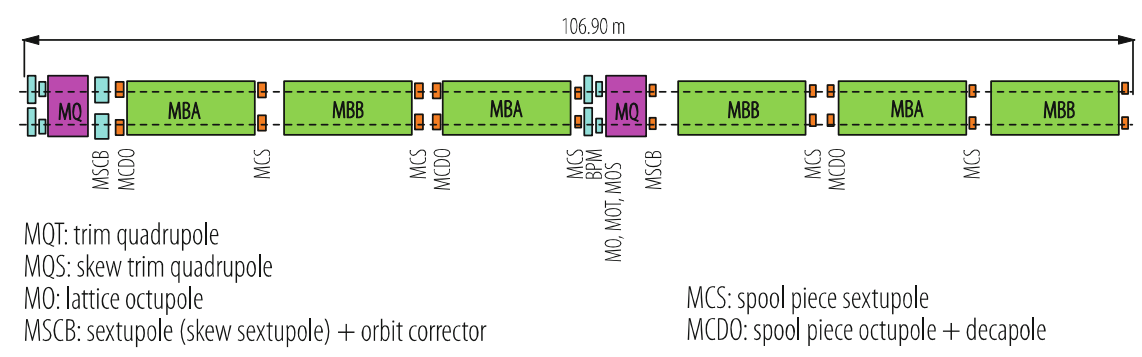

Fig. 10.17 The LHC arc cell layout

An LHC arc cell is also equipped with corrector magnets, which can be split into two distinct categories (see Fig. 10.17).

- The lattice corrector magnets attached on both sides of the main quadrupole magnets are installed in the Short Straight Section (SSS) cryostats.

- The spool-piece corrector magnets which are thin non-linear windings attached directly on the extremities of the main dipoles.

Contrary to the main dipole circuits and the two families (QF and QD) of lattice quadrupoles for which, in each sector, Ring 1 and Ring 2 are powered in series, the arc corrector magnets can be adjusted independently for the two beams.

\subsubsection{The Dispersion Suppressors}

A dispersion suppressor is located at the transition between an LHC arc and a straight section yielding a total of 16 dispersion suppressor sections. The aim of the dispersion suppressors is threefold:

- adapt the LHC reference orbit to the geometry of the LEP tunnel;

- cancel the horizontal dispersion arising in the arc and generated by the separation/recombination dipole magnets and the crossing angle bumps;

- help in matching the insertion optics to the periodic solution of the arc.

A generic design of a dispersion suppressor uses standard arc cells with missing dipole magnets. The LEP dispersion suppressor, which defines the geometry of the tunnel, was made of 3.5 cells with a $90^{\circ}$ phase advance, optimized to suppress the dispersion. With the 2.5 times longer LHC dipole and quadrupole magnets, only two LHC cells can be fitted in the dispersion suppressor tunnel.

\subsubsection{LSS1 and LSS5}

IR1 and IR5 house the high luminosity experiments of the LHC and are identical in terms of hardware and optics (except for the crossing-angle scheme: the crossing 


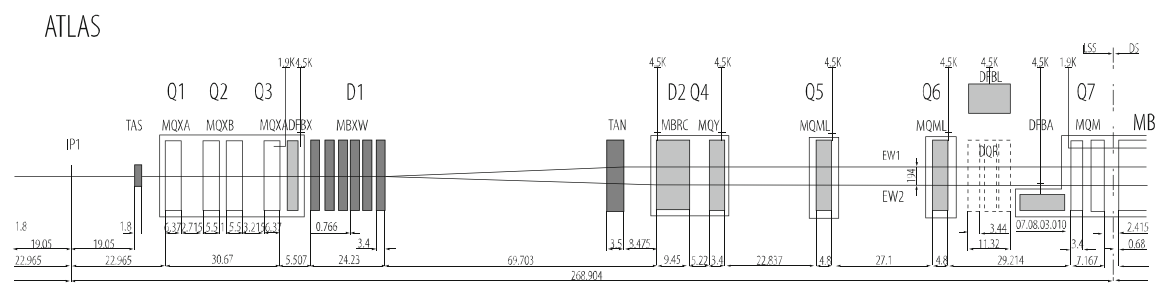

Fig. 10.18 Schematic layout of the right side of IR1

angle in IR1 is in the vertical plane and in IR5 in the horizontal plane). The small $\beta$-function values at the IP are generated with the help of a triplet quadrupole assembly. At the IP, the two rings share the same vacuum chamber, the same lowbeta triplet magnets and the D1 separation dipole magnets. The remaining matching section (MS) and the dispersion suppressor (DS) consist of double-bore magnets with separate beam pipes for each ring.

Figure 10.18 shows the schematic layout of IR1.

Apart from the DS the insertions are comprised of the following sections, given in order from the interaction point:

- A $31 \mathrm{~m}$ long superconducting low- $\beta$ triplet assembly operated at a temperature of $1.9 \mathrm{~K}$ and providing a nominal gradient of $205 \mathrm{~T} / \mathrm{m}$.

- A pair of separation/recombination dipoles separated by approximately $88 \mathrm{~m}$. The D1 dipole located next to the triplet magnets has a single bore and consists of six $3.4 \mathrm{~m}$ long conventional warm magnet modules yielding a nominal field of $1.38 \mathrm{~T}$. The following D2 dipole is a $9.45 \mathrm{~m}$ long, double bore, superconducting dipole magnet operating at a cryogenic temperature of $4.5 \mathrm{~K}$ with a nominal field of $3.8 \mathrm{~T}$. The bore separation in the D2 magnet is $188 \mathrm{~mm}$ and is thus slightly smaller than the arc bore separation.

- Four matching quadrupole magnets. The first quadrupole following the separation dipole magnets, $\mathrm{Q} 4$, is a wide-aperture magnet operating at a cryogenic temperature of $4.5 \mathrm{~K}$ and yielding a nominal gradient of $160 \mathrm{~T} / \mathrm{m}$. The remaining three quadrupole magnets are normal-aperture quadrupole magnets operating at a cryogenic temperature of $1.9 \mathrm{~K}$ with a nominal gradient of $200 \mathrm{~T} / \mathrm{m}$.

The triplet assembly features two different quadrupole designs: the outer two quadrupole magnets are made by KEK and require a peak current of $6450 \mathrm{~A}$ to reach the nominal gradient of $205 \mathrm{~T} / \mathrm{m}$, whereas the inner quadrupole block consists of two quadrupole magnets made by FNAL and requires a peak current of 10,630 A.

The triplet quadrupoles are powered by two nested power converters: one $8 \mathrm{kA}$ power converter powering all triplet quadrupole magnets in series and one $6 \mathrm{kA}$ power converter supplying additional current only to the central two FNAL magnets. The Q1 quadrupole next to the IP features an additional $600 \mathrm{~A}$ trim power converter. The triplet quadrupoles are followed by the separation/recombination dipoles, D1 and D2, which guide the beams from the IP into two separated vacuum chambers. 
Q4, Q5, Q6, Q7, Q8, Q9 and Q10 are individually powered magnets. The aperture of Q4 is larger to provide sufficient aperture for the crossing angle separation orbit. Two absorbers protect the cold magnets from particles leaving the IP. The TAS absorber protects the triplet quadrupole magnets and the TAN absorber, located in front of the D1 dipole magnet, protects the machine elements from neutral particles leaving the IP.

The matching section extends from Q4 to Q7 and the DS extends from Q8 to Q11. In addition to the DS, the first two trim quadrupoles of the first arc cell (QT12 and QT13) are also used for the matching procedure. All insertion and DS magnets are equipped with a beam screen [2]. The magnets left and right from the IP up to Q7 inclusive are placed symmetrically with respect to the IP. The positions of Q8, Q9 and Q10 left and right from the IP differ by approximately $0.5 \mathrm{~m}$ with respect to the IP due to the limited space in the DS.

\subsubsection{LSS2}

The straight section at IR2 houses the injection elements for Ring-1 as well as the ion beam experiment ALICE. During injection the optics must obey the special constraints imposed by the beam injection for Ring-1 and the geometrical acceptance in the interaction region (IR) must be large enough to accommodate both beams in the common part of the ring with a beam separation of at least $10 \sigma$.

Figures 10.19 and 10.20 show the schematic layout of IR2.

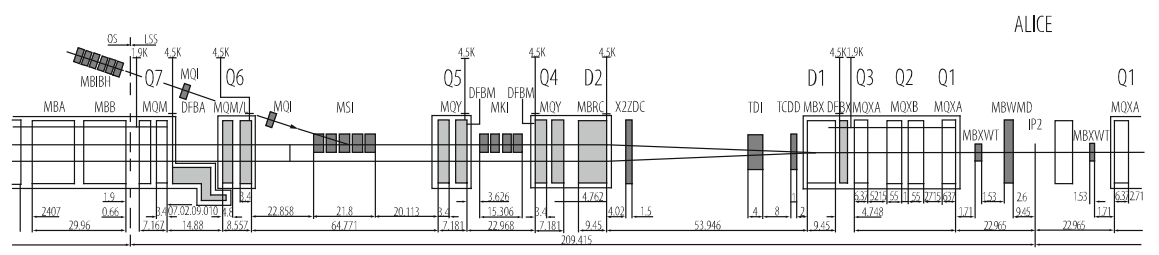

Fig. 10.19 Schematic layout of the left side of IR2

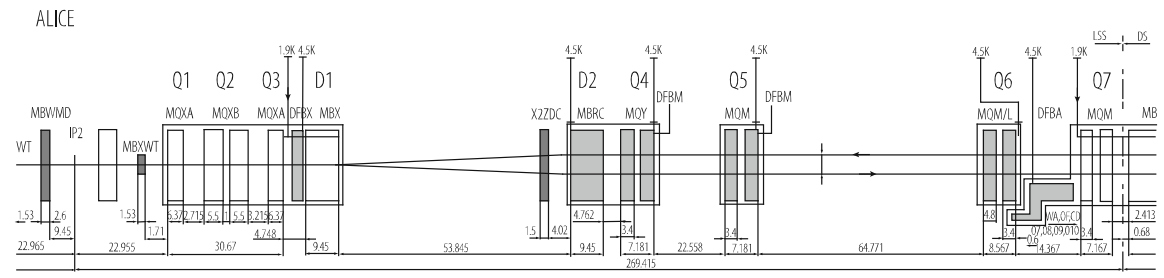

Fig. 10.20 Schematic layout of the right side of IR2 
Apart from the DS the insertions comprise the following sections, given in order from the interaction point:

- A $31 \mathrm{~m}$ long superconducting low- $\beta$ triplet assembly operated at $1.9 \mathrm{~K}$ and providing a nominal gradient of $215 \mathrm{~T} / \mathrm{m}$.

- A pair of $9.45 \mathrm{~m}$ long superconducting separation/recombination dipole magnets separated by approximately $66 \mathrm{~m}$.

- Four matching quadrupole magnets. The first two quadrupole magnets following the separation dipole magnets, Q4 and Q5, are wide aperture magnets operating at $4.5 \mathrm{~K}$ and yielding a nominal gradient of $160 \mathrm{~T} / \mathrm{m}$. The remaining two quadrupole magnets are normal aperture quadrupole magnets operating at $1.9 \mathrm{~K}$ with a nominal gradient of $200 \mathrm{~T} / \mathrm{m}$.

The triplet quadrupoles are powered in series and are followed by the separation/recombination dipoles D1 and D2, which guide the beams from the IP into two separated vacuum chambers. Q4, Q5, Q6, Q7, Q8, Q9 and Q10 are individually powered magnets. The aperture of $\mathrm{Q} 4$ is increased to provide sufficient aperture for the crossing-angle separation orbit. The aperture of Q5 left of the IP is increased to provide sufficient aperture for the injected beam. The injection septum MSI is located between Q6 and Q5 on the left-side of the IP and kicks the injected beam in the horizontal plane towards the closed orbit of the circulating beam (positive deflection angle). The injection kicker MKI is located between Q5 and Q4 on the left-hand side of the IP and kicks the injected beam in the vertical plane towards the closed orbit of the circulating beam (negative deflection angle). In order to protect the cold elements in case of an injection failure a large absorber (TDI) is placed $15 \mathrm{~m}$ upstream from the D1 separation/recombination dipole left from the IP. The TDI absorber is complemented by an additional shielding element $3 \mathrm{~m}$ upstream of the D1 magnet and two additional collimators installed next to the Q6 quadrupole magnet. In order to obtain an optimum protection level in case of injection errors the vertical phase advance between MKI and TDI must be $90^{\circ}$ and the vertical phase advance between the TDI and the two auxiliary collimators must be an integer multiple of $180^{\circ} \pm 20^{\circ}$.

The matching section extends from Q4 to Q7 and the DS extends from Q8 to Q11. In addition to the DS, the first two trim quadrupoles of the first arc cell (QT12 and QT13) are also used for the matching procedure. All magnets of the DS are equipped with a beam screen. The magnets left and right from the IP up to Q7 inclusive are placed symmetrically with respect to the IP. The positions of Q8, Q9 and Q10 left and right from the IP differ by approximately $0.5 \mathrm{~m}$ with respect to the IP due to the limited space in the DS.

\subsubsection{LSS8}

IR8 houses the $\mathrm{LHCb}$ experiment and the injection elements for Beam 2. The small $\beta$-function values at the IP are generated with the help of a triplet quadrupole assembly. At the IP, the two rings share the same vacuum chamber, the same low- 


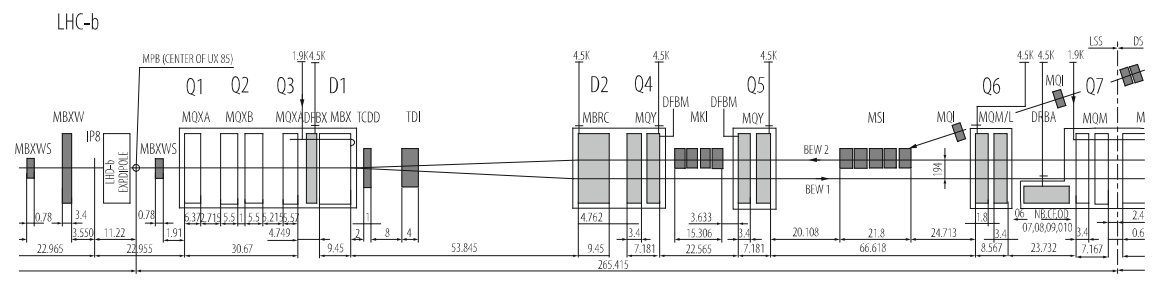

Fig. 10.21 Schematic layout of the right side of IR8

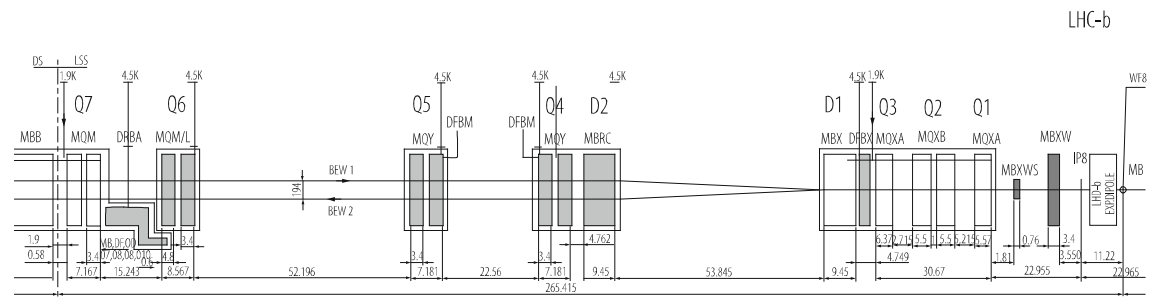

Fig. 10.22 Schematic layout of the left side of IR8

beta triplet magnets and the D1 separation dipole magnet. The remaining matching section (MS) and the DS consist of double-bore magnets with separate beam pipes for each ring.

Figures 10.21 and 10.22 show the schematic layout of IR8.

Apart from the DS the insertions contain the following sections, given in order from the interaction point:

Three warm dipole magnets compensate the deflection generated by the $\mathrm{LHCb}$ spectrometer magnet.

- A $31 \mathrm{~m}$ long superconducting low- $\beta$ triplet assembly operated at $1.9 \mathrm{~K}$ and providing a nominal gradient of $205 \mathrm{~T} / \mathrm{m}$.

- A pair of separation/recombination dipole magnets separated by approximately $54 \mathrm{~m}$. The D1 dipole located next to the triplet magnets is a $9.45 \mathrm{~m}$ long singlebore superconducting magnet. The following D2 dipole is a $9.45 \mathrm{~m}$ long, double bore, superconducting dipole magnet. Both magnets are operated at $4.5 \mathrm{~K}$. The bore separation in the D2 magnet is $188 \mathrm{~mm}$ and is thus slightly smaller than the arc bore separation.

- Four matching quadrupole magnets. The first quadrupole following the separation dipole magnets, $\mathrm{Q} 4$, is a wide aperture magnet operating at $4.5 \mathrm{~K}$ and yielding a nominal gradient of $160 \mathrm{~T} / \mathrm{m}$. The remaining three matching section quadrupole magnets are normal aperture quadrupole magnets operating at $1.9 \mathrm{~K}$ with a nominal gradient of $200 \mathrm{~T} / \mathrm{m}$.

- The injection elements for Beam 2 on the right hand side of IP8. The $21.8 \mathrm{~m}$ long injection septum consists of five modules and is located between the Q6 and Q5 quadrupole magnets on the right-hand side of the IP. The $15 \mathrm{~m}$ long 
injection kicker consists of four modules and is located between the Q5 and Q4 quadrupole magnets on the right-hand side of the IP. In order to protect the cold elements in case of injection failure a large absorber (TDI) is placed $15 \mathrm{~m}$ in front of the D1 separation/recombination dipole magnet right from the IP. The TDI is complemented by an additional shielding element between the TDI and D1 magnet (placed $3 \mathrm{~m}$ in front of D1) (TCDD) and by two additional collimators placed on the transition of the matching section left from the IP to the next DS section.

In order to provide sufficient space for the spectrometer magnet of the $\mathrm{LHCb}$ experiment, IP8 is shifted by 15 half RF wavelengths ( 3.5 times the nominal bunch spacing $\sim 11.25 \mathrm{~m}$ ) towards IR7. This shift of the IP has to be recuperated before the beam returns to the dispersion suppressor sections and implies a non-symmetric magnet layout in the matching section.

\subsubsection{LSS3 and LSS7}

The insertion IR3 houses the momentum cleaning systems of both beams, while IR7 houses the betatron cleaning systems of both beams. Particles with a large momentum offset are scattered by the primary jaw of IR3. Particles with a large H, V or combined $\mathrm{H}-\mathrm{V}$ betatron amplitudes are scattered by the primary collimator jaws in IR7. In both cases the scattered particles are absorbed by secondary collimators.

Figure 10.23 shows the schematic layout of IR7.

The dispersion suppressor extends from Q8 to Q11. In addition to the DS, the first two trim quadrupoles of the first arc cell (QT12 and QT13) are also used for the matching procedure. All cryo-magnets are equipped with a beam screen. In IR3 and IR7, the underground galleries are not wide enough to house many high current power supplies. Therefore, contrary to the layout of the other IR's, the DS quadrupoles (Q7, Q8, Q9 and Q10) are made of a MQ+MQTL assembly (MQ + 2 MQTL at Q9) where the MQ's magnets are powered in series with the main arc quadrupoles. To avoid producing two kinds of MQ+MQTL assemblies, the dispersion suppressors left and right from the IP are not mirror symmetric with respect to each other. Instead, the DS quadrupole assemblies have the same orientation in the dispersion suppressors left and right from the IP and the MQ positions differ by approximately $0.5 \mathrm{~m}$ with respect to the IP in the two DS.

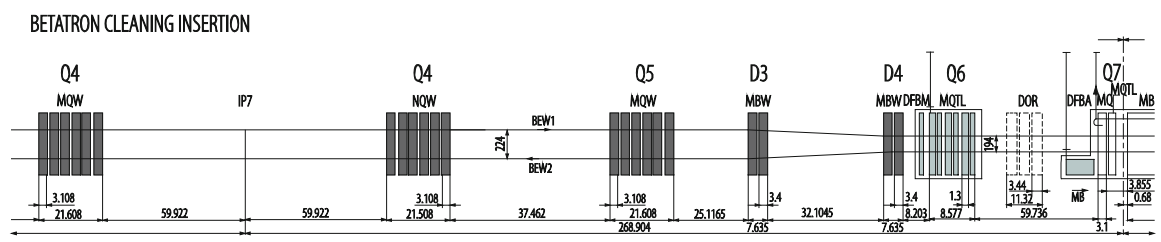

Fig. 10.23 Schematic layout of the right side of IR7 
The layout of the Long Straight Section between Q7L and Q7R is mirror symmetric with respect to the IP. The right side of IR7 is shown in Fig. 10.23. This allows the symmetrical installation for the collimators of the two beams and minimizes the space conflicts in the insertion. Starting from Q7 left, the quadrupole Q6 (made of six superconducting MQTL modules) is followed by a dog-leg structure made of two sets of MBW warm single bore wide aperture dipole magnets (two warm modules each). The dogleg dipole magnets are labeled D3 and D4 in the LHC sequence with D3 being the dipole closer to the IP. The Primary Collimators are located between the D4 and D3 magnets, allowing neutral particles produced in the jaws to point out of the beam line, and most charged particles to be swept away. The inter-beam distance between the dogleg assemblies left and right from the IP is $224 \mathrm{~mm}$, i.e. $30 \mathrm{~mm}$ larger than in the arc. This increased beam separation allows a substantially higher gradient in the Q4 and Q5 quadrupoles which are made out of six warm MQW modules. The space between Q5 left and right from the IP is used to house the secondary collimators at adequate phase advances with respect to the primary collimators.

The Q4 and Q5 quadrupoles left and right from the IP are powered in series. The warm dual-bore MQW quadrupole cannot be powered with different currents for each magnet aperture because the field quality is degraded to an unacceptable level even for a small imbalance in the field of the two apertures. The current must be equal or of opposite value in the bores to provide a good field quality. In order to obtain the required flexibility for the optics, two different kinds of powering schemes are used for the Q4 and Q5 quadrupole units. The magnets are identical, but in the MQWA type magnet the field is identical in both apertures, while in the MQWB type magnet, the field is opposite for both apertures. Each Q4 and Q5 assembly is made of five MQWA and one MQWB module. The nominal gradient of the MQWB unit is limited to $29.6 \mathrm{~T} / \mathrm{m}$ while it can reach $35 \mathrm{~T} / \mathrm{m}$ in the MQWA unit. This powering scheme breaks the exact antisymmetry by $29 \%$ providing enough flexibility to satisfy all the optics constraints. Again, Q5AL+Q5AR and $\mathrm{Q} 5 \mathrm{BL}+\mathrm{Q} 5 \mathrm{BR}$ respectively are powered in series. As a by-product, this freedom in the straight section allows the trim strength needed in the DS to be limited so that regular MQTL's can be used.

In IR3, the most difficult constraint was to generate a large dispersion function in the straight section. Since the layout of the DS cannot be changed in IR3 this constraint means that the natural dispersion suppression generated in the DS is over compensated. To this end Q6 and Q5 were moved towards each other by a substantial amount, thus shrinking the space granted to the dog-leg structure D4D3. It was therefore necessary to add a third MBW element to D3 and D4 in IR3. Apart from this IR3 and IR7 are identical.

\subsubsection{LSS4}

IR4 houses the RF and feed-back systems as well as some of the LHC beam instrumentation. 


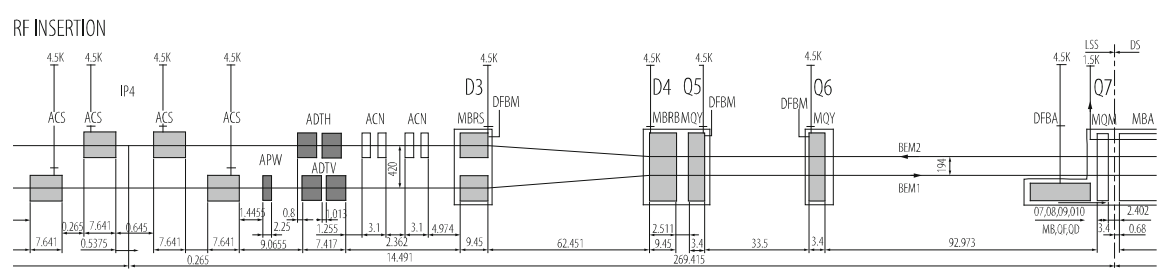

Fig. 10.24 Schematic layout of the right side of IR4

Figure 10.24 shows the schematic layout of IR4.

There are six superconducting quadrupole magnets in the straight section: Q5 Q6 Q7 on the left- and Q5 Q6 Q7 on the right-hand side of the IP. The outer dogleg dipoles, D4, sit next to the Q5 quadrupole magnets on each side of the IP. The RF cavities sit between the inner dogleg dipoles, D3. The layout of the DS, between Q7 and Q11, is identical to that in IR1 and IR5.

\subsubsection{LSS6}

IR6 houses the beam abort systems for Beam 1 and Beam 2. The beam extraction from the LHC is done by kicking the circulating beam horizontally into an iron septum magnet which deflects the beam in the vertical direction away from the machine components to absorbers in a separate tunnel. Each ring has its own system and both are installed in IR6.

Figure 10.25 shows the schematic layout of IR6.

In each of the dispersion suppressors up to six quadrupoles can be used for matching. The total of 16 quadrupoles is more than necessary to match both $\beta$ functions, the dispersion (both at the crossing point and in the arc) and adjust the phases. Although this number of parameters seems considerable, their variation is strongly limited by the aperture constraints which set limits on the $\beta$-functions and the dispersion inside the insertion. Special detection devices protect the extraction septum and the LHC machine against losses during the extraction process. The TCDS absorber is located in front of the extraction septum and the TCDQ in front of the Q4 quadrupole magnet downstream of the septum magnet.

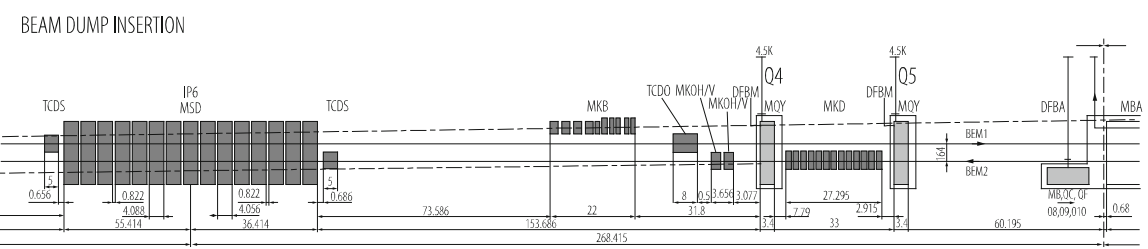

Fig. 10.25 Schematic layout of the right side of IR6 


\subsubsection{Performance}

The LHC was first operated with beam for short periods in 2008 and 2009. In 2010 a first experience with the machine was gained at a beam energy of $3.5 \mathrm{TeV}$, and moderate beam intensity of up to around 200 bunches of $1.1 \times 10^{11}$ protons per bunch (ppb). The reduced energy was chosen such as to minimize risks associated to the quality issue of the soldering of the busbar cables in the magnet interconnections. In 2011 the beam intensity was pushed to around 1400 bunches of $1.4 \times 10^{11} \mathrm{ppb}$ while 2012 was dedicated to luminosity production with higher bunch intensities $1.6 \times 10^{11} \mathrm{ppb}$ and a beam energy of $4 \mathrm{TeV}$. A bunch spacing of $50 \mathrm{~ns}$ was used in 2011 and 2012 to minimize the complication of electron clouds. The first operation period is commonly referred to as run 1 . In early 2013 beam operation was stopped for a 2-year long shutdown (LS1) to consolidate the magnet interconnections in view of reaching the design beam energy. Many details on LHC operation during Run 1 may be found in [190].

Beam operation resumed in 2015 at $6.5 \mathrm{TeV}$ following a dipole training campaign of 169 quenches at the end of Long Shutdown 1 (LS1). The LHC experiments expressed a strong preference for beams with $25 \mathrm{~ns}$ bunch spacing, as opposed to the $50 \mathrm{~ns}$ spacing used in 2011-2012, as this would result in a too high number of inelastic collisions per crossing (pile-up). On the machine side $25 \mathrm{~ns}$ beams pose additional challenges, e.g. the formation of electron clouds (eclouds) in the vacuum chamber and a higher number of fast loss events, named Unidentified Falling Objects (UFOs). Given the number of new territories had to be explored, 2015 was considered a re-commissioning and a learning year, dedicated to preparing the machine for full luminosity production in 2016-2018. In 2016 the machine performance was pushed for the first time above the design luminosity of $10^{34} \mathrm{~cm}^{-2} \mathrm{~s}^{-1}$, and by the end of that year the design had been exceeded by $40 \%$. This excellent performance was possible thanks to beams of much lower emittance produced by the LHC injector chain, coupled to a reduced $\beta^{*}$ of $40 \mathrm{~cm}$ as compared to the design value of $55 \mathrm{~cm}$. In 2017 the performance could be pushed further with more than twice the design luminosity with a further reduction of the beam emittances and of $\beta^{*}$ to $30 \mathrm{~cm}$. The performance in 2017 was so exceptional that the luminosities of ATLAS and CMS had to levelled down to limit the event pile-up to 60. Figures 10.26 and 10.27 present the evolution of the peak and of the integrated luminosity between 2011 and 2017. A summary of the main parameters is presented in Table 10.16. Details on operation in LHC Run 2 up to and including 2016 can be found in [191].

The remarkable performance of LHC during run 2 between 2015 and 2017 was achieved despite unexpected limitations. In 2016 the SPS beam dump was damaged when high intensity LHC beams were dumped onto the block at $450 \mathrm{GeV}$, developing a vacuum leak. Because it was not practical to exchange the dump during 


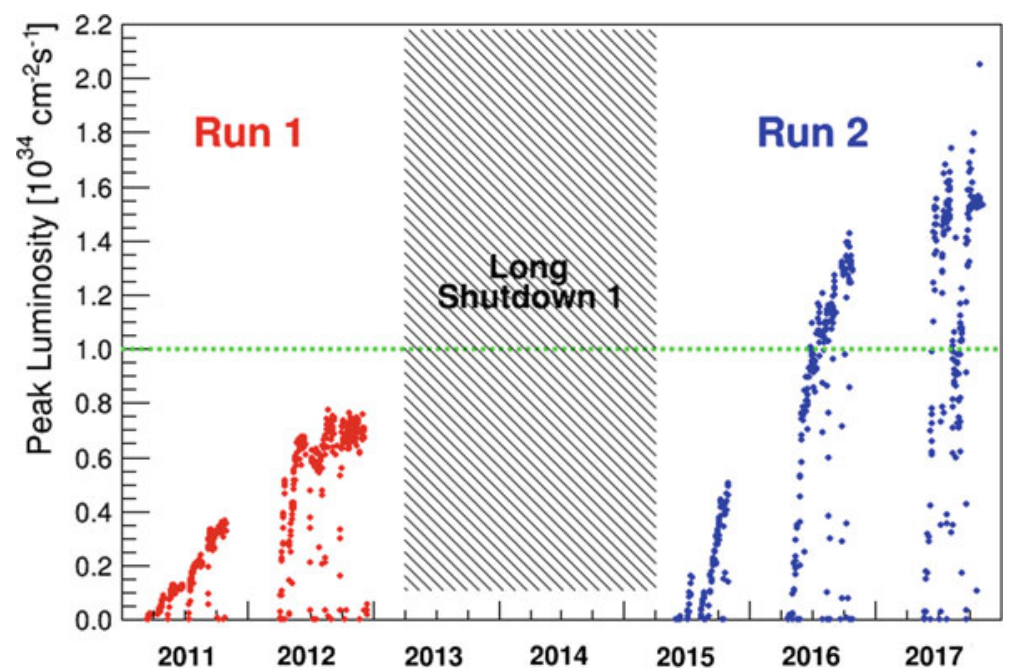

Fig. 10.26 Peak luminosity performance of the LHC in ATLAS and CMS between 2011 and 2017. In July 2016 the LHC reached the design luminosity of $1 \times 10^{34} \mathrm{~cm}^{-2} \mathrm{~s}^{-1}$

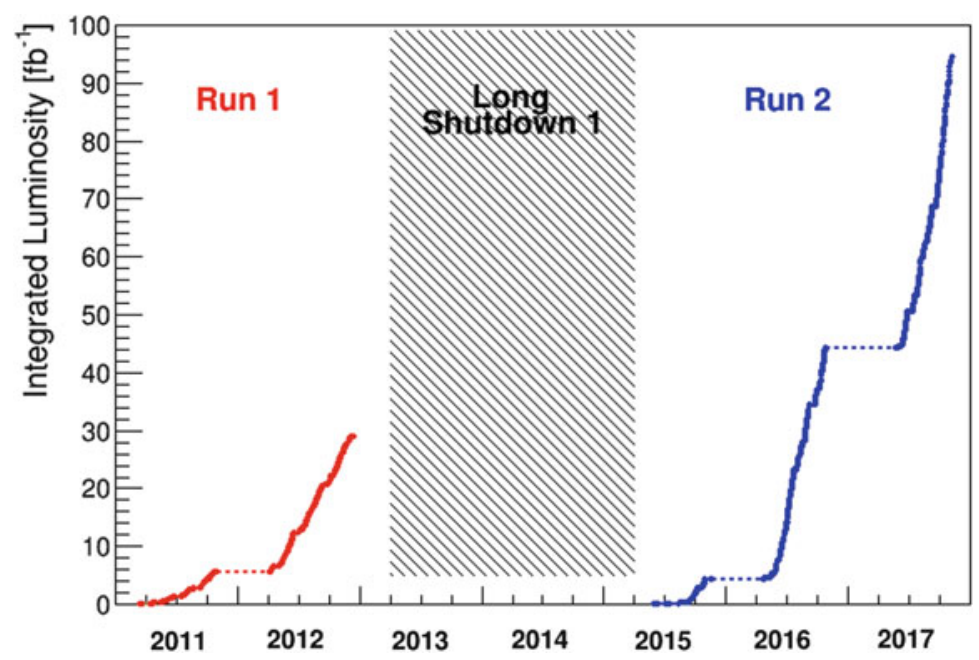

Fig. 10.27 Integrated luminosity delivered to the ATLAS and CMS experiments between 2011 and 2017

the run, the LHC beams were limited to 144 bunches during that year. This limitation was lifted in 2017 when a new dump was installed in the SPS. Unfortunately, an undetected vacuum pumping problem during the cool down of one LHC sector brought another limitation for the 2017 run. A few liters of Air introduced by a 
Table 10.16 Performance reach in 2010 and 2011

\begin{tabular}{l|l|l|l|l|l}
\hline Parameter & Unit & 2012 & 2016 & 2017 & Design \\
\hline Energy & $\mathrm{TeV}$ & 4 & 6.5 & 6.5 & 7 \\
\hline Bunch intensity & $10^{10}$ & 16 & 11 & 12.5 & 11.5 \\
\hline Bunches per beam & & 1380 & 2556 & 2556 & 2802 \\
\hline Emittance & $\mu \mathrm{m}$ & 2.40 & 2.3 & 2.0 & 3.75 \\
\hline$\beta_{*}$ & $\mathrm{~m}$ & 0.6 & 0.4 & 0.3 & 0.55 \\
\hline Luminosity 1 and 5 & $\mathrm{cm}^{-2} \mathrm{~s}^{-1}$ & $7 \times 10^{33}$ & $1.5 \times 10^{34}$ & $2 \times 10^{34}$ & $1 \times 10^{34}$ \\
\hline
\end{tabular}

vacuum pump issue condensed as ice on the vacuum beam chamber. In the presence of very high intensity beams local losses would develop leading to beam dumps. Fortunately, it was possible to restore operation of the LHC with a low electron cloud variant of the $25 \mathrm{~ns}$ spacing LHC beam [192].

\section{References}

1. E. Regenstreif: Report CERN 62-03 (1962).

2. A. Hermann: History of CERN, Vol. I, North-Holland (1987).

3. U. Mersits, in: A. Hermann, et al.: History of CERN, Vol. II, North-Holland (1990)

4. S. Gilardone, D. Manglunki (eds.): Fifty Years of the CERN Proton Synchrotron, CERN2011-004.

5. D. Simon, J.P. Riunaud: Proc. 17th Intern. Conf. High-Energy Accelerators, Accelerator Catalogue, I. Meshkov (ed.), Dubna (1998), p. 72

6. J. Coupard et al. (eds): LIU TDR Vol 1, CERN-ACC-2014-0337 15 December 2014

7. F. Gerigk, M. Vretenar (eds): LINAC4 TDR, CERN-AB-2006-084 ABP/RF

8. O. Brüning et al. (eds): LHC Design Report Vol. 1, CERN-2004-003

9. M. Giovanni (ed.): Multi Turn Eextraction design report, CERN-2006-011

10. J. Bernhard et al.: Proc. IPAC'18, IPAC2018, Vancouver, Canada doi:10.18429/JACoWIPAC2018-TUPAF023

11. D. Simon: Proc. EPAC, Sitges (1996), p. 295.

12. N.C. Christofilos: unpublished manuscript (1950).

13. E.D. Courant, M.S. Livingston, H.S. Snyder: Phys. Rev. 88 (1952) 1190.

14. G.K. Green: Proc. Intern. Conf. High Energy Accelerators, M.H. Blewett (ed.), Brookhaven (1961), p. 39.

15. M. Plotkin: Brookhaven Nat. Lab. (1991) report-45058.

16. D.S. Barton: IEEE Trans. Nucl. Sci NS-30(4) (1983)2787.

17. L. Ahrens: HEACC'92, Intern. J. Mod. Phys. A (Proc. Suppl.) (1993) 109.

18. J.M. Brennan: IEEE Proc. Part. Accel. Conf., New York (1999), p. 614.

19. D.I. Lowenstein: Proc. 17th Intern. Conf. High-Energy Accelerators, Accelerator Catalogue, I. Meshkov (ed.), Dubna (1998), p. 72.

20. J.M. Brennan: IEEE Part. Accel. Conf., Dallas (1995), p. 1489.

21. K.A. Brown: Proc. IEEE Part. Accel. Conf., Portland (2003), p. 1545.

22. A. Zelenski: Proc. EPAC08, Genoa (2008), p. 1010.

23. Ya.S. Derbenev, A.M. Kondratenko: Sov. Phys. Dokl. 20 (1976) 562. Ya.S. Derbenev, et al.: Part. Accel. 8 (1978) 115.

24. T. Roser: 8th Intern. Symp. High-Energy Spin Physics, Particle and Fields Series 37, Minneapolis, MN (1988), p. 1442. 
25. L.A. Huang: Proc. IEEE Part. Accel. Conf., Vancouver (2009), p. 4251.

26. V. Schoefer: Proc. IEEE Part. Accel. Conf., New York (2011), to be published.

27. C.J. Gardner: Proc. IEEE Part. Accel. Conf., Albuquerque (2007), p. 1862.

28. A. Pikin, et al.: Proc. Intern. Symp. Electron Beam Ion Sources and Traps, IOP Publishing, Stockholm (2010). http://iopscience.iop.org/1748-0221/5/09/C09003/pdf/ jinst10_09_c09003.pdf

29. J. Alessi: Proc. IEEE Part. Accel. Conf., New York (2011), to be published.

30. V.V. Vladimirski, et al.: Proc. 4th Intern. Conf. High-Energy Accelerators, Dubna (1963), p. 233.

31. Institute of High Energy Physics, et al.: Proc. 6th Intern. Conf. High-Energy Accelerators, Cambridge (1967), p. 248.

32. E.F. Troyanov: Proc. 17th Intern. Conf. High-Energy Accelerators, Accelerator Catalogue, I. Meshkov (ed.), Dubna (1998), p. 29.

33. S. Ivanov: Proc. Russian Particle Accelerator Conf., Zvenigorod (2008), p. 130.

34. V.A. Teplyakov: Proc. 17th Intern. Conf. High-Energy Accelerators, Accelerator Catalogue, I. Meshkov (ed.), Dubna (1998), p. 31.

35. A.S. Gurevich: Proc. 17th Intern. Conf. High-Energy Accelerators, Accelerator Catalogue, I. Meshkov (ed.), Dubna (1998), p.30.

36. S. Ivanov. Proc. Russian Particle Accelerator Conf., Protvino (2010), p. 27.

37. A.G. Afonin: Proc. Russian Particle Accelerator Conf., Protvino (2010), session WECHX01.

38. S. Ivanov et al. Advances of Light-Ion Acceleration Program in the U70. Proc. 23rd Russian Particle Accelerators Conference RUPAC-2012, St.-Petersburg, 2012, pp. 100-102.

39. S. Ivanov, O. Lebedev. Transverse Noise Blow-up of the Beam in the U-70 Synchrotron. Instruments and Experimental Technique, Vol. 56, No. 3, 2013, pp. 249-255.

40. S. Ivanov, O. Lebedev. Attaining Square-Wave Stochastic Slow Extraction Spills from the U-70 Synchrotron. Instruments and Experimental Techniques, 2015, Vol. 58, No. 4, pp. 456464.

41. D.W. Kerst: Symp. High-Energy Accel. and Pion Physics, CERN (1956), CERN Report 5626, p. 36.

42. G.K. O'Neill: Symp. High-Energy Accel. and Pion Physics, CERN (1956), CERN Report 56-26, p. 64.

43. K.R. Symon, A.M. Sessler: Symp. High-Energy Accel. and Pion Physics, CERN (1956) CERN Report 56-26, p. 44.

44. CERN Study Group New Accelerators: Report CERN/542 (1964).

45. A. Hofmann: Proc. 11th Intern. Conf. High-Energy Accelerators, Accelerator Catalogue, J.H.B. Madsen, P.H. Standley (eds.), CERN (1980), p. 44.

46. K. Johnsen: CERN Report 84-13 (1984).

47. K. Johnsen: Proc. 5th Intern. Conf. High-Energy Accelerators, Frascati (1965), p. 3.

48. G. Plass: CERN Internal Report NPA/Int.61-8 (1961).

49. J.B. Adams: Proc. 8th Intern. Conf. High-Energy Accelerators, CERN (1971), p. 25.

50. J.B. Adams: Proc. 10th Intern. Conf. High-Energy Accelerators, Protvino (1977), p. 17.

51. K.-H. Kissler: Proc. $17^{\text {th }}$ Intern. Conf. High-Energy Accelerators, Accelerator Catalogue, I. Meshkov (ed.), Dubna (1998), p. 8.

52. H. Haseroth: Phys. Reports 403-404 (2004) 27.

53. LEP Injector Study Group: Report CERN-LEP/TH/83-29 (1983).

54. M. Benedikt, et al. (eds.): LHC Design Report, Vol. III, CERN-2004-003.

55. C. Rubbia, et al.: Proc. Intern. Neutrino Conf., Aachen (1976), p. 683.

56. S. van der Meer: CERN Internal Report ISR-PO/72-31 (1972).

57. P. Bramham, et al.: Nucl. Instrum. Meth. 125 (1975) 201.

58. G. Carron, et al.: Proc. Part. Accel. Conf., San Francisco (1979), p. 3456.

59. H. Koziol, D. Möhl: Phys. Reports 403-404 (2004) 91.

60. G. Brianti: Proc. 14th Conf. High-Energy Accelerators, Accelerator Catalogue, S. Kurokawa (ed.), Tsukuba (1989), p. 56.

61. J. Gareyte: Proc. 11th Intern. Conf. High-Energy Accel., CERN (1980), p. 79. 
62. H. Edwards: Annu. Rev. Nucl. Part. Sci. 35 (1985) 605.

63. M. Church: Proc. 17th Intern. Conf. High-Energy Accelerators, Accelerator Catalogue, I. Meshkov (ed.), Dubna (1998), p. 62.

64. F.R. Huson: Proc. 10th Intern. Conf. High-Energy Accelerators, Protvino (1977), p. 30.

65. S. Holmes (ed.): Report FNAL-TM-2484 (1998).

66. M. Church: Proc. Eur. Accelerator Conf., Paris (2002), p. 11.

67. A. Valishev, et al.: Proc. 23rd Particle Accelerator Conf., Vancouver (2009), p. 4230.

68. S.D. Holmes and V.D. Shiltsev, Annual Review of. Nuclear. and Particle Science, Vol.63, p. 435 .

69. M. Harrison, S. Peggs, T. Roser: Annu. Rev. Nucl. Part. Sci. 52 (2002) 425.

70. M. Harrison, et al.: Nucl. Instrum. Meth. A 499 (2003) 235.

71. M. Blaskiewicz, J.M. Brennan, K. Mernick: Phys. Rev. Lett. 105 (2010) 094801.

72. W. Fischer, et al.: Phys. Rev. ST Accel. Beams 11 (2008) 041002.

73. C. Montag, et al.: Phys. Rev. ST Accel. Beams 5 (2002) 084401.

74. W. Fischer, et al.: Proc. EPAC08, Genoa, Italy (2008), p. 1616.

75. A. Zelenski, J. Alessi, A. Kponou, D. Raparia: Proc. EPAC08, Genoa, Italy (2008) p. 1010.

76. H. Huang, et al.: Phys. Rev. ST Accel. Beams 7 (2004) 071001.

77. F. Lin, et al.: Phys. Rev. ST Accel. Beams 10 (2007) 044001.

78. Ya.S. Derbenev, A.M. Kondratenko: Part. Accel. 8 (1978) 115.

79. S.Y. Lee: Spin dynamcs and Snakes in Synchrotrons, World Scientific, Singapore (1997).

80. A. Bazilevsky, et al.: Proc. EPAC08, Genoa, Italy (2008), p. 1140.

81. M. Bai, et al.: Phys. Rev. Lett. 96 (2006) 174801.

82. W. Fischer, et al.: Phys. Rev. Lett. 115 (2015) 264801.

83. A. Fedotov et al.: Proc. NAPAC2016, Chicago, IL, USA (2016), p. 867

84. H. Blosser: Handbook of Accelerator Physics and Engineering, World Scientific (2006) 13.

85. E. Wilson: Handbook of Accelerator Physics and Engineering, World Scientific (2006) 57.

86. M. Tigner: Nuovo Cimento 37 (1965) 1228.

87. J. Seeman: Nonlinear Dynamics Aspects of Particle Accelerators, Springer-Verlag, Proc. 247 (1985) 121.

88. J. Rees: Handbook of Accelerator Physics and Engineering, World Scientific (2006) 11.

89. C. Bernardini, et al: High Energy Accelerator Conf. (1961) 256.

90. G. Budker, et al: J. Nucl. Energy C 8 (1966) 676.

91. G. O’Neill: High Energy Accelerator Conf. (1961) 247.

92. V. Auslender, et al: High Energy Accelerator Conf. (1965).

93. A. Skrinsky: Particle Accelerator Conf. (1995) 14.

94. G. Arzelier, et al: VIII High Energy Accelerator Conf. (1971) 127.

95. ADONE Group: Particle Accelerator Conf. (1971) 217.

96. J. Paterson, et al: Particle Accelerator Conf. (1971) 196.

97. J. Paterson, et al: Particle Accelerator Conf. (1975) 1366.

98. G. Tumaikin, et al: X High Energy Accelerator Conf. (1977) 443.

99. H. Nesemann, et al: Particle Accelerator Conf. (1983) 1998.

100. J. LeDuff, et al: XI High Energy Accelerator Conf. (1980) 566.

101. G. Voss, et al: XI High Energy Accelerator Conf. (1980) 748.

102. B. McDaniel, et al: Particle Accelerator Conf. (1981) 1984.

103. D. Rubin, et al: Particle Accelerator Conf. (1995) 481.

104. A. Blinoz, et al: XII High Energy Accelerator Conf. (1983) 183.

105. R. Helm, et al: Particle Accelerator Conf. (1983) 2001.

106. T. Nishikawa: XII High Energy Accelerator Conf. (1983) 143.

107. N. Phinney, et al: Particle Accelerator Conf. (1999) 3384.

108. J. Xu, et al: XII High Energy Accelerator Conf. (1983) 157.

109. S. Myers: Intern. Particle Accelerator Conf. (2010) 3663.

110. C. Milardi, et al: Particle Accelerator Conf. (2009) 80.

111. P. Raimondi: $2^{\text {nd }}$ SuperB Meeting, Frascati (2006).

112. J. Seeman, et al: Eur. Particle Accelerator Conf. (2008) 946. 
113. M. Tanaka, et al: Intern. Particle Accelerator Conf. (2011) 3735.

114. Q. Qin, et al: Intern. Particle Accelerator Conf. (2011) 3708.

115. Chinese Academy of Sciences: https://phys.org/news/2016-04-bepcii-luminosity-world$11033 \mathrm{~cm} 2 \mathrm{~s} . \mathrm{html}$.

116. D. Shwartz, et al: Russ. Particle Accelerator Conf. (2010) 1.

117. Y. Rogovsky, et al: Physcis and Technique of Accelerators, Springer, 2014, Vol. 11, No. 5, pp. 651-655.

118. H. Koiso: Intern. Particle Accelerator Conf. (2017) 1275.

119. P. Oddone: Proc. UCLA Workshop Linear Collider BB Factory Conceptual Design, D. Stork (ed.), (1987), p. 243.

120. K. Hirata, E. Keil: Nucl. Instrum. Meth. A 292 (1990) 156.

121. PEP-II: An Asymmetric B Factory. Conceptual Design Report, SLAC-418, QCD183:S56:1993 (1993).

122. KEKB B-Factory Design Report, KEK Report 95-7 (1995).

123. J. Seeman: Conf. Proc. C 0806233 (2008) TUXG01.

124. Y. Funakoshi, T. Abe, K. Akai, Y. Cai, K. Ebihara, K. Egawa, A. Enomoto, J. Flanagan, et al.: Conf. Proc. C 100523 (2010) WEOAMH02.

125. A. Piwinski: IEEE Trans. Nucl. Sci. 24 (1977) 1408.

126. R.B. Palmer: Proc. 1988 DPF Summer Study on High-energy Physics in the 1990s (Snowmass 88), Snowmass, Colorado, 27 Jun - 15 Jul 1988, (1988), p. 613.

127. K. Oide, K. Yokoya: Phys. Rev. A 40 (1989) 315.

128. M. Sullivan, G. Bowden, H. DeStaebler, S. Ecklund, J. Hodgson, T. Mattison, M.E. Nordby, A. Ringwall, et al.: Conf. Proc. C 960610 (1996) 460.

129. B. Aubert, et al. (BABAR Collaboration): Nucl. Instrum. Meth. A 479 (2002) 1.

130. J. Seeman, M. Sullivan, M. Biagini, Y. Cai, F.J. Decker, M. Donald, S. Ecklund, A. Fisher, et al.: Proc. EPAC 2002, 3-7 Jun 2002, Paris, France, (2002), p. 434-436.

131. Y. Yamazaki, T. Kageyama: Part. Accel. 44 (1994) 107.

132. T. Furuya, et al.: Gif-sur-Yvette 1995, RF superconductivity, Vol. 2 (1995), p. 729.

133. H. Schwarz, R. Rimmer: Conf. Proc. C 940627 (1994) 1882.

134. J. Fox, T. Mastorides, C. Rivetta, D. Van Winkle, D. Teytelman: Phys. Rev. ST Accel. Beams 13 (2010) 052802.

135. M. Izawa, Y. Sato, T. Toyomasu: Phys. Rev. Lett. 74 (1995) 5044.

136. K. Ohmi: Phys. Rev. Lett. 75 (1995) 1526.

137. K. Ohmi, F. Zimmermann: Phys. Rev. Lett. 85 (2000) 3821.

138. T.O. Raubenheimer, F. Zimmermann (SLAC): Phys. Rev. E 52 (1995) 5487.

139. J.W. Flanagan, K. Ohmi, H. Fukuma, S. Hiramatsu, M. Tobiyama, E. Perevedentsev: Phys. Rev. Lett. 94 (2005) 054801.

140. H. Fukuma, J. Flanagan, K. Hosoyama, T. Ieiri, T. Kawamoto, T. Kubo, M. Suetake, S. Uno, et al.: AIP Conf. Proc. 642 (2003) 357.

141. Y. Suetsugu, K. Shibata, H. Hisamatsu, M. Shirai, K. Kanazawa: Vacuum 84 (2009) 694.

142. M.T.F. Pivi, F. King, R.E. Kirby, T. Markiewicz, T.O. Raubenheimer, J. Seeman, L. Wang: Conf. Proc. C 0806233 (2008) MOPP064.

143. Y. Suetsugu, H. Fukuma, L. Wang, M. Pivi, A. Morishige, Y. Suzuki, M. Tsukamoto, M. Tsuchiya: Nucl. Instrum. Meth. A 598 (2009) 372.

144. T. Mimashi, T. Ieiri, M. Kikuchi, A. Tokuchi, K. Tsuchida: Conf. Proc. C 0806233 (2008) TUPD011.

145. At least an application for a storage ring is seen in: R. Servranckx, K.L. Brown: IEEE Trans. Nucl. Sci. 26 (1979) 3598.

146. K. Oide, H. Koiso, K. Ohmi: AIP Conf. Proc. 391 (1997) 215.

147. K. Oide, H. Koiso: Phys. Rev. E 47 (1993) 2010.

148. J. Irwin, C.X. Wang, Y.T. Yan, K.L.F. Bane, Y. Cai, F.J. Decker, M.G. Minty, G.V. Stupakov, et al.: Phys. Rev. Lett. 82 (1999) 1684.

149. K. Akai, N. Akasaka, A. Enomoto, J. Flanagan, H. Fukuma, Y. Funakoshi, K. Furukawa, T. Furuya, et al.: Nucl. Instrum. Meth. A 499 (2003) 191. 
150. Y.T. Yan, Y. Cai: Nucl. Instrum. Meth. A 558 (2006) 336.

151. T. Ieiri, K. Akai, H. Fukuma, M. Tobiyama: Nucl. Instrum. Meth. A 606 (2009) 248.

152. K. Satoh, M. Tejima: Conf. Proc. C 950501 (1995) 2482.

153. M. Tejima, M. Arinaga, T. Ieiri, H. Ishii, H. Fukuma, M. Tobiyama, S. Hiramatsu: Conf. Proc. C 0505161 (2005) 3253.

154. A. Drago, J.D. Fox, D. Teytelman, M. Tobiyama: Conf. Proc. C 0806233 (2008) THPC116.

155. T. Mitsuhashi, J.W. Flanagan, S. Hiramatsu: Proc. Seventh EPAC2000, 26-30 Jun 2000, Vienna, Austria, (2000), p. 1783-1785.

156. J.W. Flanagan, N. Akasaka, H. Fukuma, S. Hiramatsu, T. Mitsuhashi, T. Naito, K. Ohmi, K. Oide, et al.: Proc. Seventh EPAC2000, 26-30 Jun 2000, Vienna, Austria, (2000), p. 11191121.

157. N. Akasaka, A. Akiyama, S. Araki, K. Furukawa, T. Katoh, T. Kawamoto, I. Komada, K. Kudo, et al.: Nucl. Instrum. Meth. A 499 (2003) 138.

158. Y. Funakoshi, M. Masuzawa, K. Oide, J. Flanagan, M. Tawada, T. Ieiri, M. Tejima, M. Tobiyama, et al.: Phys. Rev. ST Accel. Beams 10 (2007) 101001.

159. L. Hendrickson, T. Gromme, P. Grossberg, T. Himel, D. Macnair, R. Sass, H. Smith, N. Spencer, et al.: Proc. Seventh EPAC2000, 26-30 Jun 2000, Vienna, Austria, (2000), p. 18971899.

160. K. Ohmi, K. Oide, E. Perevedentsev: Conf. Proc. C 060626 (2006) 616.

161. Y. Ogawa, A. Enomoto, K. Furukawa, T. Kamitani, M. Satoh, T. Sugimura, T. Suwada, Y. Yano, et al.: Conf. Proc. C 060626 (2006) 2700.

162. T. Kamitani, N. Delerue, M. Ikeda, K. Kakihara, S. Ohsawa, T. Oogoe, T. Sugimura, T. Takatomi, et al.: Conf. Proc. C 0505161 (2005) 1233.

163. K. Akai, J. Kirchgessner, D. Moffat, H. Padamsee, J. Sears, T. Stowe, M. Tigner: Proc. B factory workshop, 6-10 Apr 1992, Stanford, California, (1992).

164. K. Hosoyama, K. Akai, K. Ebihara, T. Furuya, K. Hara, T. Honma, A. Kabe, Y. Kojima, et al.: Conf. Proc. C 0806233 (2008) THXM02.

165. K. Ohmi, M. Tawada, Y. Cai, S. Kamada, K. Oide, J. Qiang: Phys. Rev. ST Accel. Beams 7 (2004) 104401.

166. Y. Ohnishi, K. Ohmi, H. Koiso, M. Masuzawa, A. Morita, K. Mori, K. Oide, Y. Seimiya, et al.: Phys. Rev. ST Accel. Beams 12 (2009) 091002.

167. P. Raimondi, D. Shatilov, M. Zobov, "Beam-Beam Issues for Colliding Schemes with Large Piwinski Angle and Crabbed Waist", arXiv:physics/0702033 [physics.acc-ph] (2007).

168. Y. Ohnishi, Proc.15th Annual Meeting of Particle Accelerator Society of Japan August 7-10, 2018, Nagaoka, Niigata, Japan (2018) WEOLP01.

169. SuperKEKB Design Report, https://kds.kek.jp/indico/event/15914/ (2014).

170. Y. Ohnishi, presentation at the 62nd ICFA Advanced Beam Dynamics Workshop on High Luminosity Circular e+e- Colliders (eeFACT2018), September 24-26 (2018), IAS, Hong Kong MOXAA02.

171. K. Ohmi, et al., "Coherent Beam-Beam Instability in Collisions with a Large Crossing Angle", Phys.Rev.Lett. 119 (2017) no.13, 134801.

172. K. Furukawa, et al., Proc. IPAC2018, Vancouver, BC, Canada, doi:10.18429/JACoWIPAC2018-MOPMF073 (2018).

173. Y. Suetsugu, et al., "Mitigating the electron cloud effect in the SuperKEKB positron ring", Phys.Rev.Accel.Beams 22 (2019) no.2, 023201.

174. N. Ohuchi, et al., Proc. IPAC2018, Vancouver, BC, Canada, doi:10.18429/JACoW-IPAC2018TUZGBE2 (2018).

175. A. Accardi et al, Electron Ion Collider: The Next QCD Frontier, arXiv:1212.1701 (2012)

176. https://www.jlab.org/jleic/index.html

177. J Beebe-Wang (Editor), eRHIC, Preconceptual Design Report, arXiv:xxxxxxxx

178. R. Yoshida, EIC Collaboration meeting Oct 2017 (BNL) unpublished, https://indico.bnl.gov/ event/3492/contributions/10260/attachments/9195/11238/yoshida.pdf

179. Yu. Vassiliev et al, Multi-target operation at the HERA-B experiment, AIP Conference Proceedings 512, 359 (2000); https://doi.org/10.1063/1.1291460 
180. R. Brinkmann. Simulation Of Background From Proton Losses In The Hera Straight Sections, Jul 1987. 22 pp. DESY-HERA-87-19 (1987) unpublished

181. http://www.desy.de/mpybar/psdump/eliana-cracow.paper.pdf

182. http://www-hermes.desy.de

183. Ziqing Zhang, Physics from Polarized ep Collisions at HERA, Proceeedings of the 25th Conference of Physics in Collisions, Prague (2005)

184. F. Willeke, Proceedings of the European Particle Accelerator Conference, Lausanne (2004).

185. B. Parker et al, TUOA02A, Proceedings of the European particle Conference 1998, Stockholm(1998); http://accelconf.web.cern.ch/AccelConf/e98/PAPERS/TUOA02A.PDF

186. B. Parker, Serpentine Coil Topology for BNL Direct Wind Superconducting Magnets, Particle Accelerator Conference Knoxville (2005) https://doi.org/10.1109/PAC.2005.1590546

187. N. Ouchi et al, DESIGN OF THE SUPERCONDUCTING MAGNET SYSTEM FOR THE SUPERKEKB INTERACTION REGION, Proceedings of PAC2013, Pasadena, CA USA WEODA1 (2013)

188. B. Parker, M. Anerella, J. Escallier, A. Ghosh, A. Jain, A. Marone, J. Muratore, P. Wanderer, BNL Direct Wind Superconducting Magnets, BNL-96547-2011-CP, Presented at the 22nd International Conference on Magnet Technology (MT-22) Marseille, France September 9-16, (2011) November 2011

189. The LHC Design Report, CERN-2004-003.

190. R. Alemany-Fernandez et al, Operation and Configuration of the LHC in Run 1, CERN-ACCNOTE-2013-0041.

191. J. Wenninger, LHC towards nominal performance, Proceedings of IPAC17, Copenhagen, Dk (2017).

192. Proceedings of the 8th LHC operation workshop, Evian, France (2017), https://indico.cern.ch/ event/663598.

Open Access This chapter is licensed under the terms of the Creative Commons Attribution 4.0 International License (http://creativecommons.org/licenses/by/4.0/), which permits use, sharing, adaptation, distribution and reproduction in any medium or format, as long as you give appropriate credit to the original author(s) and the source, provide a link to the Creative Commons licence and indicate if changes were made.

The images or other third party material in this chapter are included in the chapter's Creative Commons licence, unless indicated otherwise in a credit line to the material. If material is not included in the chapter's Creative Commons licence and your intended use is not permitted by statutory regulation or exceeds the permitted use, you will need to obtain permission directly from the copyright holder. 